Aus der Frauenklinik im Klinikum Kassel

Geschäftsführender Direktor: Prof. Dr. med. Th. Dimpfl des Fachbereichs Medizin der Philipps-Universität Marburg

\title{
Behandlungsergebnisse der exenterativen Eingriffe bei gynäkologischen Malignomen an der Frauenklinik im Klinikum Kassel
}

\author{
Inaugural-Dissertation zur Erlangung \\ des Doktorgrades der gesamten Humanmedizin \\ dem Fachbereich Medizin der Philipps-Universität Marburg \\ vorgelegt von \\ Irina Bogdanova \\ aus Tomsk
}

Marburg, 2008 
Angenommen vom Fachbereich Medizin

der Philipps-Universität Marburg am: 30.07.2008

Gedruckt mit Genehmigung des Fachbereichs

Dekan: Prof. Dr. med. M. Rothmund

Referent: Prof. Dr. med. Th. Dimpfl

Korreferent: PD Dr. med. U. Albert 
Meiner Familie gewidmet 



\section{Inhaltsverzeichnis}

1 Einleitung

1.1. Entwicklung der radikalen onkologischen Chirurgie...........................................4

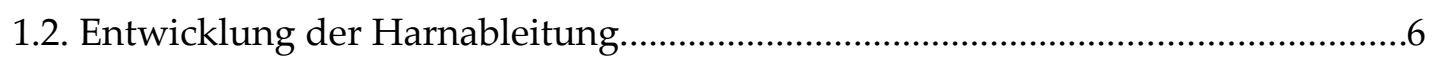

1.3. Entwicklung der Rektumchirurgie...................................................................

1.4. Lebensqualität und Körperleben....................................................................... 8

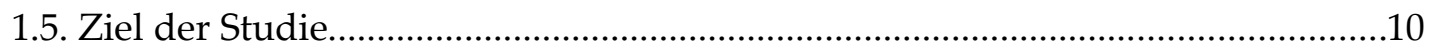

2 Material und Methode

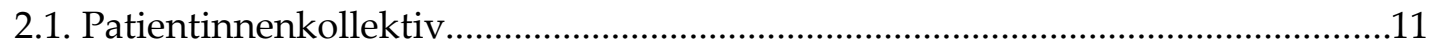

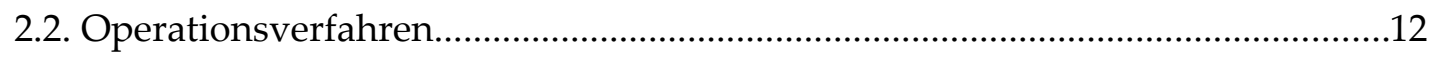

2.3. Rekonstruktionsmöglichkeiten...........................................................................15

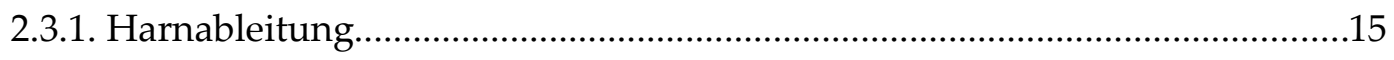

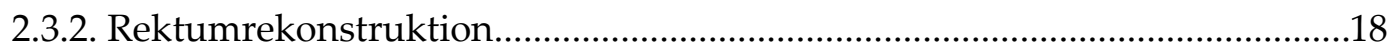

2.3.3. Auskleidung der Wundhöhle im kleinen Becken...........................................19

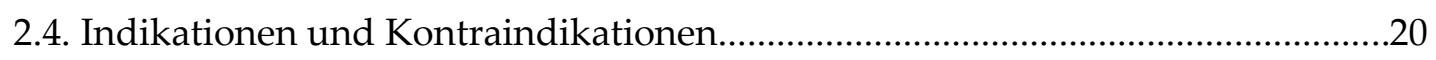

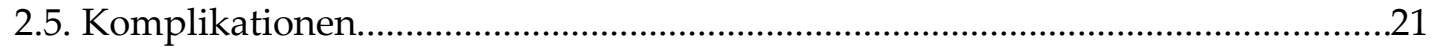

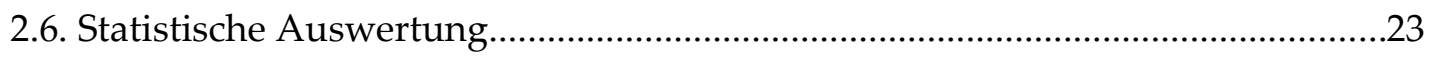

3 Ergebnisse

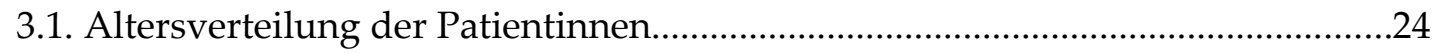

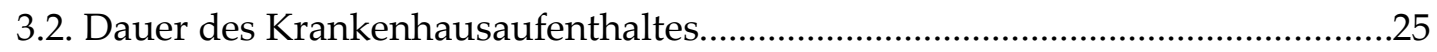

3.3. Operationsindikation und Operationsverfahren.................................................2

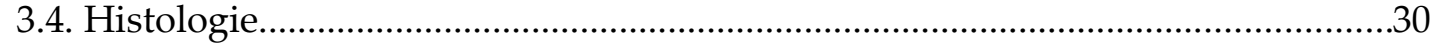

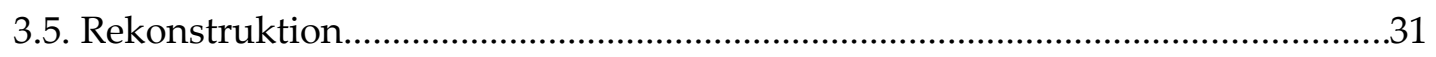

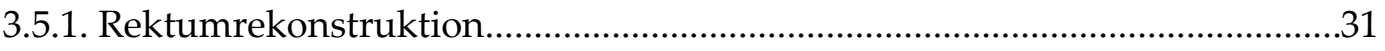

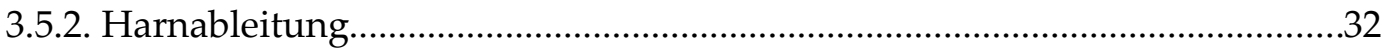

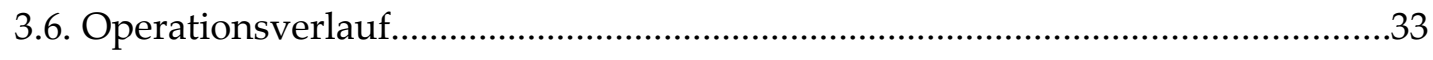




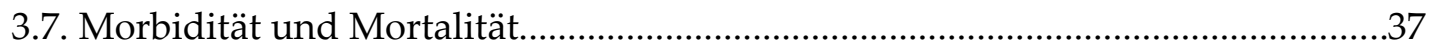

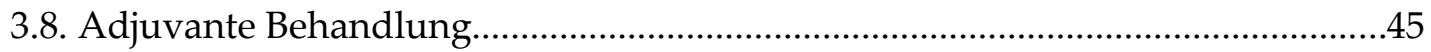

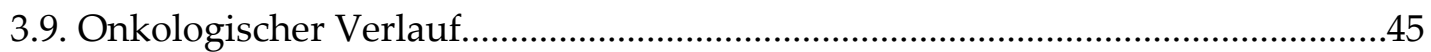

3.10. Einfluss verschiedener Faktoren auf die Gesamtüberlebenszeit........................48

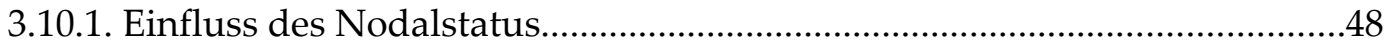

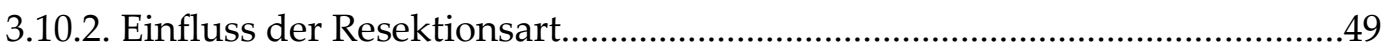

3.10.3. Einfluss der Primär- bzw. Rezidiveingriffe.....................................................50

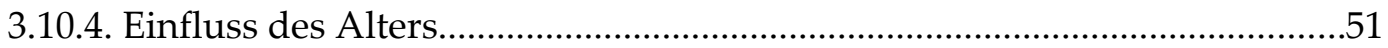

3.10.5. Palliative Exenteration und die Überlebensrate...........................................52

3.11. Einfluss verschiedener Parameter auf die progressionsfreie Überlebenszeit...53

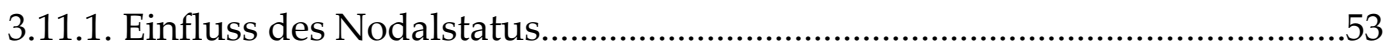

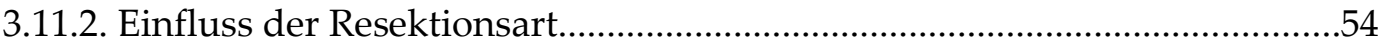

3.11.3. Einfluss der Primär- bzw. Rezidiveingriffe....................................................55

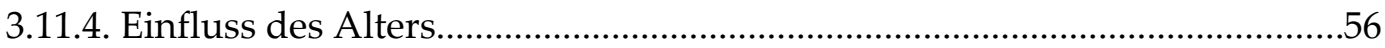

3.12. Überlebenszeit bei verschiedenen Karzinomarten..............................................57

4 Diskussion

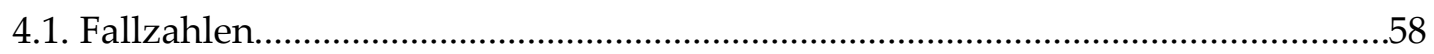

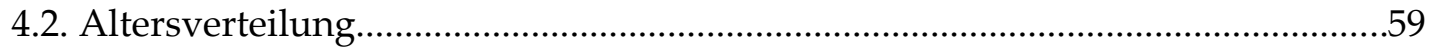

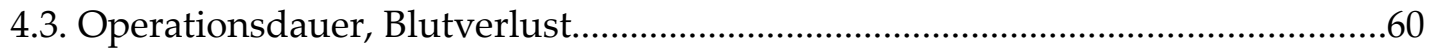

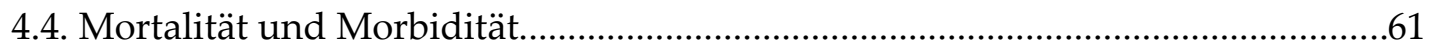

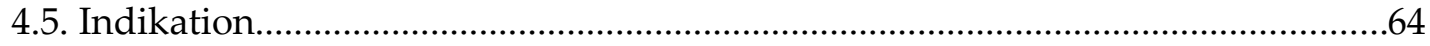

4.6. Gesamt- und progressionsfreie Überlebensraten.................................................66

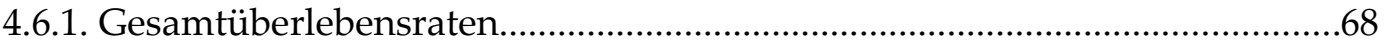

4.6.2. Progressionsfreie Überlebensraten...................................................................71

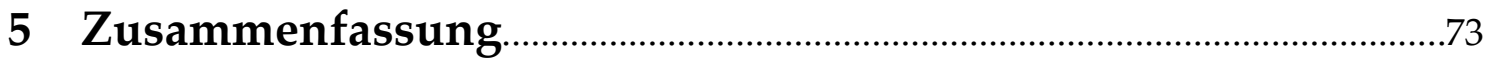

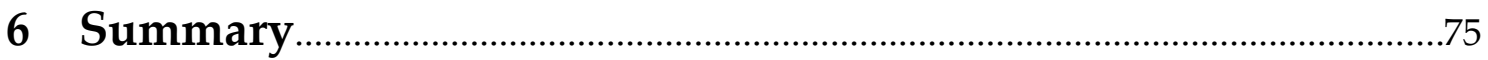




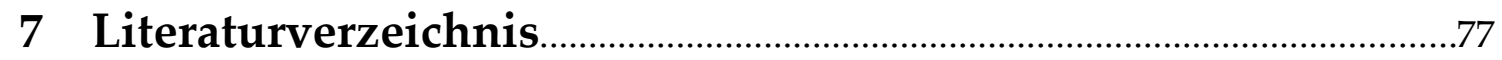

8 Lebenslauf

9 Verzeichnis der akademischen Lehrer................................................91

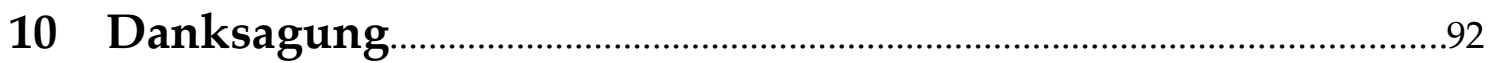

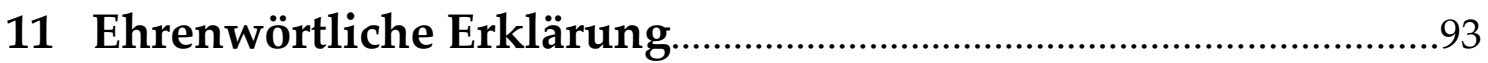




\section{Einleitung}

In der Gynäkologie stellen die organüberschreitenden Tumoren des weiblichen Genitale oder deren (Beckenwand)-Rezidive die Hauptindikation zur Exenteration dar. Bereits 1940 berichtete Bricker über die Exenteration bei fortgeschrittenen Zervixkarzinomen. Eine anfänglich hohe Mortalität und die Beeinträchtigung der Lebensqualität durch zwei inkontinente Stomata waren ausschlaggebend für die überwiegend ablehnende Haltung der Operateure. Die Weiterentwicklung der Chirurgie hat in den letzten Jahrzehnten dazu geführt, dass über die Entfernung erkrankter Organe hinaus ein funktioneller Organersatz möglich ist. Diese Entwicklung hat zu einer enormen und entscheidenden Verbesserung der Lebensqualität für die betroffenen Patientinnen und zur festen Etablierung der Exenteration in der Therapie von Beckentumoren beigetragen.

\subsection{Entwicklung der radikalen onkologischen Chirurgie}

Der Ursprung der radikalen onkologischen Chirurgie geht auf das 19. Jahrhundert zurück. Bereits im Jahre 1809 berichtete Ephraim Mcdowell über die Entfernung eines großen Ovarialtumors bei einer Patientin - seinerzeit noch in deren Haus. Die Weiterentwicklung der radikalen Chirurgie erfolgte in nennenswerter Weise erst in der zweiten Hälfte des 19. Jahrhunderts. Kennzeichnend war, dass die operativen Techniken dieser Zeit - bis auf wenige Ausnahmen - nur auf die Entfernung des befallenen Organs abzielten.

1894 veröffentlichte der New Yorker Chirurg William Halstead die erste Studie über die radikale Mastektomie. Er hatte bei 50 Frauen, bei denen die Erkrankung bereits weit fortgeschritten war, nicht nur die Brustdrüse, sondern auch den darunter liegen- 
den Brustmuskel und die Lymphknoten in der Achselhöhle entfernt. Halstead betonte die immense Bedeutung einer vollständigen Entfernung des befallenen Organs (in seinem Fall der Brust) mit regionaler Lymphonodektomie (Lawrence et al., 1989).

Das Konzept, welches von Halstead ursprünglich für die Mammachirurgie entwickelt wurde, hat die Entwicklung der onkologischen Chirurgie stark beeinflusst und konnte anschließend auch auf andere Bereiche der Chirurgie transferiert werden.

Soweit es um die Entwicklung und Verbesserung der operativen Technik geht, haben sich hierbei insbesondere Billroth (Gastrektomie, Laryngektomie und Prostatektomie), Weir, von Mikulicz und Charles Mayo (Kolonchirurgie), Kocher und Lahey (Schilddrüse-Eingriffe), George Crile und Nicholas Senn, Roswell Park und Hayes Martin (HNOEingriffe), Clark und Wertheim (Eingriffe bei Korpus- und Zervixkarzinomen) verdient gemacht. Während der ersten 40 Jahre des 20. Jahrhunderts wurden neue operative Eingriffe durch Miles (abdominoperineale Resektion, 1908), Graham (Pneumektomie, 1933), Whipple (Pankreatoduodenektomie, 1935) entwickelt.

Nach dem Zweiten Weltkrieg erhöhten sich dank der Fortschritte in der Anästhesie und in der Transfusionsmedizin sowie der Entdeckung der Antibiotika die Einsatzmöglichkeiten der Krebschirurgie.

Eugene M. Bricker, der Pionier der pelvinen Exenteration, vollführte am 8. August 1940 seine erste totale Exenteration an dem neu geschaffenen Ellis Fischel State Cancer Hospital, dem ersten multidisziplinären onkologischen Center westlich des Mississippi. Bricker's Patientin war eine 32-jährige Frau mit einem Zervixkarzinom. Zusammen mit dem Pathologen Lauren Ackerman, dem Radiotherapeuten Theodore Eberhart und Juan del Regato beobachtete Bricker, dass bestimmte, von den Beckenorganen ausgehende Karzinome, die Neigung besitzen, sich für einen langen Zeitraum auf die diesen Bereich zu beschränken, bevor sie außerhalb des Beckens metastasieren (Lopez, Barrios; 2005). 
Bis Mitte des 20. Jahrhunderts hatte sich die Operation, die aus einem Experiment hervorging und kritischen klinischen Situationen vorbehalten war, etabliert. In den Händen einiger weniger Chirurgen bot diese Vorgehensweise eine Möglichkeit zur Heilung für ausgewählte Patienten mit ansonsten unheilbaren und fortgeschrittenen Karzinomen.

A. Brunschwig in New York (1948), L.H. Appleby in Vancouver, BC, Canada (1950) sowie E.S. Britnall und R.H. Flocks in Iowa City (1950) konnten von erfolgreichen Erfahrungen mit dieser Operation berichten.

\subsection{Entwicklung der Harnableitung}

Die erste beschriebene radikale Zystektomie wegen eines Karzinoms stammt von Bardenheuer, der diese am 13.01.1887 im Kölner Bürgerspital bei einer 57-jährigen Frau durchführte. Wegen starker Blutungen wurden die Ureteren in situ belassen und das kleine Becken wurde mit Schwämmen austamponiert. Die Patientin verstarb 14 Tage später am klinischen Bild einer Urämie.

Die Pioniere in der Entwicklung einer Ersatz-Blase waren Tizzoni und Foggi in Bologna, die 1888 einer Hündin ein Ileumsegment auf die Urethra steppten und beide Ureteren in dieses Segment implantierten (Hauri, 2005). Zehn Jahren später konstruierte Gersuny zum ersten Mal beim Menschen eine isolierte Rektalblase und führte sie intrasphinkterisch durch den Analkanal (Hinman, 1936; Hauri, 2005). Dieses Verfahren wurde in der ersten Hälfte des 20. Jahrhunderts populär. In den 40er Jahren arbeitete Bricker an der Entwicklung des Ileum-Conduits, welcher zur Standardmethode der inkontinenten Harnableitung wurde (Bricker, 1950).

Die Geschichte der kontinenten Harnableitung nimmt Anfang des 20. Jahrhunderts ihren Ursprung. 1909 publizierte Verhoogen in Brüssel seine tierexperimentellen Studien, in welchen er die Verwendung eines Ileocoecal-Kompartiments mit der Appen- 
dix als Stoma beschrieb (Hauri, 2005). Gleichzeitig veröffentlichte er seine Erfahrungen bei zwei Patienten nach Zystektomie wegen eines Karzinoms. Ein Jahr später berichtete Makkas aus Bonn über ein Mädchen mit Blasenextrophie (Makkas, 1910). In einer zweizeitigen Operation wurde eine Ersatzblase aus Zoekum mit Appendix als Stoma angelegt. 1950 versuchte Gilchrist in Chicago, die Kontinenz mittels Ileocoecalklappe zu erreichen (Gilchrist et al., 1950).

Die 80er Jahre des 20. Jahrhunderts sind durch große Fortschritte bei der Entwicklung von Harnableitungen geprägt. Dies liegt zum einem an der weiten Verbreitung und Verbesserung der Darmersatzblasen, zum anderen an der Entwicklung einer Anzahl von Darm-Pouches mit kontinentem Stoma. Kock schuf den Kock-Pouch 1982, Thüroff den Mainz-Pouch I 1985, Rowland 1987 den Indiana-Pouch (Hauri, 2005).

\subsection{Entwicklung der Rektumchirurgie}

Erste chirurgische Methoden der Rektumresektion gingen vom Perineum aus und wurden von Faget (1739), Lisfranc (1826) und Verneuil (1837) durchgeführt. Die verwendete Operationstechnik vollzog sich ausschließlich extraperitoneal, was im Zeitalter der fehlenden Asepsis und der fehlenden Antibiotika von entscheidender Bedeutung war. Trotzdem überlebten nur die wenigsten Patienten diese Operationen. Entscheidende Fortschritte wurden durch Allingham (1879), der zur Vermeidung einer Sepsis das Vorschalten eines Kolostomas propagierte, sowie durch Lockhart-Mummery (1926), der neben dem Kolostoma großen Wert auf die Grundzüge des aseptischen Operierens legte, erzielt. Hochenegg entwickelte 1888 als Erster die so genannte Durchzugmethode, bei der nach der Resektion des Rektums das proximale Kolon mobilisiert, durch den erhaltenen Analkanal ausgeführt und an dessen Außenrand fixiert wurde. Aufgrund der von Miles aufgestellten These, dass eine kurative Behandlung eines Rektumkarzinoms, unabhängig von seiner Lage, lediglich durch eine radi- 
kale abdominoperineale Rektumamputation erfolgen könne, wurde zu dieser Zeit von den sphinktererhaltenden Methoden abgesehen. Erst die Ergebnisse von Dukes (1930), Westhues (1930), Gilchrist und David (1938), die aufzeigten, dass Miles Erkenntnisse nicht uneingeschränkt richtig waren, gaben der Rektumchirurgie eine entscheidende neue Richtung und führten zu einer Vielzahl neuer sowie zur Wiederaufnahme älterer sphinktererhaltender Operationstechniken. Zu dieser Zeit ging man jedoch immer noch davon aus, dass zur Kontinenzerhaltung stets ein Rektumstumpf von mindestens 6-8 cm erhalten werden müsse. Erst 1972 ergaben sich wieder entscheidende Neuerungen durch die von Parks beschriebene abdominotransanale Durchzugmethode, bei der die Anastomose zwischen Kolon und Anorektum durch einen dilatierten Analkanal genäht wurde. Damit konnte erstmals gezeigt werden, dass auch nach Extirpation des gesamten Mastdarms eine ausreichende Kontinenz erreicht werden kann.

Einen weiteren wichtigen Schritt stellte 1975 die Einführung der russischen Klammernahtgeräte durch Fain dar. Durch die Weiterentwicklung des in vielen Bereichen verbesserten amerikanischen Staplers etablierte sich die Stapling-Technik endgültig in der Rektumchirurgie.

\subsection{Lebensqualität und Körperleben}

Bereits 1950 hielt Bricker fest, „dass keine moralische Verpflichtung zur Durchführung der Operation besteht, wenn wir dadurch den Patienten nicht in einen funktionalen Zustand versetzen können, der ihm eine komfortable Existenz ermöglicht" (Bricker, 1950). Die Verbesserung der onkologischen Behandlung und Patientenselektion sowie eine verlängerte Lebenserwartung haben dazu führt, dass heutzutage die Lebensqualität nach radikaler Beckenchirurgie bei häufig jungen Patientinnen immer mehr an Bedeutung gewinnt und im Mittelpunkt verschiedener Studien steht. 
In einer Untersuchung wurde das körperlich-sexuelle Empfinden von Patientinnen vor und nach gynäkologischen Operationen (Hysterektomie, Wertheim'sche Radikaloperation, Exenteration) untersucht. Während Frauen nach Hysterektomie ein normales Körpererleben angegeben haben, fühlten sich Karzinompatientinnen in Abhängigkeit therapiebedingter körperlicher Veränderungen weniger attraktiv und in ihrem Selbstwertgefühl eingeschränkter. Bei den Exenterationspatientinnen waren postoperativ lediglich 29\% mit ihrem körperlich-sexuellen Erleben zufrieden (Schönefuß et al., 2001). B.L. Andersen hat gezeigt, dass die Exenteration bei vielen Patientinnen zur totalen Einstellung der sexueller Aktivitäten führt: 57\% der betroffenen Frauen fühlten sich ein Jahr nach der Operation immer noch in ihrer Sexualität „wie blockiert“, vor allem dann, wenn Stomata angelegt werden mussten (Andersen, 1985). Hawighorst et al haben gezeigt, dass postoperativ die Gesamtbelastung der Lebensqualität bei Patientinnen mit zwei Stomata im Vergleich zu rekonstruktiv behandelten Patientinnen deutlich ansteigt (Hawighorst et al., 2004). Ein ähnliches Bild zeigt sich bei Patientinnen, deren vaginale Kapazität nicht erhalten bzw. nicht wiederhergestellt werden konnte. Postoperativ geben diese Patientinnen eine höhere Beeinträchtigung ihrer Lebensqualität an, als Patientinnen mit Neovagina oder (teil-) erhaltener Vaginalkapazität.

Allein die Möglichkeit zur vaginalen Kohabitation, selbst durch eine Ersatzvagina, erhöht nach Ansicht der Autoren die Lebensqualität der Patientinnen. In diesem Sinne wirken sich auch die kontinenten Stomata und die End-zu-End-Anastomosen in allen Bereichen der Lebensqualität aus (Hawighorst et al., 2004; Schönefuß et al., 2001).

Nicht nur eine operative Therapie sondern auch eine adjuvante Behandlung kann die Lebensqualität beeinflussen. Hawighorst hat in seiner Studie gezeigt, dass Frauen, die sich im Anschluss an die Operation einer adjuvanten Therapie unterziehen müssen, mehr körperliche und sexuelle Probleme sowie eine geringere Lebensqualität angeben als die Frauen, denen postoperativ keine adjuvante Therapie bevorsteht (Hawighorst et al., 2004). B.L. Andersen konnte bei 6 bis 19\% der operierten und bei 44 bis 79\% der bestrahlten Patientinnen nachhaltige Störungen im Sexualverhalten beobachten (An- 
dersen, 1985). In einem von M. Tamburini et al. untersuchten Kollektiv von Zervixkarzinom-Patientinnen hatten $36 \%$ der operierten und $46 \%$ der hysterektomierten und auch bestrahlten Frauen sexuelle Probleme (Tamburini et al., 1986).

\subsection{Ziel der Studie}

In dieser Arbeit werden die Behandlungsergebnisse von 66 an der Frauenklinik im Klinikum Kassel zwischen 1991 und 2005 operierten Patientinnen mit gynäkologischen Malignomen erfasst. Im Ergebnisteil werden Daten zum Patientinnenkollektiv, zur Überlebenswahrscheinlichkeit und zur Häufigkeit von Rezidiven analysiert und im nationalen und internationalen Vergleich eingeordnet. Zusätzlich werden klinische und histopathologische Parameter hinsichtlich der Gesamtüberlebenszeit und der progressionsfreien Überlebenszeit untersucht. Als progressionsfreie Überlebenszeit wurde die Zeit vom chirurgischen Eingriff bis zum Auftreten entweder eines lokoregionären Rezidivs oder von Fernmetastasen definiert. 


\section{Material und Methode}

\subsection{Patientinnenkollektiv}

In die vorliegende retrospektive Studie wurden alle Patientinnen aufgenommen, die im Zeitraum von 01.01.1991 bis 31.12.2005 an der Frauenklinik im Klinikum Kassel exenteriert wurden. Die Datenerfassung erfolgte durch Einsicht in die Operationsbücher und Akten aus dem Archiv des Klinikums Kassel.

Der weitere Krankheitsverlauf, Nachbehandlungen sowie das Befinden der Patientinnen wurden anhand der vorliegenden Unterlagen der klinikinternen Nachsorgen erfasst. Wenn die Nachsorge nicht in der Klinik durchgeführt war, wurden die erforderlichen Daten durch Befragung der behandelnden Hausärzte oder Frauenärzte erhoben. Das Datum 30.09.2006 war der letzte Einschlusstag für die Bewertung des postoperativen Verlaufs.

Bei vier Patientinnen konnte kein weiterer Verlauf nachvollzogen werden. Diese Patientinnen waren weder bei dem angegeben Gynäkologen oder Hausarzt weiter in Betreuung, noch über die angegebene Adresse erreichbar.

Zur Erstellung der Datenbasis wurden eine Reihe Parameter definiert, nach denen die Krankenakten analysiert wurden. Die auf diese Weise gewonnenen Daten wurden in einer Tabelle erfasst. 
Folgende Parameter wurden erfasst:

- Stammdaten (der Patientinnenname, das Geburtsdatum)

- Anamnestische Daten (bisheriger Krankheitsverlauf, Vorbehandlungen)

- Daten zur Operation (Datum, Art der Exenteration, OP-Dauer, Transfusionsmenge, Art der Harn- und Stuhlableitung)

- Histologischer Befund (Tumorart, Lymphknotenbefall, Resektion in sano/non in sano)

- Postoperativer Krankheitsverlauf (postoperative Komplikationen, Therapie)

- Adjuvante Therapie

- Onkologischer Verlauf

- Lokalrezidiv (Diagnosedatum, postoperative rezidivfreie Überlebenszeit)

- Fernmetastasen (Diagnosedatum, postoperative metastasenfreie Überlebenszeit)

- Tod (Todesdatum, postoperative Gesamtüberlebenszeit)

\subsection{Operationsverfahren}

Bei allen Patientinnen wurden die Exenterationsart sowie die Art der Rekonstruktion erfasst. Zusätzlich wurden die Dauer des Krankenhausaufenthaltes, die Dauer der Operation und die benötigte Anzahl der Erythrozytenkonzentrate während der Operation berücksichtigt. Diese Parameter wurden in verschiedenen Patientinnengruppen verglichen (Altersgruppe, Primär- vs. Rezidiveingriff, vorangegangene Radiatio vs. keine Radiatio). 
Das Ausmaß der Exenteration wird unterteilt in:

- vordere Exenteration: partielle oder radikale Kolpektomie, radikale Hysterektomie (falls vorhanden), Entfernung der Harnblase

- hintere Exenteration: partielle oder radikale Kolpektomie, radikale Hysterektomie (falls vorhanden), partielle oder vollständige Entfernung des Rektosigmoids

- komplette Exenteration: vordere und hintere Exenteration

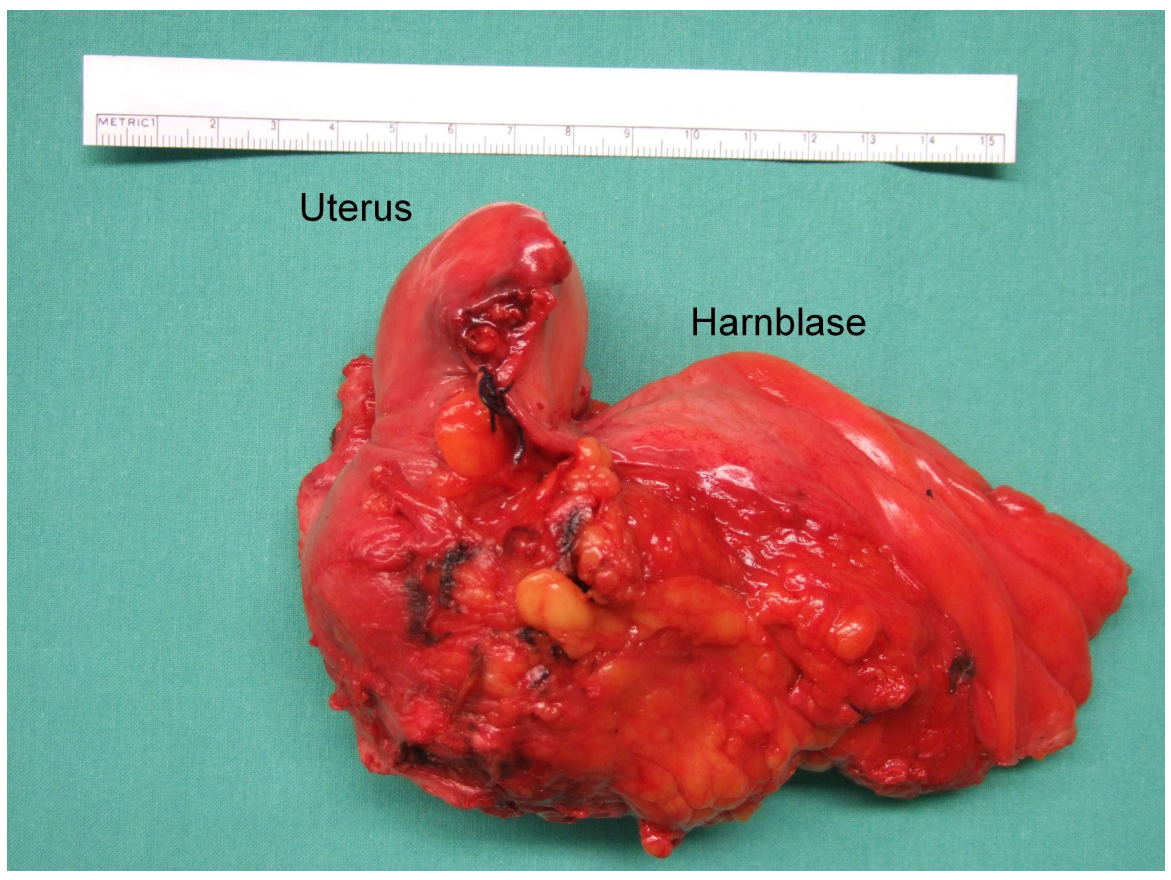

Abbildung 1. Exenterationspräparat nach einer vorderen Exenteration wegen eines fortgeschrittenen Zervixkarzinoms 


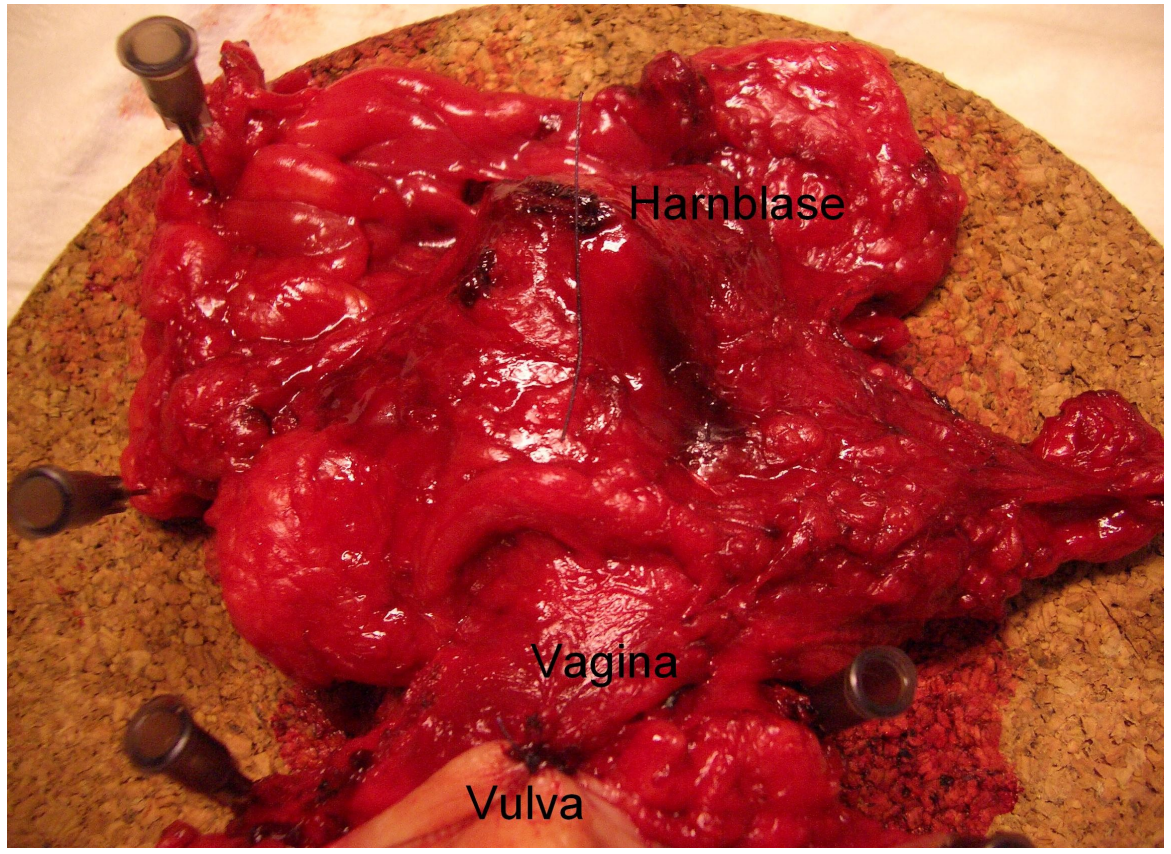

Abbildung 2. Exenterationspräparat nach einer vorderen Exenteration mit totaler

Kolpektomie und Vulvektomie bei einem Rezidiv eines Vaginalkarzinoms

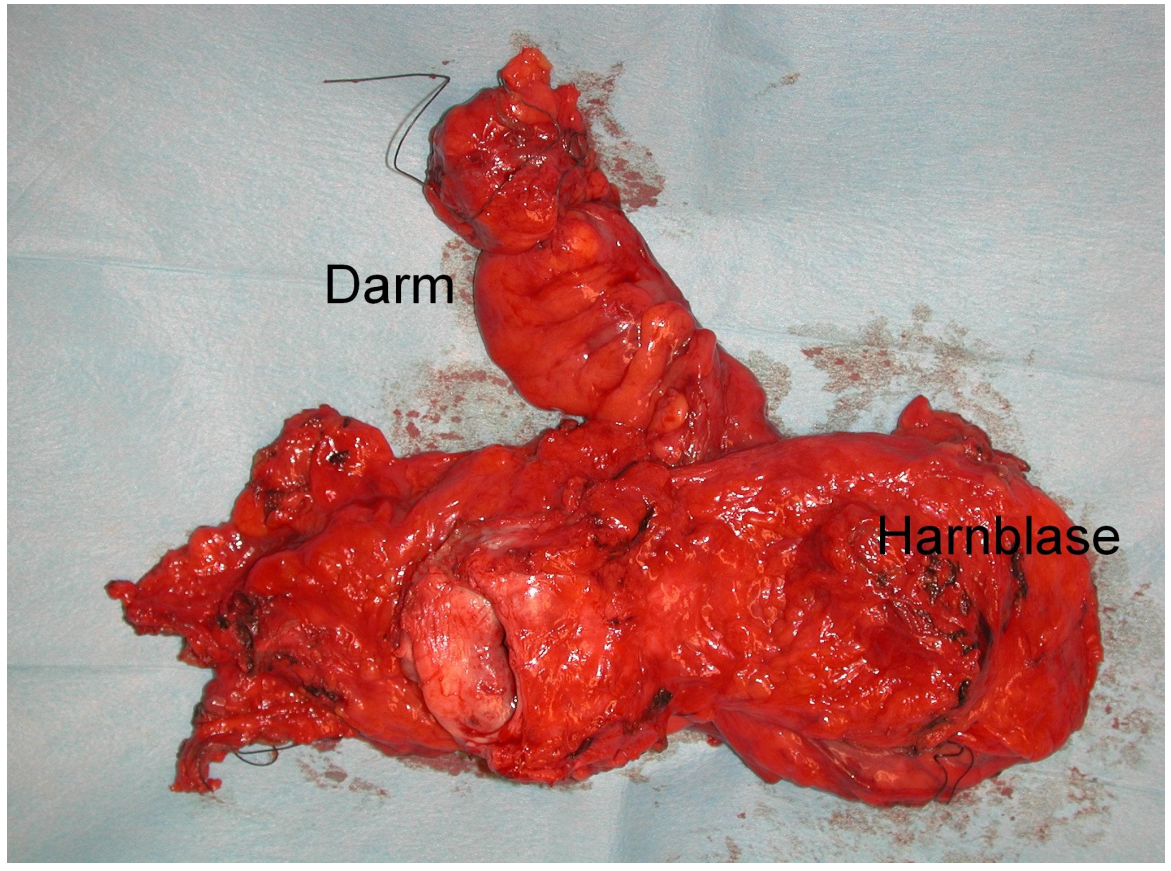

Abbildung 3. Exenterationspräparat nach einer totalen Exenteration wegen eines Zervixkarzinomrezidivs 
Daneben wird in der Literatur zwischen drei verschiedenen Exenterationstypen in $\mathrm{Ab}$ hängigkeit von der Beckenanatomie unterschieden (Hillemans et al., 2004; Magrina et al., 1997):

- $\quad$ supralevatorische Exenteration (Typ I): Exenteration der Beckenorgane oberhalb der Levatorplatte

- translevatorische Exenteration (Typ II): partielle Resektion von Muskulus levator und Diaphragma urogenitale

- translevatorische Exenteration mit Vulvektomie (Typ III): Vulvektomie, radikale Resektion von Muskulus levator, Diaphragma urogenitale und vulvo-perineales Weichgewebe.

\subsection{Rekonstruktionsmöglichkeiten}

Bei allen Patientinnen wurde die Art der Rekonstruktion nach Exenteration erfasst.

\subsubsection{Harnableitung}

Nach der Entfernung der Blase stehen prinzipiell zwei Formen der Harnableitung zur Verfügung. Bei den inkontinenten Formen wird im Wesentlichen das Ileum oder Kolon-Conduit verwendet. Die früher häufig durchgeführte beidseitige Ureterokutaneostomie (zwei inkontinente Stomata) oder Transureterokutaneostomie (ein inkontinentes Stoma) werden aufgrund der hohen Komplikationsrate (Abknicken des Ureters und Stomastenosen) nur sehr selten angewendet (Hirsch, 1994). Diese Verfahren sind die Ultima Ratio, wenn von Seiten der Patientin entsprechender Leidensdruck besteht, eine Harnableitung erforderlich ist und eine andere operative Technik nicht zur Anwendung kommen kann (Lampe et al., 2005). 
Die kontinenten Formen werden in drei Gruppen unterteilt: die Ersatzblasen, gebildet aus Ileum und/oder Kolon, die rektalen Reservoire (Ureterosigmoidostomie oder Sigma-Rektum-Pouch) und die Blasensubstitution mittels interstinaler Segmente (vom Magen bis hin zum Sigma) (Hirsch, 1994; Hohenfellner, 1994).

Die von E.M. Bricker eingeführte Zwischenschaltung eines Dünndarmsegmentes zur Harnableitung ist heutzutage immer noch die am weitesten verbreitete Harnableitungsform nach Zystektomie (Novak, 2002; Egger, 1999; Hautmann, 2007; Gillitzer, 2007). Die relative kurze Operationszeit, die geringe Länge des benötigten Darms (12 bis $15 \mathrm{~cm}$ terminales Ileum) und die geringe perioperative Komplikationsrate lassen das Ileum-Conduit bei älteren Patientinnen und solchen mit einem hohen operativen Risiko sowie in der palliativen Situation (Vermeidung einer Kloakenbildung) als Harnableitung der Wahl erscheinen (Miller, 2003). Zudem besteht keine Gefahr der Ausbildung eines Kurzdarmsyndroms, im Gegensatz zur kontinenten Harnableitung, die wesentlich längere Darmsegmente $(60-80 \mathrm{~cm})$ benötigt (Gillitzer, 2007). Die Ureteren werden dabei in das orale Ende des ausgeschalteten Ileumsegments implantiert, das aborale Ende wird als Stoma im Normfall in den rechten Unterbauch platziert. Die Frühkomplikationsrate (Harntransportstörung, Rektumscheidenfistel, Platzbauch) liegt bei 24-56\% (Morgan et al., 1993; Stein, 1996). Spätkomplikationen können bei zwei Drittel der Patientinnen nach Ileum-Conduit auftreten (Madersbacher, 2003). Dies betrifft insbesondere Komplikationen des Stomas beim Ileum-Conduit. In den Studien von Madersbacher und Iborra wird die Stomastenose mit 11\% als die häufigste revisionsbedürftige conduitspezifische Spätkomplikation angegeben, die jedoch in bis zu 78\% der Fälle innerhalb von fünf Jahren nach der Operation auftritt (Madersbacher, 2003; Iborra, 2001). Ein operationsbedürftiger Darmverschluss und/oder eine Fistelbildung kommen ebenfalls in $11 \%$ der Fälle vor, Komplikationen der Ureterdarmanastomose in bis zu etwa $14 \%$. Hospitalisationsbedürftige Harnwegsinfekte und Pyelonephritiden werden mit einer Häufigkeit von bis zu 23\% angegeben. Im Langzeitverlauf von über 10 Jahren entwickeln 24-38\% der Fälle eine Urolithiasis. (Lampe et al., 2005; Rauchen- 
wald, 2001; Iborra, 2001; Madersbacher, 2003; Gillitzer, 2007). Anders als bei kontinenten Harnableitungen ist die Ausbildung einer substitutionspflichtigen hypochlorämischen Azidose seltener. Dies erklärt sich vor allem durch den kurzzeitigen Urinkontakt mit der Schleimhaut und durch die geringe Resorptionsfläche des Darminterponates (Gillitzer, 2007).

Das 1952 von Übelhör eingeführte, operativ aufwendigere antirefluxive Kolon-Conduit hat sich bezüglich der Spätkomplikationen als vorteilhaft erwiesen (Übelhör, 1952; Stein, 1996).

Kann das Sigma nicht verwendet werden (Strahlenschaden, Divertikulitis) oder ist es zu kurz, um eine spannungsfreie Anastomose zu gewährleisten, so stellt das Transversum-Conduit eine gute Alternative dar.

Die wesentlich fundierten Kenntnisse der autonomen und somatischen Innervation des Sphinkterapparates sowie wichtige chirurgische Neuerungen haben in den letzten zwei Jahrzehnten die Verbreitung verschiedener Formen der kontinenten Harnableitungen ermöglicht. Die kombinierte Verwendung von Dünndarm und Dickdarm hat sich in den meisten Zentren durchgesetzt, da durch dieses Vorgehen eine antirefluxive Ureterimplantation, ein gutes initiales Volumen und ein effizienter efferenter Verschlussmechanismus erreicht werden können. Je nach Modifikation werden $30 \mathrm{~cm}$ terminales Ileum und $15 \mathrm{~cm}$ Zökum und Colon ascendens ausgeschaltet. Als efferenter Anti-Refluxmechanismus dient entweder die Appendix oder ein rekonfiguriertes $5 \mathrm{~cm}$ langes Ileumsegment. Als externes Stoma bietet sich zumeist der Nabel an (Hautmann et al., 1999; Rowland, 1996; Stein, 1996; Stief, 2002).

Durch die kontinenten Formen der Harnableitung, welche ein äußerlich sichtbares Stoma und das Tragen eines Stomabeutels vermeiden, hat sich auch die Lebensqualität der Patienten und ihre soziale Integration deutlich gebessert (Boyd et al., 1987; Hawighorst, 2004). 
Absolute Kontraindikation für eine kontinente Harnableitung sind eine deutlich eingeschränkte Nierenfunktion mit Serum-Kreatinin-Werten > 2-2,5 mg/dl, eine schwere Leberinsuffizienz oder entzündliche Darmerkrankung. Da jede Patientin nach kontinenter Harnableitung zum intermittierenden Selbstkatheterismus fähig sein muss, sollen Alter, kognitive Fähigkeiten und Fingerfertigkeit stets berücksichtigt werden. Zudem können weitere Erkrankungen, z.B. Morbus Parkinson oder multiple Sklerose, die Fähigkeit zum Selbstkatheterismus entscheidend limitieren. (Rauchenwald, 2001; Gillitzer, 2007).

\subsubsection{Rektumrekonstruktion}

Fortgeschrittene Karzinome im kleinen Becken betreffen häufig auch den Darm. In Abhängigkeit von der Höhe der Resektion kann der Sphinkterapparat erhalten werden. Um die Darmkontinuität zu erhalten, können maximal obere Anteile des inneren Sphinkters reseziert werden, eine so genannte intersphinktäre Resektion in Höhe der Linea dentata. Die Rekonstruktion kann dann als gerade koloanale Anastomose erfolgen (Lampe et al., 2005).

Die so versorgten Patienten leiden jedoch meist unter Einschränkungen der differenzierten Kontinenzleistungen, Stuhlfrequenz und Stuhldrang sind vor allem in den ersten 2 Jahren postoperativ beeinträchtigt. Dies kann zu einem guten Teil vermieden werden durch die Bildung eines Kolon-Pouches als Neoreservoir (Hallböök et al., 1996; Lazorthes et al., 1997; Willis et al., 2001).

Die Formung eines J-förmigen Pouches aus dem Colon descensus wird heute in vielen Kliniken regelmäßig nach tiefer Rektumresektion durchgeführt. Dazu wird die Kolonschlinge J-förmig aneinander gelegt und Seit-zu-Seit anastomosiert. Die pouchanale Anastomose erfolgt in der Regel mit dem Stapler Seit-zu-End (Lampe et al., 2005).

Der Kolon-Pouch sollte eine Länge von 5 bis $6 \mathrm{~cm}$ nicht überschreiten, um Entlee- 
rungsprobleme zu vermeiden. Neben der verbesserten Kontinenzsituation füllt der Kolon-Pouch das Becken sehr viel besser aus als das gerade hinunter geführte Kolon. Dies vermeidet das Hinuntergleiten von Dünndarmanteilen, die bei späterer Bestrahlung oder bei einem Rezidiv erheblich stärker gefährdet sind als das Kolon.

Alternativ zum Kolon-J-Pouch wurden auch andere Verfahren zur Bildung eines NeoReservoirs beschrieben. Unter diesen haben der Zökum-Pouch und die Koloplastik eine gewisse Bedeutung erlangt. Erste Erfahrungen an kleinen Patientenkollektiven zeigen gute funktionelle Ergebnisse bezüglich Stuhlfrequenz und -drang. Etwas häufiger traten jedoch Anastomoseinsuffizienzen auf, unter Umständen bedingt durch die beeinträchtigte Vaskularisation der End-zu-End Anastomose (Mantyh et al., 2001; Lampe et al., 2005).

\subsubsection{Auskleidung der Wundhöhle im kleinen Becken}

In den letzten Jahren haben sich zwei Verfahren bewährt, nämlich die Auskleidung der Beckenhöhle mit einem gestielten Netzlappen oder mit einem freien bzw. gestielten Lappen aus Peritoneum. Der Netzlappen bietet den Vorteil intakter Gefäßversorgung, was insbesondere nach Bestrahlung von Bedeutung sein kann. Die Auskleidung mit Peritoneum ist einfacher und geht schneller, entsprechendes Gewebe steht immer zur Verfügung.

Die Auskleidung des kleinen Beckens mit Amnion, tierischem Gewebe, Dura mater und mobilisiertem Sigma oder Zökum, die Verwendung synthetischer Materialien und Beckentamponaden allein, wie von Brunschwig beschrieben, haben sich nicht durchgesetzt (Hirsch et al., 1994). 


\subsection{Indikationen und Kontraindikationen}

Auf Grund der Intensität dieses Eingriffs wird die pelvine Exenteration bis in die heutige Zeit nur zögerlich ausgeführt und potenzielle Patienten werden einer kritischen Auswahl unterzogen.

Prinzipiell erscheinen Tumore, die sich durch ein vorzugsweise lokales Wachstum und durch eine verzögerte Fernmetastasierung auszeichnen, für eine Exenteration geeignet, was ebenso für Rezidive gilt.

Die häufigste Indikation für eine pelvine Exenteration stellt ein fortgeschrittenes Zervixkarzinom oder ein Zentralrezidiv eines Zervixkarzinoms dar. Das zentrale Rezidiv wird definiert durch einen Tumor im Bereich von Zervix oder Vagina im Falle der primären Radiatio/Radiochemotherapie bzw. des Scheidenabschlusses und Spatium recto-vaginale im Zustand nach chirurgischer Therapie (Hillemanns et al., 2004).

Andere Indikationen sind Rezidive oder erfolglos behandelte Malignome der Beckenorgane wie Karzinome oder Sarkome des Corpus uteri, der Vagina, des Rektums, der Blase oder der Vulva.

Bei Patientinnen mit Ovarialkarzinomen ist die Voraussetzung für eine Exenterationsoperation wegen der frühzeitigen intraperitonealen Metastasierung selten gegeben (Petri, 2001; Lopez et al., 2005; Aleksic, 1998; Rodrigues-Bigas et al., 1996; Lambrou et al., 2005). Unter bestimmten Voraussetzungen können jedoch die ausgedehnten abdominalchirurgischen Eingriffe sowohl bei einer Primäroperation als auch beim Auftreten eines späten platinsensiblen Rezidivs eines Ovarialkarzinoms die Rate der postoperativen Tumorfreiheit und somit die Prognose der Patientinnen verbessern. Als prognostisch günstige Parameter für einen Operationserfolg und damit für das Überleben werden ein guter Allgemeinzustand, Tumorfreiheit nach einer Primäroperation oder niedriges Tumorstadium bei Erstdiagnose sowie kein Nachweis von Aszites beim Rezidiv genannt (Schröer et al., 2006; Buttarelli et al., 2006, Harter et al., 2006). 
Die Kontraindikationen können allgemeiner und lokaler Natur sein. Allgemeine Gründe gegen derartige Operationen sind: reduzierter Allgemeinzustand, höheres Alter, psychische und physische Anpassungsschwierigkeiten gegenüber derartigen Eingriffen und ihren Folgen, erheblicher Nierenschaden mit beginnender Urämie und vor allem die extrapelvine Metastasierung (Hirsch et al., 1994). Eine solche muss präoperativ ausgeschlossen werden (Thoraxaufnahme, Lebersonographie, CT/MRT, unter Umständen Skalenusbiopsie oder intraoperative Biopsie paraaortaler Lymphknoten). Als lokale Kontraindikation einer Beckenexenteration sind zu nennen: auf das Beckenskelett übergreifende Karzinominfiltrationen, Tumorinfiltrationen im Bereich der Spina ischiadica mit Ischialgien oder Beinödemen (Lopez et al., 2005; Rodrigues-Bigas et al., 1996; Lambrou et al., 2005).

\subsection{Komplikationen}

Bei allen Patientinnen wurden postoperative Komplikationen, die bei dem Krankenhausaufenthalt aufgetreten sind, erfasst. Dabei wurden folgende chirurgische Komplikationen registriert:

- Peritonitis

- Blutung

- Wundheilungsstörung

- Anastomoseinsuffizienz

- Ileus und Subileus

- Abszess 
Als postoperative nicht-chirurgische Komplikationen wurden folgende Komplikationen erfasst:

- kardial

- pulmonal

- neurologisch

- renal

Zusätzlich wurde der postoperative Verlauf je nach Schweregrad der aufgetretenen Komplikationen in folgenden Gruppen eingeteilt:

1. unauffälliger postoperativer Verlauf

2. leichte Komplikationen

3. mäßige Komplikationen

4. ausgeprägte Komplikationen

5. lebensbedrohliche Komplikationen

6. tödliche Komplikationen

Als leichte Komplikationen galten die Komplikationen, die spontan und ohne spezielle therapeutische Maßnahmen abgeklungen sind (z.B. Appetitlosigkeit, depressive Verstimmung, reversible Harnstau, etc). Die mäßigen Komplikationen waren mit einfachen Maßnahmen zu behandeln und die Dauer des Krankenhausaufenthaltes war dadurch nicht wesentlich verlängert (z.B. oberflächliche Wundheilungsstörung, unkomplizierter Harnwegsinfekt, Thrombophlebitis, etc). Komplikationen, die eine Einleitung von intensiven medikamentösen und supportiven Maßnahmen erforderten (z.B. intravenöse Gabe von Antibiotika, zentral wirkende Analgetika, antikoagulative Therapie, chirurgische Intervention) galten als ausgeprägt. Die lebensbedrohlichen Komplikationen erforderten eine umgehende Einleitung intensiv-medizinischer Maßnahmen oder ausgedehnter chirurgischer Interventionen (z.B. Anastomoseinsuffizienz, Peritonitis, Ileus, Thromboembolie, etc). Organspezifische Nebenwirkungen, die zum Tode führen, wurden als tödliche Komplikationen definiert. 
Als Grundlage für diese Klassifikation wurden die vom National Cancer Institute entwickelten Kriterien zur Erfassung von Nebenwirkungen nach Chemo- oder Radiotherapie benutzt (Common Toxicity Criteria (CTC)), da keine einheitliche Klassifikation der postoperativen Komplikationen in der Literatur gefunden werden konnte.

Die Häufigkeit postoperativer Komplikationen je nach Schweregrad wurde in verschiedenen Patientinnengruppen verglichen (Altersgruppe, Primär- vs. Rezidiveingriff, vorangegangene Radiatio vs. keine Radiatio).

Es wurde eine intra- und postoperative Mortalität erfasst.

\subsection{Statistische Auswertung}

Die Datenerfassung und Auswertung erfolgten mit dem Programm SPSS in der Version 13.0.

Es wurde zunächst die deskriptive statistische Beschreibung der einzelnen Parameter durchgeführt. Der Vergleich relativer Häufigkeit der Merkmale erfolgte mit Hilfe des $\chi^{2}$ - Tests. Damit wurde geprüft, ob ein statistisch signifikanter Zusammenhang besteht. Weiterhin wurden Variablen definiert und ihr Einfluss auf die progressionsfreie Überlebenszeit und tumorbezogene Gesamtüberlebenszeit nach der Methode von KaplanMeier untersucht. Die Überprüfung der statistischen Signifikanz erfolgte mit dem LogRank Test.

Das Signifikanzniveau wurde bei 5\% Irrtumswahrscheinlichkeit definiert. 


\section{Ergebnisse}

\subsection{Altersverteilung der Patientinnen}

Das Alter der Patientinnen wurde zum Zeitpunkt der Operation erhoben.

Der Altersdurchschnitt betrug 59,1 Jahre, wobei die jüngste Patientin 34 Jahre und die älteste Patientin 87 Jahre alt waren. Der Median betrug 60 Jahre, die Standardabweichung 13,9 Jahre.

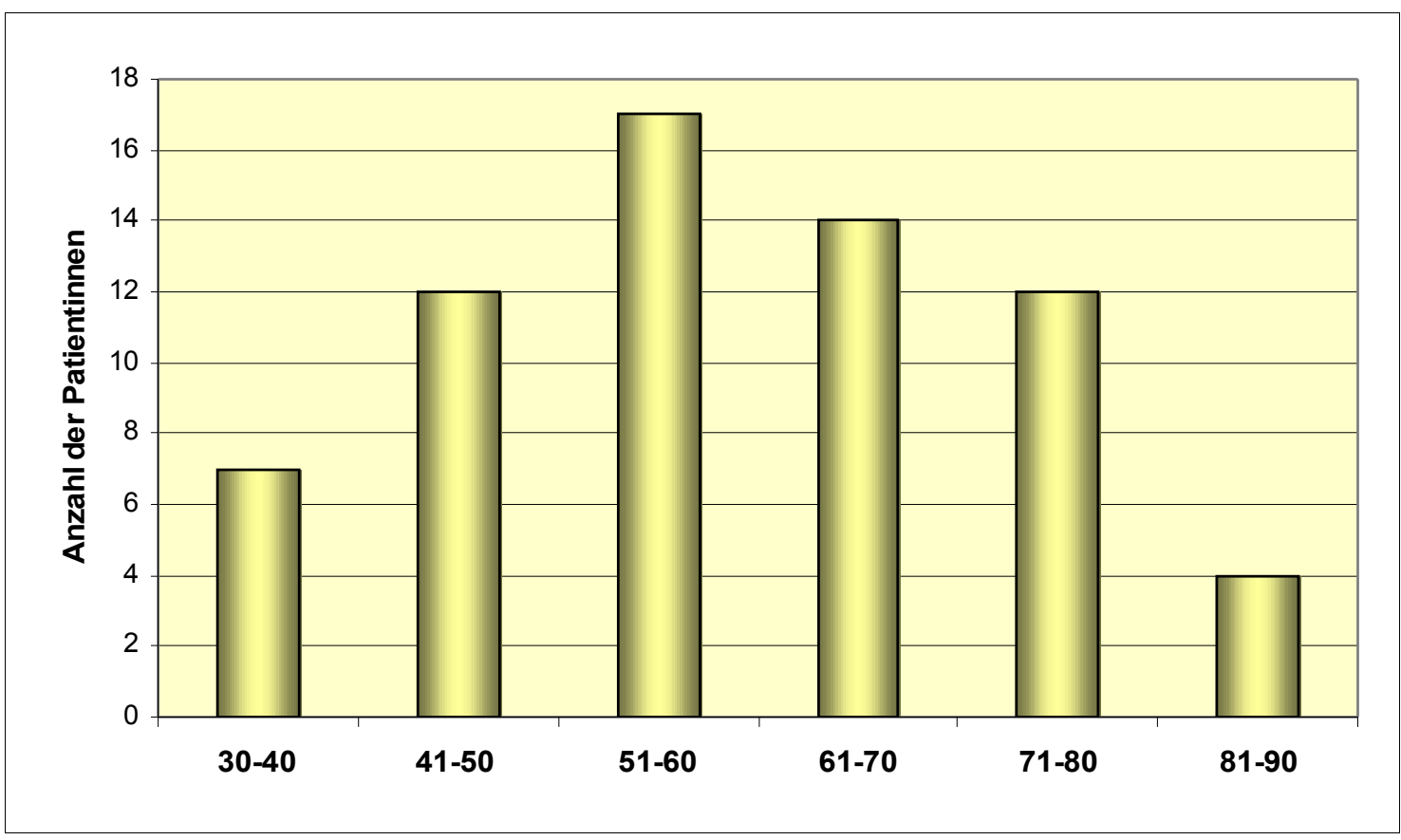

Abbildung 4. Altersverteilung der Patientinnen zum Zeitpunkt der Operation 


\subsection{Dauer des Krankenhausaufenthaltes}

Die Dauer des Krankenhausaufenthaltes betrug durchschnittlich 28,9 Tage, die Aufenthaltsdauer auf der Intensivstation 1,8 Tage.

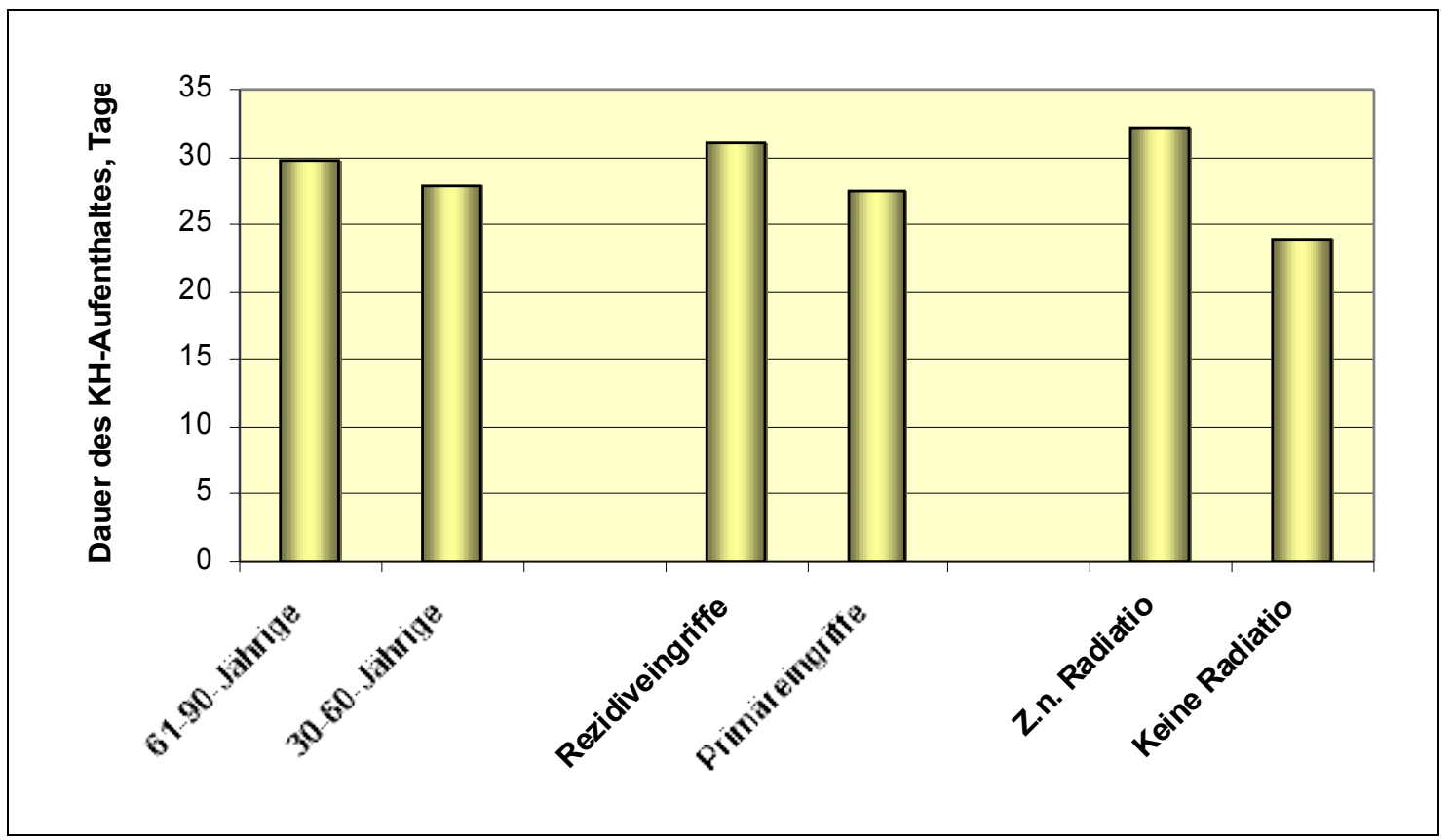

Abbildung 5. Dauer des Krankenhausaufenthaltes in verschiedenen Patientinnengruppen

Die Dauer des Krankenhausaufenthaltes war kürzer in der Gruppe von Patientinnen bis 60 Jahren, nach Primäreingriffen und in der Gruppe der Patientinnen ohne vorangegangene Radiatio, wobei der Unterschied zwischen den Gruppen statistisch nicht signifikant ist. 


\begin{tabular}{|c|c|c|c|}
\hline & \multicolumn{2}{|c|}{ Altersgruppe } & \multirow{2}{*}{ Signifikanz } \\
\cline { 2 - 3 } & $\mathrm{c} \begin{array}{c}61-90 \text { Jahre } \\
\mathrm{n}=30\end{array}$ & $\begin{array}{c}30-60 \text { Jahre } \\
\mathrm{n}=36\end{array}$ & $\mathrm{p}=0,077$ \\
\hline $\begin{array}{c}\text { Dauer des Krankenhaus- } \\
\text { aufenthaltes, Tage }\end{array}$ & $29,8( \pm 1,5)$ & $27,8( \pm 2,6)$ & \\
\hline
\end{tabular}

Tabelle 1. Dauer des Krankenhausaufenthaltes in zwei Altersgruppen

\begin{tabular}{|c|c|c|c|}
\hline & $\begin{array}{c}\text { Rezidiveingriff } \\
\mathrm{n}=39\end{array}$ & $\begin{array}{c}\text { Primäreingriff } \\
\mathrm{n}=27\end{array}$ & Signifikanz \\
\hline $\begin{array}{c}\text { Dauer des Krankenhaus- } \\
\text { aufenthaltes, Tage }\end{array}$ & $31,1( \pm 2,6)$ & $27,4( \pm 1,6)$ & $\mathrm{p}=0,151$ \\
\hline
\end{tabular}

Tabelle 2. Dauer des Krankenhausaufenthaltes bei Primär- und Rezidiveingriffen

\begin{tabular}{|c|c|c|c|}
\hline & $\begin{array}{c}\text { Keine Radiatio } \\
\mathrm{n}=40\end{array}$ & $\begin{array}{c}\text { Präoperative } \\
\text { Radiatio } \\
\mathrm{n}=26\end{array}$ & Signifikanz \\
\hline $\begin{array}{c}\text { Dauer des Krankenhaus- } \\
\text { aufenthaltes, Tage }\end{array}$ & $23,9( \pm 1,8)$ & $32,1( \pm 1,8)$ & $\mathrm{p}=0,264$ \\
\hline
\end{tabular}

Tabelle 3. Dauer des Krankenhausaufenthaltes in der Patientinnengruppe mit präoperativer Radiatio und in der Gruppe ohne vorangegangene Radiatio 


\subsection{Operationsindikation und Operationsverfahren}

Im Zeitraum von 01.01.1991 bis 31.12.2005 wurden insgesamt 66 Patientinnen operiert. Bei 65 Patientinnen handelte es sich um gynäkologische Malignome, eine Patientin wurde wegen eines Leiomyosarkoms des Rektums operiert.

Am häufigsten traten Zervixkarzinome (38 Patientinnen) auf, gefolgt von Corpus-CA (8), sowie Vaginal-CA (6), Ovarial-CA (6) und Vulva-CA (5).

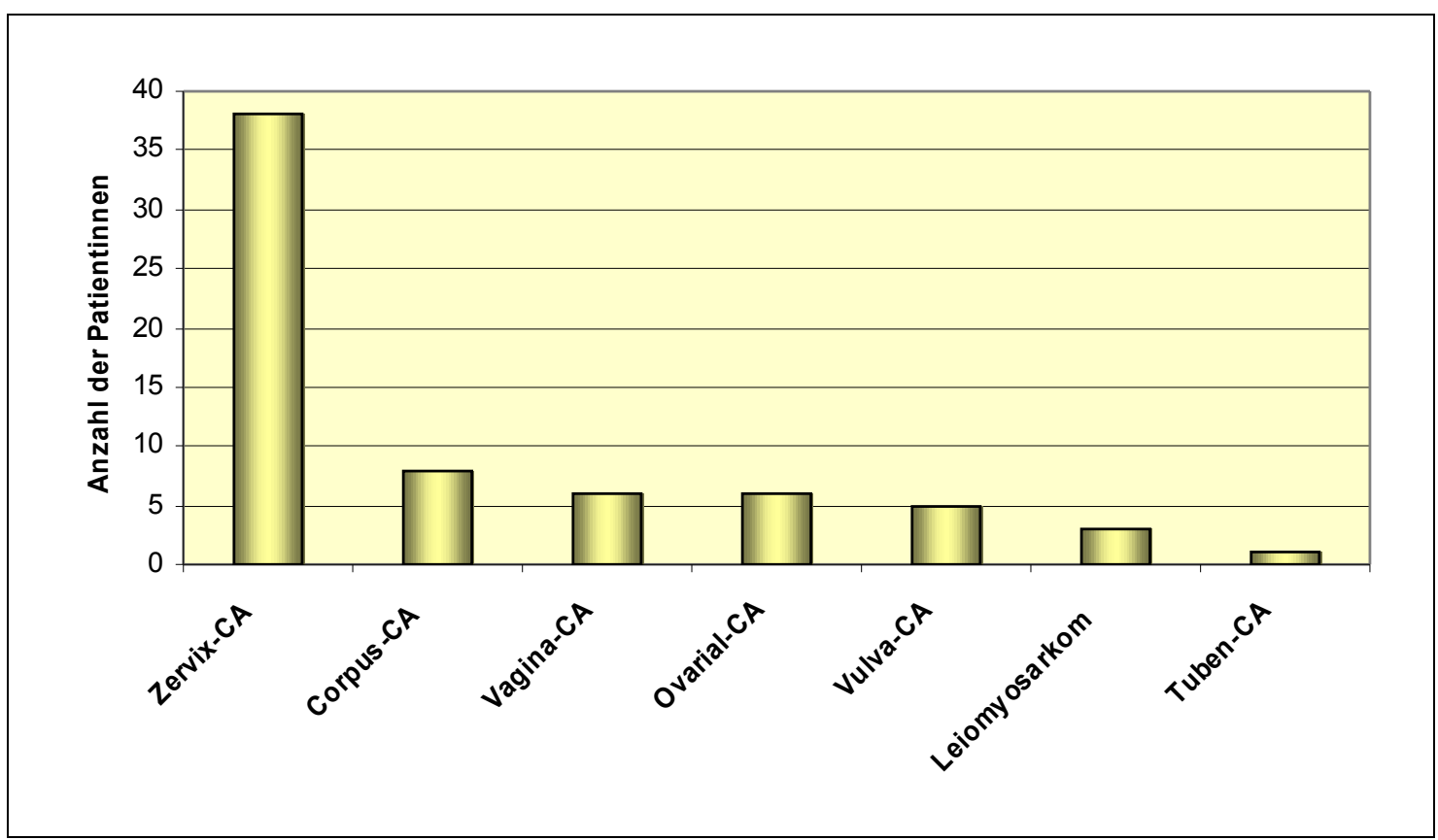

Abbildung 6. Verteilung der Tumorarten im Patientinnenkollektiv 
Je nach Lokalisation wurde mit der entsprechenden Exenterationsart therapiert: 33 vordere Exenterationen, 24 totale Exenterationen und 9 hintere Exenterationen.

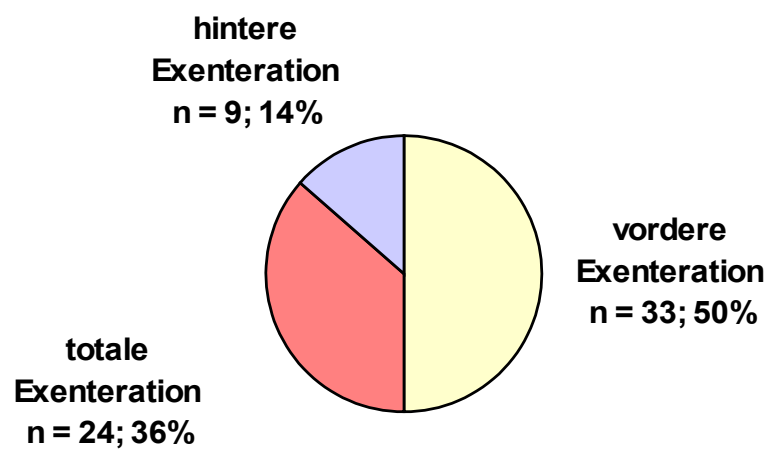

Abbildung 7. Verteilung der Exenterationsarten im Patientinnenkollektiv

Die Indikation zur Exenteration ergab sich in 39 Fällen (59\%) aufgrund eines Rezidivs nach vorangegangener systemischer oder operativer Therapie. In 27 Fällen (41\%) handelte es sich um die Entfernung des Primärtumors.

\begin{tabular}{|c|c|c|}
\hline & Rezidiveingriffe $(\mathrm{n}=39)$ & Primäreingriffe $(\mathrm{n}=27)$ \\
\hline Operation & 39 & $\begin{array}{c}5 \text { (explorativ oder zur } \\
\text { histologischen Sicherung) }\end{array}$ \\
\hline Radiatio & 17 & -- \\
\hline Radiochemotherapie & 2 & 7 \\
\hline Chemotherapie & 6 & 1 \\
\hline
\end{tabular}

Tabelle 4. Vorangegangene Therapie 
In neun von 66 Fällen wurde die Exenteration als palliativer Eingriff vorgenommen. Dabei handelt es sich in fünf Fällen um einen Rezidiveingriff, in zwei Fällen um einen Eingriff nach einer vorangegangenen Radiochemotherapie, in zwei Fällen wurde die palliative Exenteration als ein primärer Eingriff durchgeführt. Am häufigsten wurde der Eingriff bei einer bestehenden Kloake oder bei einer drohenden Kloakenbildung durchgeführt. Weitere Indikationen waren massive rezidivierende Blasenblutungen sowie renale Komplikationen.

\begin{tabular}{|c|c|c|c|c|}
\hline $\begin{array}{l}\text { Pat. } \\
\text { ID }\end{array}$ & Karzinom & $\begin{array}{l}\text { Vorausgegangene } \\
\text { Therapie }\end{array}$ & OP-Indikation & Verlauf \\
\hline $\begin{array}{l}\text { A.M., } \\
43 \mathrm{~J} .\end{array}$ & Zervix-CA & $\begin{array}{l}\text { Neoadjuvante Radio- } \\
\text { Chemotherapie }\end{array}$ & $\begin{array}{l}\text { Rezidivierende PNS-Disloka- } \\
\text { tionen, permanente PNS- } \\
\text { Wechsel, dadurch starke psy- } \\
\text { chische Belastung }\end{array}$ & $\begin{array}{l}\text { Progression der Er- } \\
\text { krankung, } \\
12 \text { Monate später } \\
\text { verstorben }\end{array}$ \\
\hline $\begin{array}{l}\text { B.F., } \\
85 \mathrm{~J} .\end{array}$ & $\begin{array}{l}\text { Rezidiv eines } \\
\text { Corpus-CA }\end{array}$ & $\begin{array}{l}\text { Abdominale HE mit } \\
\text { Adnektomie bds. }\end{array}$ & $\begin{array}{l}\text { Rezidivierende massive Bla- } \\
\text { senblutungen }\end{array}$ & $\begin{array}{l}\text { Postoperative gute } \\
\text { Lebensqualität, } \\
10 \text { Monate später } \\
\text { verstorben }\end{array}$ \\
\hline $\begin{array}{l}\text { H.H., } \\
67 \text { J. }\end{array}$ & $\begin{array}{l}\text { Rezidiv eines } \\
\text { Zervixstumpf-CA }\end{array}$ & $\begin{array}{l}\text { Suprazervikale HE, Ra- } \\
\text { diatio bei } \\
\text { Zervixstumpf-CA }\end{array}$ & Kloakenbildung & $\begin{array}{l}\text { Progression der Er- } \\
\text { krankung, } \\
12 \text { Monate später } \\
\text { verstorben }\end{array}$ \\
\hline $\begin{array}{l}\text { K.Y., } \\
51 \mathrm{~J} .\end{array}$ & $\begin{array}{l}\text { Rezidiv eines } \\
\text { Corpus-CA }\end{array}$ & $\begin{array}{l}\text { Abdominale HE mit } \\
\text { Adnektomie bds., pelvi- } \\
\text { ne und paraaortale LNE }\end{array}$ & $\begin{array}{l}\text { Drohende Kloakenbildung, } \\
\text { massive Blasenblutung }\end{array}$ & $\begin{array}{l}\text { Progression der Er- } \\
\text { krankung, } \\
6 \text { Monate später } \\
\text { verstorben }\end{array}$ \\
\hline $\begin{array}{l}\text { M.H., } \\
68 \mathrm{~J} .\end{array}$ & Zervix-CA & $\begin{array}{l}\text { Kombinierte Radioche- } \\
\text { motherapie }\end{array}$ & $\begin{array}{l}\text { Kloakenbildung, starker Lei- } \\
\text { densdruck }\end{array}$ & $\begin{array}{l}\text { Progression der Er- } \\
\text { krankung, } \\
10 \text { Monate später } \\
\text { verstorben } \\
\end{array}$ \\
\hline $\begin{array}{l}\text { O.C., } \\
49 \mathrm{~J} .\end{array}$ & Leiomyosarkom & keine & $\begin{array}{l}\text { Kloakenbildung, starker Lei- } \\
\text { densdruck }\end{array}$ & $\begin{array}{l}\text { Progression der Er- } \\
\text { krankung, } \\
12 \text { Monate später } \\
\text { verstorben }\end{array}$ \\
\hline $\begin{array}{l}\text { R.M., } \\
50 \text { J. }\end{array}$ & $\begin{array}{l}\text { Zervix-CA mit pe- } \\
\text { ritonealer } \\
\text { Metastasierung }\end{array}$ & keine & $\begin{array}{l}\text { Ausgeprägte Nierenstau mit } \\
\text { Verlust der Nierenfunktion, } \\
\text { starker Leidensdruck }\end{array}$ & Unbekannt \\
\hline $\begin{array}{l}\text { S.L., } \\
70 \mathrm{~J} .\end{array}$ & $\begin{array}{l}\text { Rezidiv eines } \\
\text { Ovarial-CA }\end{array}$ & $\begin{array}{l}\text { Abd. HE mit Adnekto- } \\
\text { mie bds., } \\
\text { Polychemotherapie }\end{array}$ & $\begin{array}{l}\text { Massive Blasenblutungen, Bla- } \\
\text { sentamponaden }\end{array}$ & Unbekannt \\
\hline $\begin{array}{l}\text { W.M., } \\
47 \text { J. }\end{array}$ & $\begin{array}{l}\text { Rezidiv eines } \\
\text { Zervix-CA }\end{array}$ & $\begin{array}{l}\text { Kombinierte RCT, OP } \\
\text { nach Piver III, } \\
\text { postoperative Boost-Be- } \\
\text { strahlung }\end{array}$ & $\begin{array}{l}\text { Kloakenbildung, ausgeprägte } \\
\text { Nierenstau mit Verlust der } \\
\text { Nierenfunktion, starker Lei- } \\
\text { densdruck }\end{array}$ & $\begin{array}{l}\text { Progression der Er- } \\
\text { krankung, } \\
8 \text { Monate später } \\
\text { verstorben }\end{array}$ \\
\hline
\end{tabular}

Tabelle 5. Indikation und Verlauf bei palliativer Exenteration 


\subsection{Histologie}

Bei 37 Patientinnen $(56,1 \%)$ wurde der Tumor in sano reseziert, bei 29 Patientinnen $(42,9 \%)$ erfolgte eine non in sano-Resektion. In der Gruppe der Patientinnen, die präoperativ eine Radiatio erhalten haben, bei älteren Patientinnen sowie bei einer Exenteration mit einem palliativen Einsatz war die Rate der R0-Resektionen geringer.

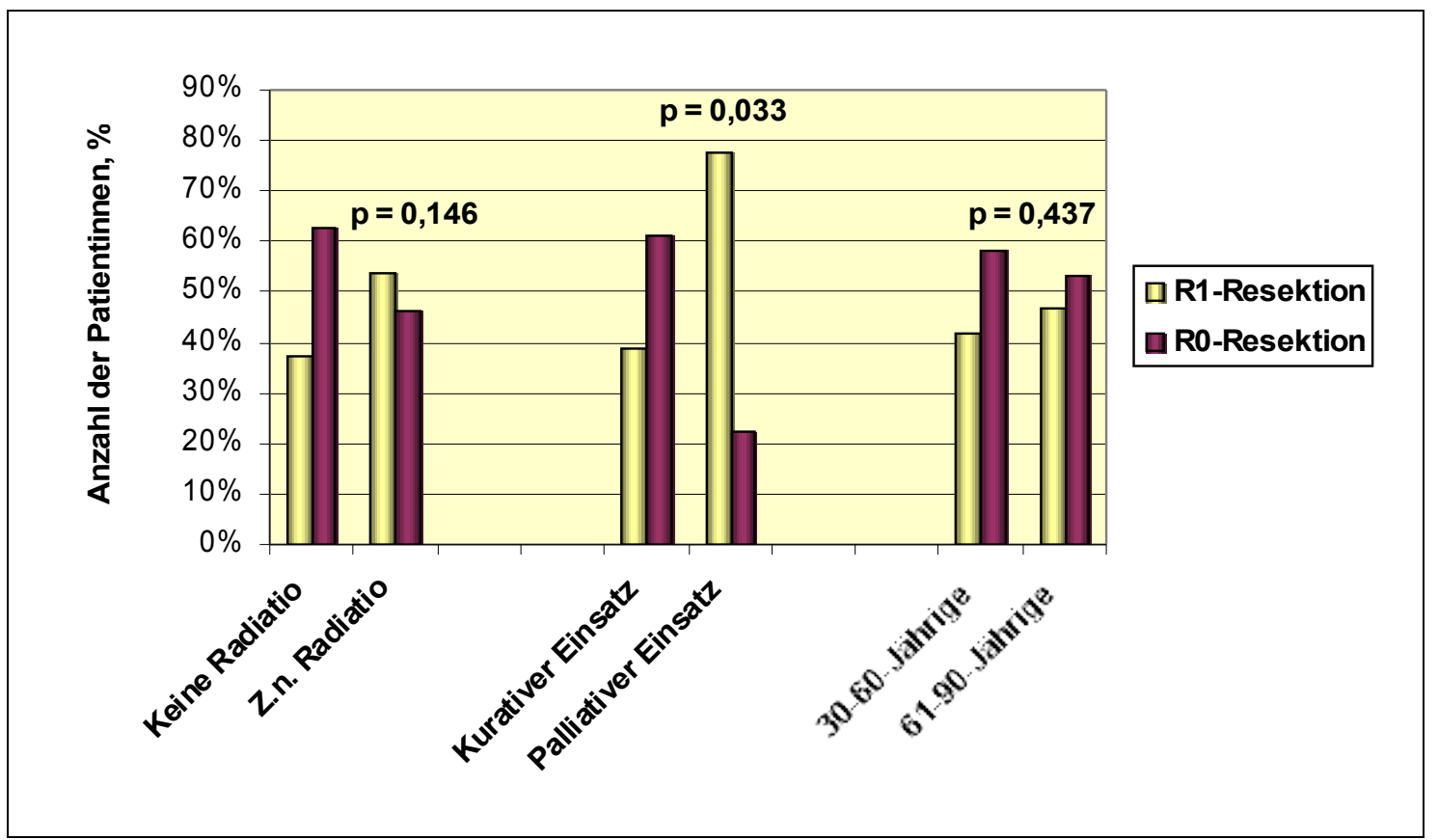

Abbildung 8. R0/R1-Resektionsraten in verschiedenen Patientinnengruppen

In 42 Fällen $(63,6 \%)$ wurde eine Lymphonodektomie im Rahmen der Exenteration durchgeführt, 16 Patientinnen (24,2\%) haben eine Lymphonodektomie schon bei den vorausgegangenen operativen Eingriffen erhalten. Bei 19 exenterierten Patientinnen (45\%) konnte ein Lymphknotenbefall nachgewiesen werden, in elf Fällen mit zusätzlicher Lymphangiosis carcinomatosa. 
Histologisch konnte bei 41 Patientinnen (62\%) eine Blaseninfiltration nachgewiesen werden, davon in 20 Fällen bei vorderer Exenteration und in 19 Fällen bei totaler Exenteration. Ein histologisch nachgewiesener Darmbefall zeigte sich in 20 Fällen (30,3\%), davon bei hinterer Exenteration in drei Fällen und in 17 Fällen bei totaler Exenteration.

\begin{tabular}{|c|c|c|c|}
\hline & $\begin{array}{c}\text { Vordere } \\
\text { Exenteration } \\
(\mathrm{n}=33)\end{array}$ & $\begin{array}{c}\text { Totale } \\
\text { Exenteration } \\
(\mathrm{n}=24)\end{array}$ & $\begin{array}{c}\text { Hintere } \\
\text { Exenteration } \\
(\mathrm{n}=9)\end{array}$ \\
\hline Blaseninfiltration & $22(33,3 \%)$ & $19(79,2 \%)$ & -- \\
\hline Rektuminfiltration & - & $17(70,8 \%)$ & $3(33,3 \%)$ \\
\hline
\end{tabular}

Tabelle 6. Histologisch nachgewiesener Befall der Nachbarorgane bei verschiedenen Exenterationsarten

\subsection{Rekonstruktion}

\subsubsection{Rektumrekonstruktion}

In 33 Fällen wurde ein Eingriff am Colon vorgenommen. Es handelte sich entweder um kontinenzerhaltende Anastomosen $(n=5 ; 15,1 \%)$ oder um einen Anus praeter naturalis $(\mathrm{n}=28 ; 84,9 \%)$. 


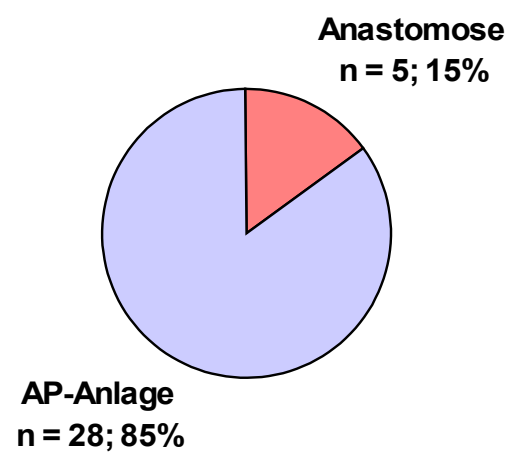

Abbildung 9. Rekonstruktion nach Darmeingriffen

\subsubsection{Harnableitung}

Eingriffe zur Harnableitung waren in 58 Fällen notwendig, dabei haben 44 Patientinnen $(75,9 \%)$ ein Ileum-Conduit erhalten, bei 14 Patientinnen $(24,1 \%)$ erfolgte eine kontinente Harnableitung.

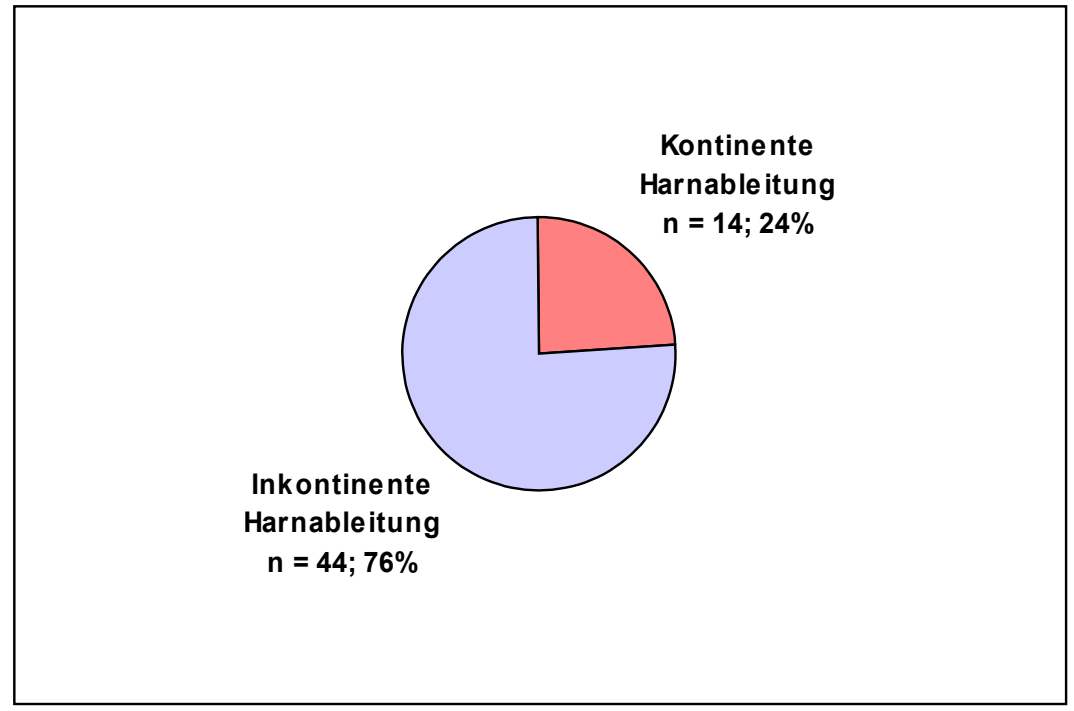

Abbildung 10. Rekonstruktion nach Blaseneingriffen 


\subsection{Operationsverlauf}

Die Operationsdauer betrug im Durchschnitt 339 Minuten $(180-600$ Minuten, s $=90$ $\min )$.

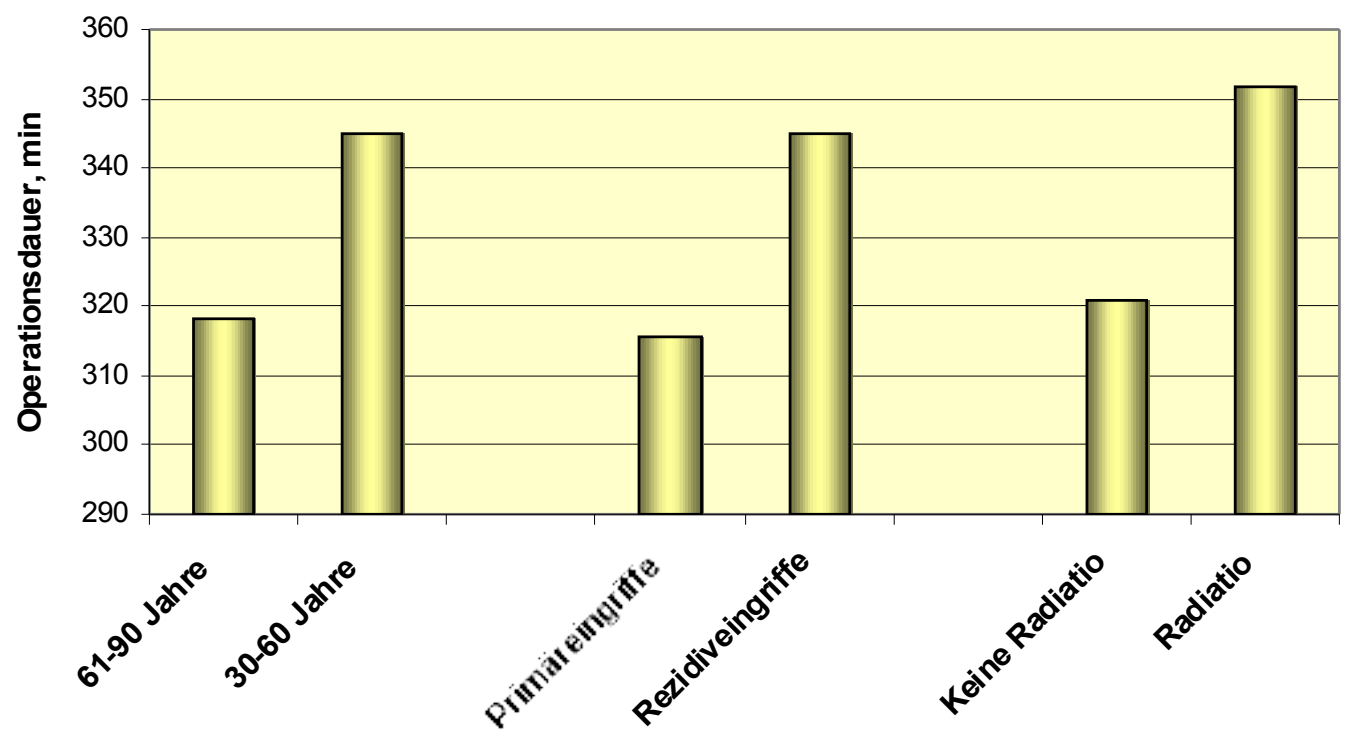

Abbildung 11. Operationsdauer in verschiedenen Patientinnengruppen 
Die Operation hat bei Rezidiveingriffen statistisch signifikant länger gedauert. In der Gruppe von Patientinnen, die präoperativ eine Radiatio erhalten haben und in der Altersgruppe von 30 bis 60 Jahren hat die Operation etwas länger gedauert, der Unterschied war allerdings statistisch nicht signifikant.

\begin{tabular}{|l|c|c|c|}
\hline & \multicolumn{2}{|c|}{ Altersgruppe } & \multirow{2}{*}{ Signifikanz } \\
\cline { 2 - 3 } & $30-60$ Jahre & $61-90$ Jahre \\
$\mathrm{n}=36$ & $\mathrm{n}=30$ & \\
\hline Operationsdauer, $\min$ & $345,1( \pm 86)$ & $318,2( \pm 93)$ & $\mathrm{p}=0,365$ \\
\hline
\end{tabular}

Tabelle 7. Operationsdauer in zwei Altersgruppen

\begin{tabular}{|l|c|c|c|}
\hline & $\begin{array}{c}\text { Primäreingriff } \\
\mathrm{n}=27\end{array}$ & $\begin{array}{c}\text { Rezidiveingriff } \\
\mathrm{n}=39\end{array}$ & Signifikanz \\
\hline Operationsdauer, $\min$ & $315,56( \pm 71)$ & $344,9( \pm 100)$ & $\mathrm{p}=0,028$ \\
\hline
\end{tabular}

Tabelle 8. Operationsdauer bei Primär- und Rezidiveingriffen

\begin{tabular}{|c|c|c|c|}
\hline & $\begin{array}{c}\text { Präoperative } \\
\text { Radiatio }\end{array}$ & $\begin{array}{c}\text { Keine Radiatio } \\
\mathrm{n}=26\end{array}$ & Signifikanz \\
& $351,9( \pm 79)$ & $320,5( \pm 95)$ & $\mathrm{p}=0,786$ \\
\hline Operationsdauer, $\min$ & & \\
\hline
\end{tabular}

Tabelle 9. Operationsdauer in der Patientinnengruppe mit präoperativer Radiatio und in der Gruppe ohne vorangegangene Radiatio 
Im Durchschnitt wurden vier Erythrozytenkonzentrate (0 - 16 EK's) benötigt, bei sieben Patientinnen war keine Bluttransfusion erforderlich.

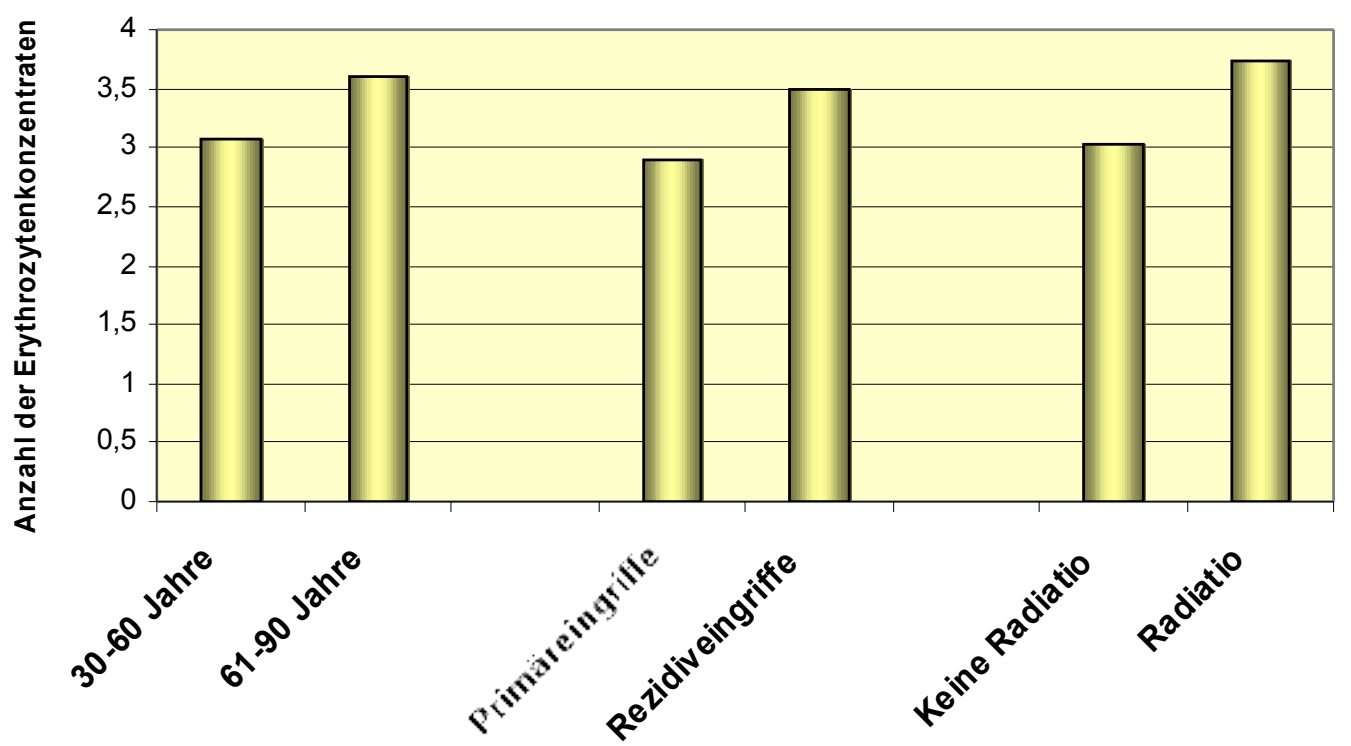

Abbildung 12. Transfusionsmenge in verschiedenen Patientinnengruppen

Die Anzahl der Erythrozytenkonzentrate während der Exenteration lag höher in der Gruppe von Patientinnen, die präoperativ eine Radiatio erhalten haben, bei Rezidiveingriffen und in der Altersgruppe von 61 bis 90 Jahren, wobei sich ein signifikanter statistischer Unterschied zwischen den Gruppen nicht ergab. 


\begin{tabular}{|c|c|c|c|}
\hline & \multicolumn{2}{|c|}{ Altersgruppe } & \multirow{2}{*}{ Signifikanz } \\
\cline { 2 - 3 } & $30-60$ Jahre & $61-90$ Jahre \\
$\mathrm{n}=36$ & $3,6( \pm 2,9)$ & $\mathrm{p}=0,943$ \\
\hline Anzahl der EK's & $3,08( \pm 2,2)$ & 30 & \\
\hline
\end{tabular}

Tabelle 10. Transfusionsmenge in zwei Altersgruppen

\begin{tabular}{|c|c|c|c|}
\hline & $\begin{array}{c}\text { Primäreingriff } \\
\mathrm{n}=27\end{array}$ & $\begin{array}{c}\text { Rezidiveingriff } \\
\mathrm{n}=39\end{array}$ & Signifikanz \\
\hline Anzahl der EK's & $2,9( \pm 3,4)$ & $3,5( \pm 2)$ & $\mathrm{p}=0,245$ \\
\hline
\end{tabular}

Tabelle 11. Transfusionsmenge bei Primär- und Rezidiveingriffen

\begin{tabular}{|c|c|c|c|}
\hline & $\begin{array}{c}\text { Präoperative } \\
\text { Radiatio }\end{array}$ & $\begin{array}{c}\text { Keine Radiatio } \\
\mathrm{n}=26\end{array}$ & Signifikanz \\
& $3,73( \pm 3,4)$ & $3,03( \pm 1,8)$ & $\mathrm{p}=0,077$ \\
\hline Anzahl der EK's & & \\
\hline
\end{tabular}

Tabelle 12. Transfusionsmenge in der Patientinnengruppe mit präoperativer Radiatio und in der Gruppe ohne vorangegangene Radiatio 


\subsection{Morbidität und Mortalität}

Es kam bei insgesamt 40 Patientinnen $(60,6 \%)$ zu postoperativen Komplikationen.

Am häufigsten traten folgende Komplikationen auf:

\begin{tabular}{|l|l|}
\hline & $\begin{array}{l}\text { Anzahl der } \\
\text { Patientinnen }\end{array}$ \\
\hline Chirurgische Komplikationen & \\
\hline Wundheilungssörung & 10 \\
\hline Relaparotomie bei Komplikationen & 7 \\
\hline Anastomoseinsuffizienz & 6 \\
\hline Fistelbildung & 6 \\
\hline Ileus und Subileus & 6 \\
\hline Peritonitis & 3 \\
\hline Revisionsbedürftige Blutung & 1 \\
\hline Lymphocele & 1 \\
\hline Urosepsis & 1 \\
\hline Nierenstau & 1 \\
\hline Nicht-chirurgische Komplikationen & \\
\hline Adynamie und schlechte Erholung & 7 \\
\hline Reaktive Depression & 3 \\
\hline Neurologische Ausfälle & 2 \\
\hline Blasenentleerungsstörung & 2 \\
\hline Respiratorische Insuffizienz & 2 \\
\hline Thrombose & 2 \\
\hline Lymphödem & 2 \\
\hline Harnwegsinfekt & 2 \\
\hline Akute Pankreatitis & 1 \\
\hline Atelektase & 1 \\
\hline & \\
\hline
\end{tabular}

Tabelle 13. Komplikationen im Patientenkollektiv 
Hinsichtlich des Schweregrades der Komplikationen ergab sich die folgende Verteilung:

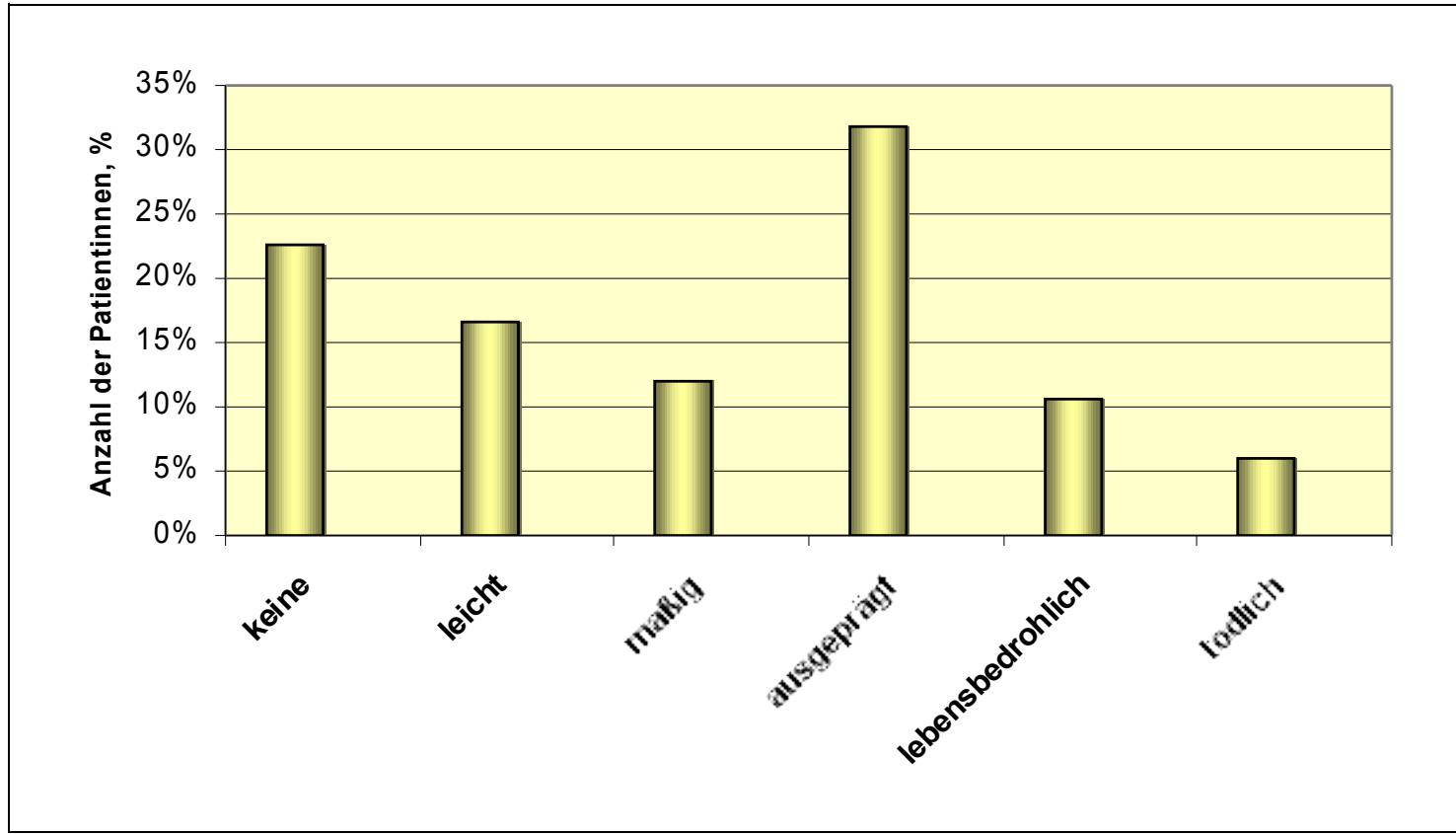

Abbildung 13. Schweregrad der Komplikationen im Patientinnenkollektiv

Betrachtet man den Verlauf bei verschiedenen Arten der pelvinen Exenteration, so treten bei hinterer Exenteration etwas häufiger lebensbedrohliche und tödliche Komplikationen auf, es zeigte sich aber kein signifikanter Unterschied $(\mathrm{p}=0,685)$

\begin{tabular}{|c|c|c|c|}
\hline Komplikationen & $\begin{array}{c}\text { Vordere } \\
\text { Exenteration } \\
\mathrm{n}=33\end{array}$ & $\begin{array}{c}\text { Hintere } \\
\text { Exenteration } \\
\mathrm{n}=9\end{array}$ & $\begin{array}{c}\text { Totale } \\
\text { Exenteration } \\
\mathrm{n}=24\end{array}$ \\
\hline keine & $9(27,3 \%)$ & $1(11,1 \%)$ & $5(20,8 \%)$ \\
\hline leicht & $7(21,2 \%)$ & 0 & $4(16,7 \%)$ \\
\hline mäßig & $2(6,1 \%)$ & $2(22,2 \%)$ & $7(16,7 \%)$ \\
\hline ausgeprägt & $11(33,3 \%)$ & $3(33,3 \%)$ & $4(29,2 \%)$ \\
\hline lebensbedrohlich & $2(6 \%)$ & $2(22,2 \%)$ & $3(12,5 \%)$ \\
\hline tödlich & $2(6 \%)$ & $1(11,1 \%)$ & $1(4,2 \%)$ \\
\hline
\end{tabular}

Tabelle 14. Komplikationen bei verschiedenen Exenterationsarten 


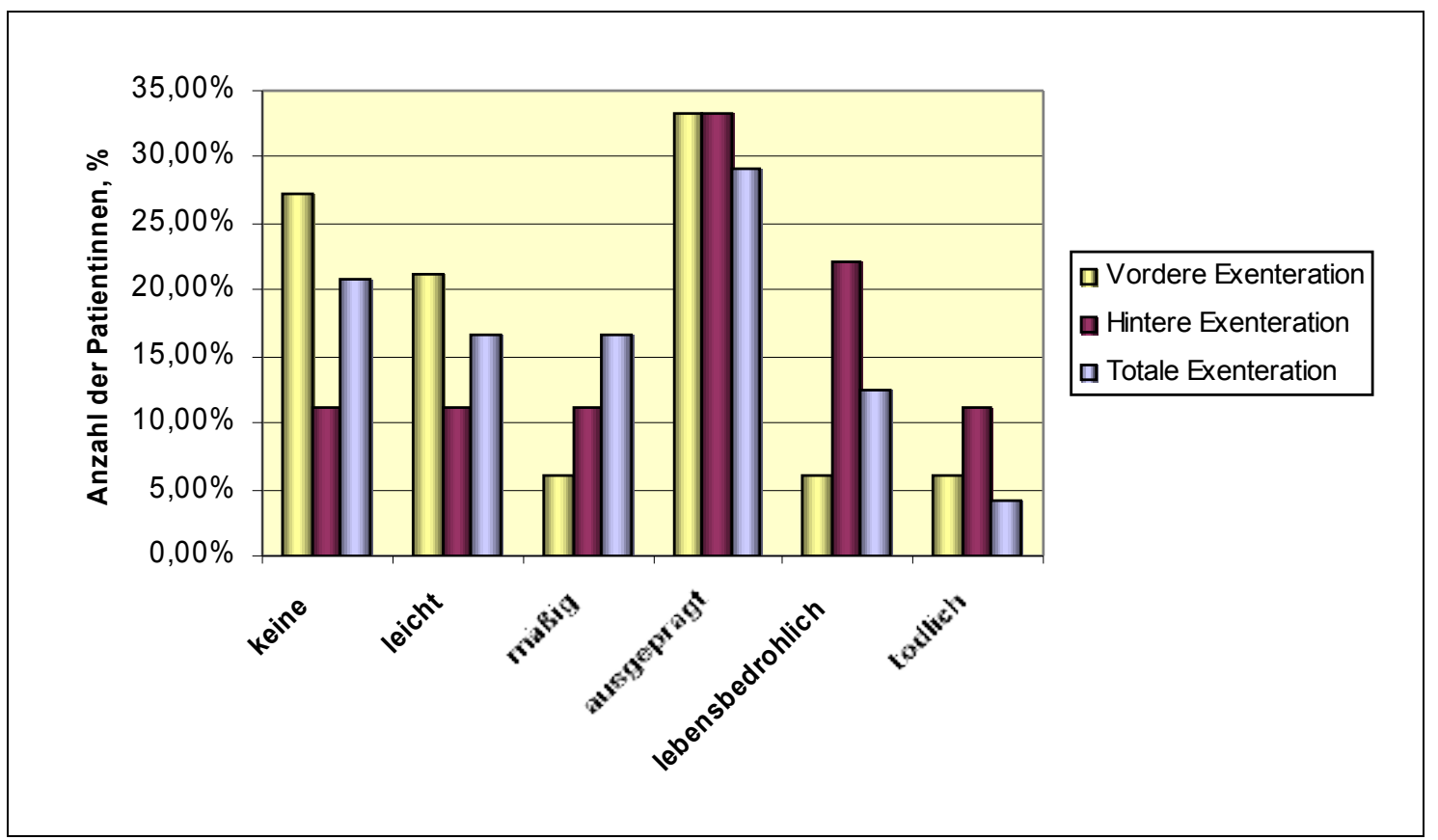

Abbildung 14. Komplikationen bei verschiedenen Exenterationsarten

In der Altersgruppe der 30- bis 60-Jährigen gestaltete sich der postoperative Verlauf häufiger komplikationslos, lebensbedrohliche Komplikationen traten seltener auf. Tödliche Komplikationen traten nur in der Gruppe der 61- bis 90-Jährigen auf. Ein signifikanter Unterschied ergab sich nicht $(\mathrm{p}=0,096)$.

\begin{tabular}{|c|c|c|}
\hline \multirow{2}{*}{ Komplikationen } & \multicolumn{2}{|c|}{ Altersgruppe } \\
\cline { 2 - 3 } & $30-60$ Jahre & $61-90$ Jahre \\
$\mathrm{n}=36$ & $\mathrm{n}=30$ \\
\hline keine & $10(27,8 \%)$ & $5(16,7 \%)$ \\
\hline leicht & $7(19,4 \%)$ & $4(13,3 \%)$ \\
\hline mäßig & $2(5,6 \%)$ & $6(20 \%)$ \\
\hline ausgeprägt & $13(36,1 \%)$ & $8(26,7 \%)$ \\
\hline lebensbedrohlich & $4(11,1 \%)$ & $3(10 \%)$ \\
\hline tödlich & 0 & $4(13,3 \%)$ \\
\hline
\end{tabular}

Tabelle 15. Komplikationen in 2 Altersgruppen 


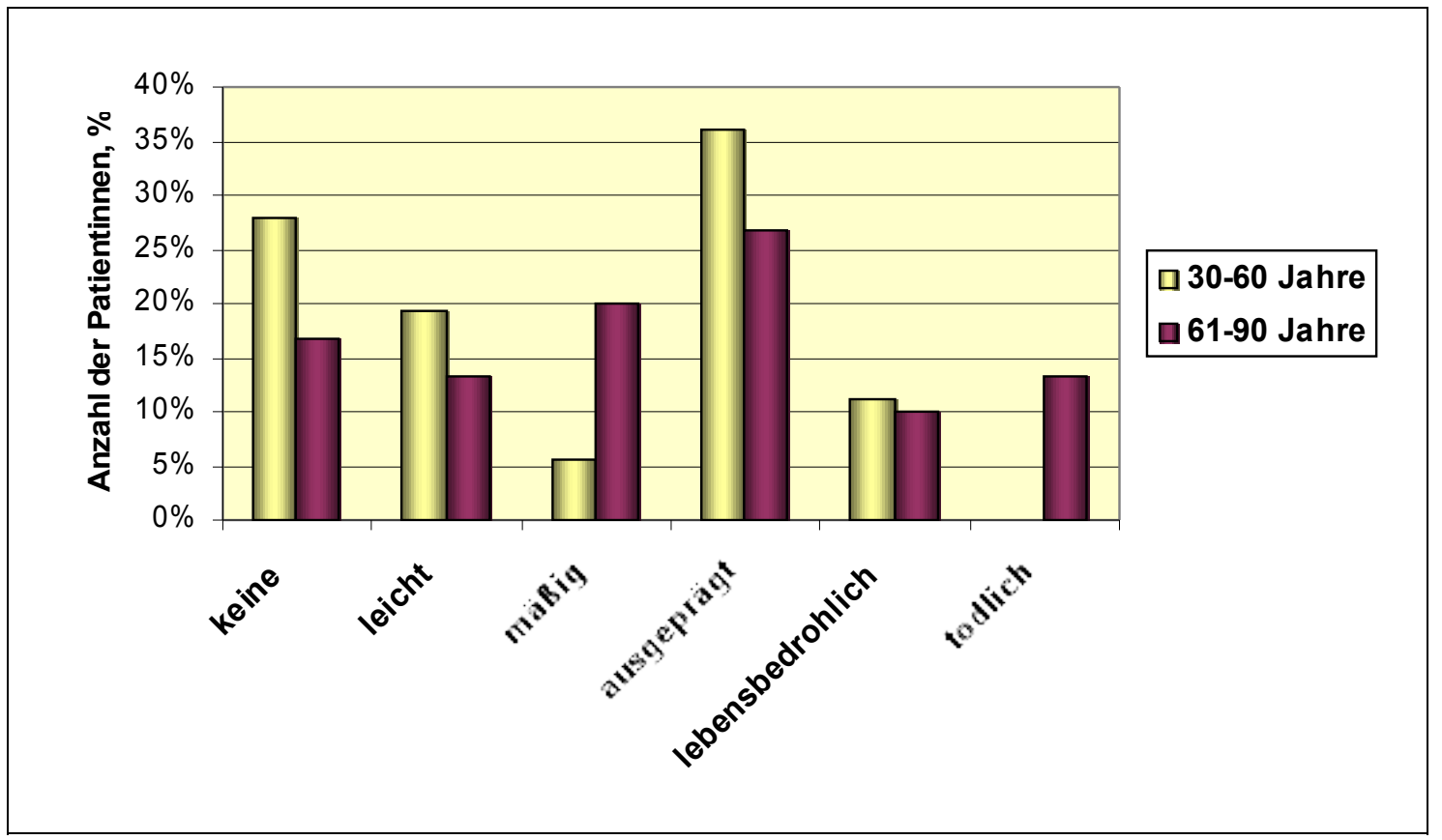

Abbildung 15. Komplikationen in 2 Altersgruppen

Bei den Primäreingriffen gestaltete sich der postoperative Verlauf etwas häufiger komplikationslos, lebensbedrohliche Komplikationen traten seltener auf als bei Rezidiveingriffen. Die tödlichen Komplikationen traten nur bei Rezidiveingriffen auf. Der Unterschied war statistisch nicht signifikant $(\mathrm{p}=0,154)$.

\begin{tabular}{|c|c|c|}
\hline Komplikationen & $\begin{array}{c}\text { Primäreingriff } \\
\mathrm{n}=27\end{array}$ & $\begin{array}{c}\text { Rezidiveingriff } \\
\mathrm{n}=39\end{array}$ \\
\hline keine & $8(29,6 \%)$ & $7(17,9 \%)$ \\
\hline leicht & $3(11,1 \%)$ & $8(20,5 \%)$ \\
\hline mäßig & $4(14,8 \%)$ & $4(10,3 \%)$ \\
\hline ausgeprägt & $11(40,7 \%)$ & $10(25,6 \%)$ \\
\hline lebensbedrohlich & $1(3,7 \%)$ & $6(15,4 \%)$ \\
\hline tödlich & 0 & $4(10,2 \%)$ \\
\hline
\end{tabular}

Tabelle 16. Komplikationen bei Primär- und Rezidiveingriffen 


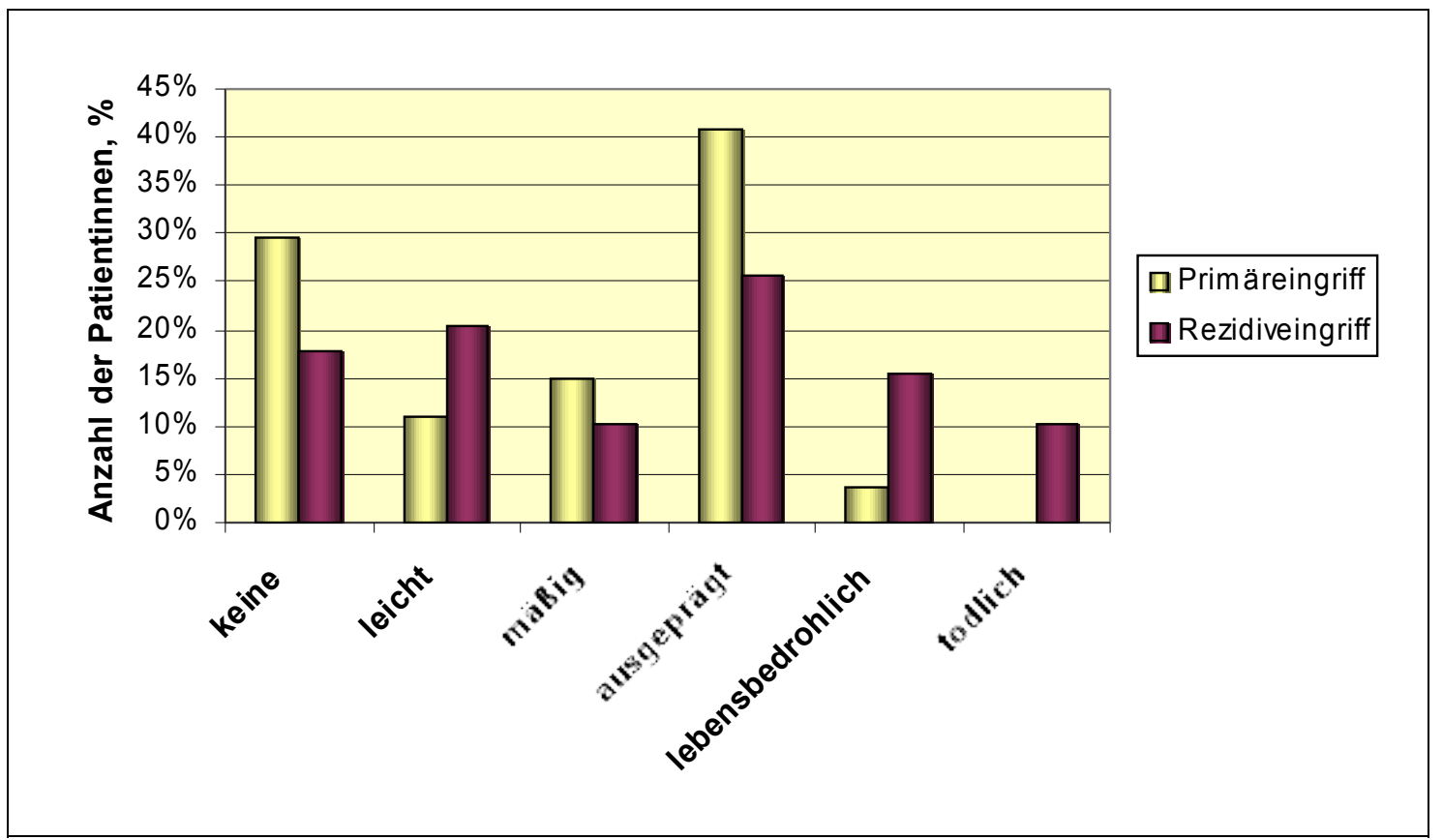

Abbildung 16. Komplikationen bei Primär- und Rezidiveingriffen

In der Patientinnengruppe ohne vorangegangene Radiatio gestaltete sich der postoperative Verlauf etwas häufiger komplikationslos, tödliche Komplikationen traten nur in der Gruppe der Patientinnen mit vorangegangener Radiatio auf. Ein signifikanter Unterschied ergab sich nicht $(p=0,204)$.

\begin{tabular}{|c|c|c|}
\hline Komplikationen & $\begin{array}{c}\text { Präoperative } \\
\text { Radiatio } \\
\mathrm{n}=26\end{array}$ & $\begin{array}{c}\text { Keine Radiatio } \\
\mathrm{n}=40\end{array}$ \\
\hline keine & $5(19,2 \%)$ & $10(25 \%)$ \\
\hline leicht & $5(19,2 \%)$ & $6(15 \%)$ \\
\hline mäßig & $3(11,5 \%)$ & $5(12,5 \%)$ \\
\hline ausgeprägt & $7(26,9 \%)$ & $14(35 \%)$ \\
\hline lebensbedrohlich & $2(7,7 \%)$ & $5(12,5 \%)$ \\
\hline tödlich & $4(15,4 \%)$ & 0 \\
\hline
\end{tabular}

Tabelle 17. Komplikationen in der Patientinnengruppe mit und ohne vorangegangene Radiatio 


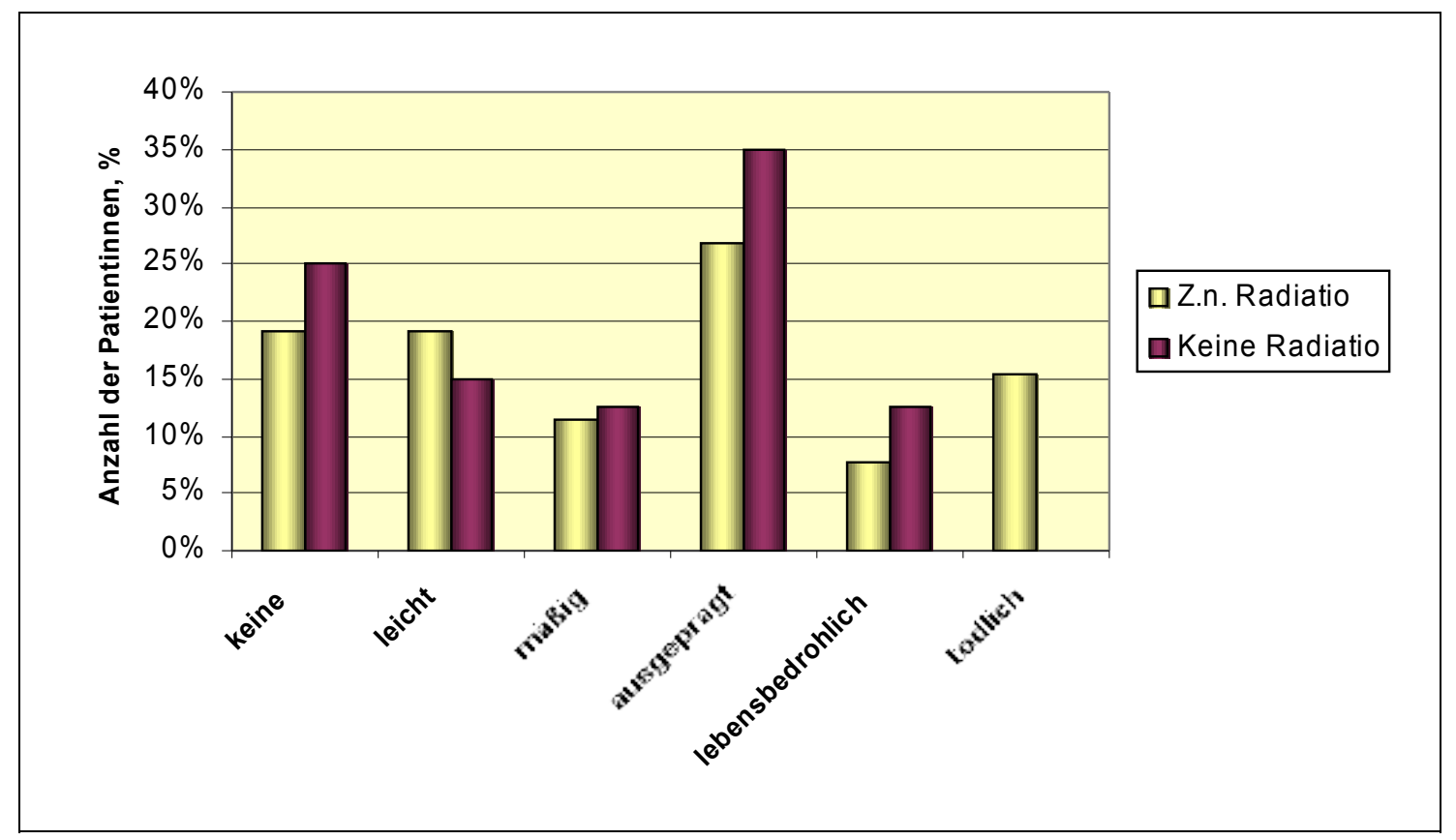

Abbildung 17. Komplikationen in der Patientinnengruppe mit und ohne vorangegangene

\section{Radiatio}

In der Patientinnengruppe, die eine kontinente Harnableitung erhalten haben, traten ausgeprägte und lebensbedrohliche Komplikationen häufiger auf. Tödliche Komplikationen traten nur in der Gruppe der Patientinnen auf, die eine inkontinente Harnableitung erhalten haben. Ein signifikanter Unterschied zwischen den beiden Gruppen ergab sich nicht $(\mathrm{p}=0,706)$.

\begin{tabular}{|c|c|c|}
\hline Komplikationen & $\begin{array}{c}\text { Inkontinente } \\
\text { Harnableitung } \\
\mathrm{n}=44\end{array}$ & $\begin{array}{c}\text { Kontinente } \\
\text { Harnableitung } \\
\mathrm{n}=14\end{array}$ \\
\hline keine & $11(25 \%)$ & $3(21,4 \%)$ \\
\hline leicht & $9(20,5 \%)$ & $2(14,3 \%)$ \\
\hline mäßig & $5(11,4 \%)$ & $1(7,1 \%)$ \\
\hline ausgeprägt & $12(27,3 \%)$ & $6(42,9 \%)$ \\
\hline lebensbedrohlich & $4(9,1 \%)$ & $2(14,3 \%)$ \\
\hline tödlich & $3(6,8 \%)$ & 0 \\
\hline
\end{tabular}

Tabelle 18. Komplikationen bei verschiedenen Harnableitungen 


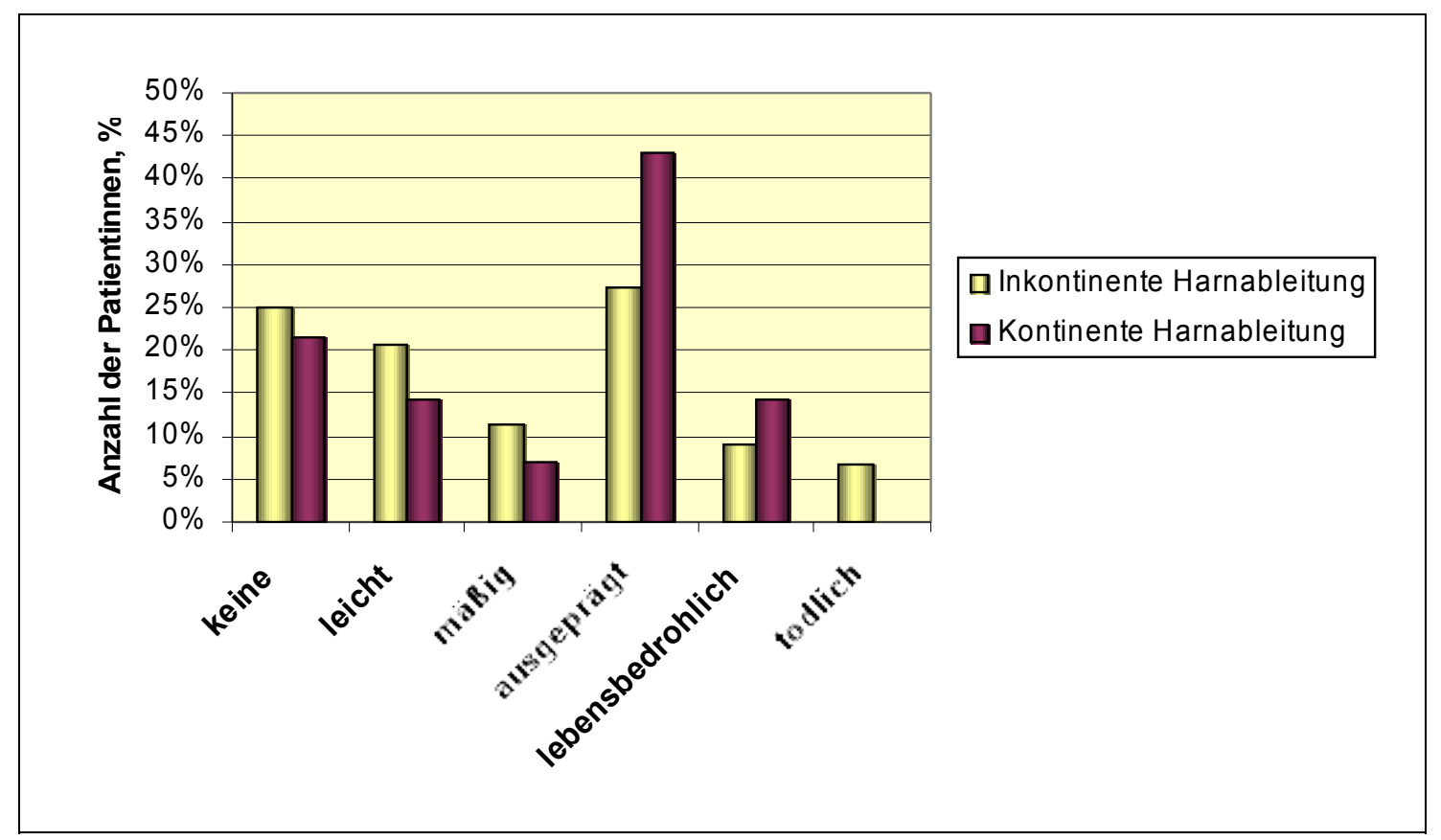

Abbildung 18. Komplikationen bei verschiedenen Harnableitungen

Es gab perioperativ vier Todesfälle, somit beträgt die Mortalität $6 \%$.

Bei einer 74-jährigen Patientin, die wegen eines zweiten Zervixkarzinomrezidivs mit Rektuminfiltration (in der Vorgeschichte eine Wertheim-OP, Radiatio bei einem vaginalen Rezidiv) exenteriert wurde, kam es intraoperativ zu einer unstillbaren Blutung aus der Kreuzbeinhöhle, die schließlich zum Herz-Kreislauf-Versagen und Exitus intabula führte.

Bei der zweiten Patientin wurde eine totale Exenteration bei zweitem Scheidenstumpfrezidiv des Korpuskarzinoms mit Infiltration von Blase und Rektum vorgenommen (in der Vorgeschichte lagen eine abdominale Hysterektomie mit Adnektomie bds. sowie Radiatio bei einem Scheidenstumpfrezidiv vor). Am 6. postoperativen Tag entwickelte die Patientin eine Peritonitis, eine Bauchwandnekrose sowie eine Nekrose des Anus praeters. Daraufhin erfolgten mehrere Revisionslaparotomien mit Lavage. Der Zustand der Patientin konnte trotz der operativen und intensiv-medizinischen 
Maßnahmen nicht positiv beeinflusst werden. Die Sepsis nahm einen fulminanten Verlauf, so dass die Patientin am 34. postoperativen Tag an septisch bedingtem Multiorganversagen verstarb.

Bei der dritten Patientin wurde eine vordere Exenteration bei einem Vaginalkarzinomrezidiv mit Beteiligung der Harnblase durchgeführt (in der Vorgeschichte Kolpektomie mit anschließender Bestrahlung, multiple internistische Vorerkrankungen). Am ersten postoperativen Tag kam es zu einer Thrombose der Arteria iliaca communis links, woraufhin eine sofortige Lyse und eine Stenteinlage, sowie am dritten postoperativen Tag eine Fasziotomie bei einem Kompartmentsyndrom des linken Beines erfolgten. Im weiteren Verlauf kam es trotz aller Maßnahmen zum progredienten Multiorganversagen, an dessen Folgen die Patientin am 7. postoperativen Tag verstarb.

Bei der vierten Betroffenen handelt es sich um eine 64-jährige Patientin, die wegen eines zentralen Rezidivs eines Korpuskarzinoms eine vordere Exenteration erhalten hat (in der Anamnese abdominale Hysterektomie mit Adnektomie mit anschließender kombinierter Radiatio). Aufgrund einer Anastomoseinsuffizienz am Rektum mit lokaler Peritonitis wurde die Patientin am fünften postoperativen Tag relaporotomiert und eine Anlage eines doppelläufigen Transversostomas durchgeführt. Am siebten postoperativen Tag wurde die Patientin ohne suffizienten Kreislauf auf Station aufgefunden. Es erfolgte eine umgehende Reanimationsbehandlung. Im weiteren Verlauf entwickelte die Patientin ein zunehmendes Multiorganversagen und verstarb noch am selben Tag unter dem Bild eines septischen Kreislaufschocks. 


\subsection{Adjuvante Behandlung}

Eine adjuvante Behandlung nach der Exenteration erfolgte in 37 Fällen, in 14 Fällen eine Bestrahlung, in 11 Fällen eine Chemotherapie und in 12 Fällen eine kombinierte Radiochemotherapie.

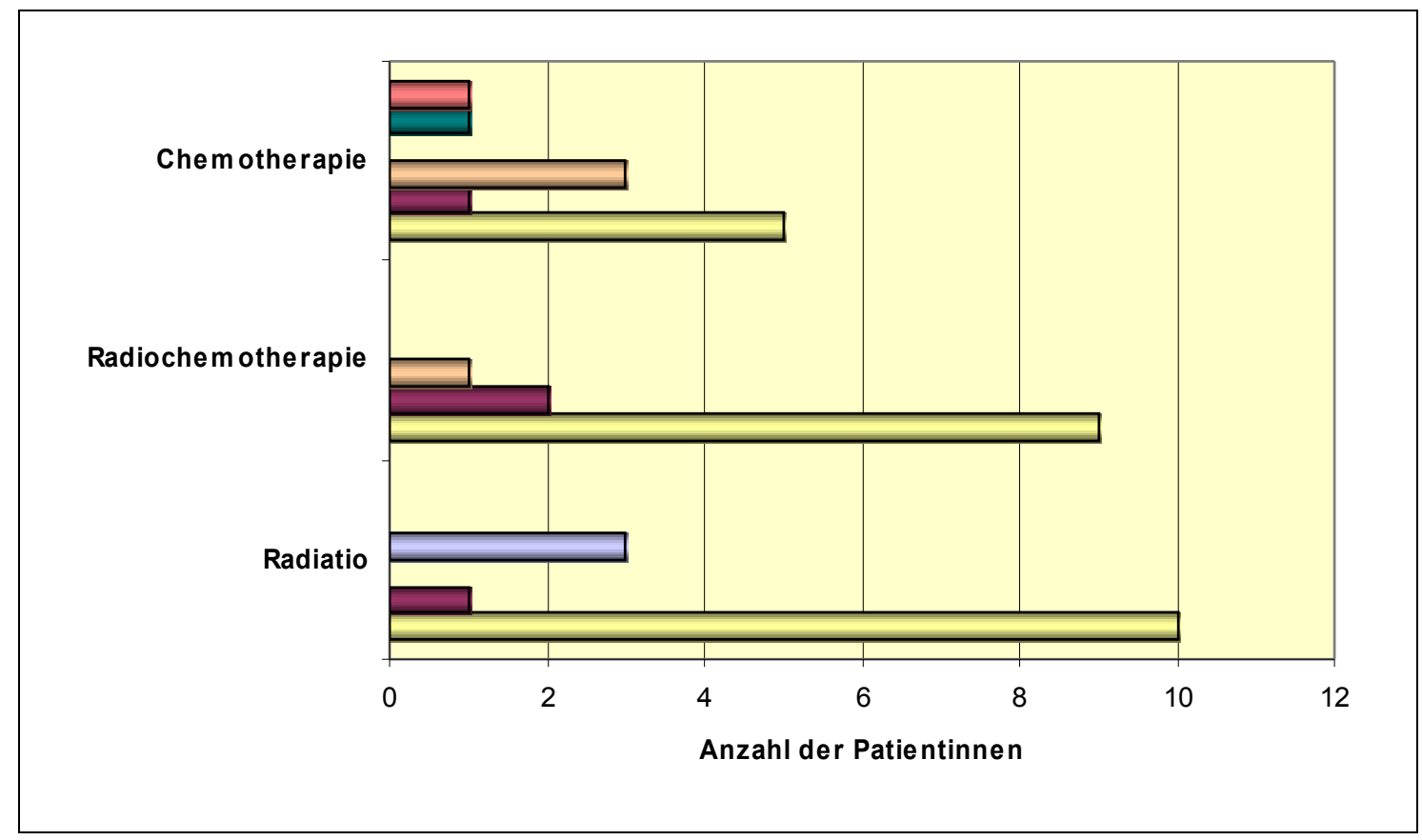

Abbildung 19. Adjuvante Therapie nach Exenteration bei verschiedenen Tumorarten

\subsection{Onkologischer Verlauf}

Während des Beobachtungszeitraumes verstarben 48 Patientinnen, 14 Patientinnen überlebten diesen. In Bezug auf vier Patientinnen sind keine Informationen vorhanden.

Die mittlere Gesamtüberlebenszeit (ab dem Zeitpunkt der Operation) betrug 22 Monate. Die 5-Jahres-Überlebensrate betrug 34\%. 


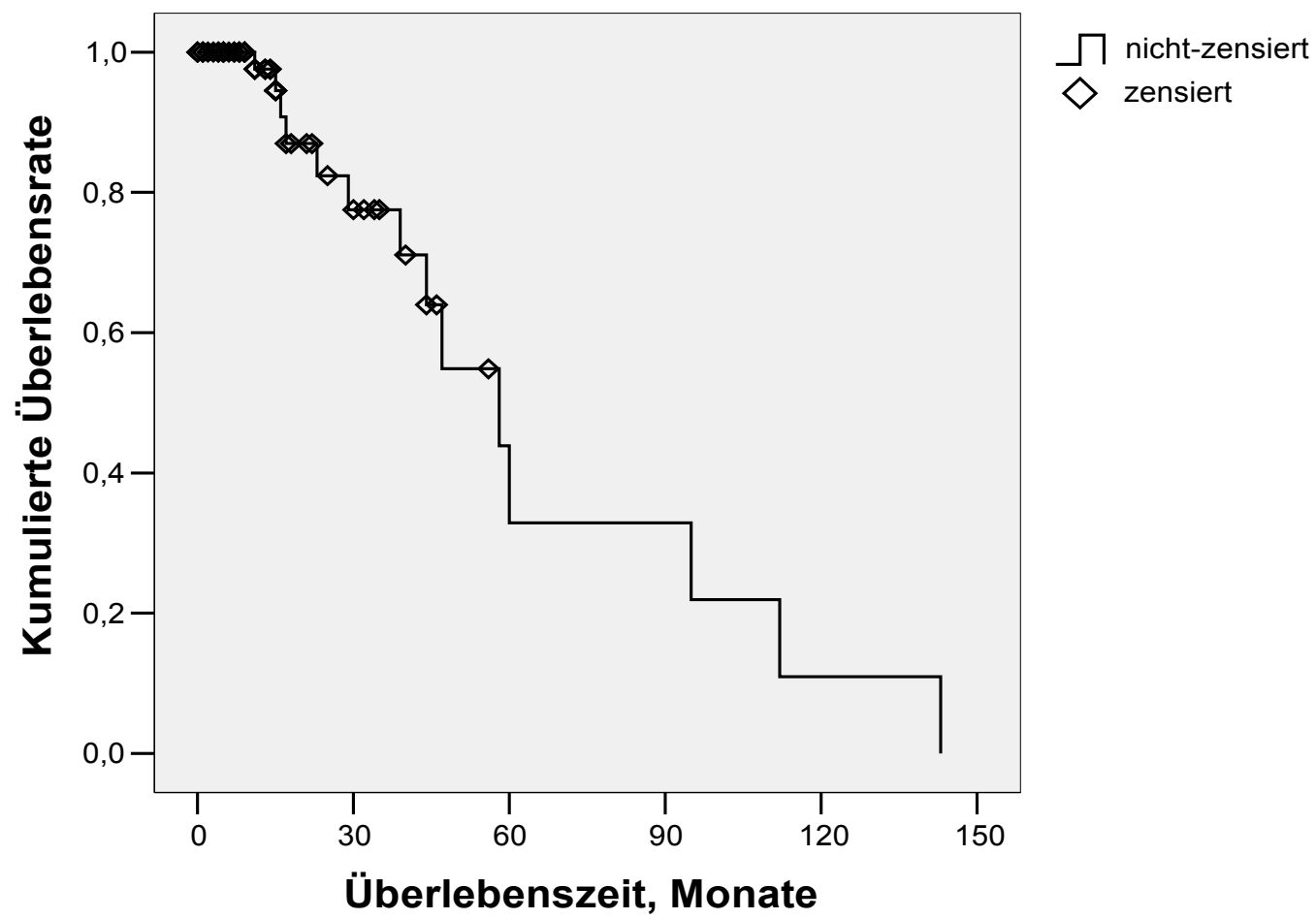

Abbildung 20. Gesamtüberlebenszeit

Bei 37 Patientinnen kam es im Laufe der Beobachtung zur Progression der Erkrankung. Diese äußerte sich in 25 Fällen durch die Entstehung eines Lokalrezidivs, 12 Patientinnen entwickelten Fernmetastasen. Somit liegt die Rezidivrate bei 56\%.

Bei zehn Patientinnen war ein erneuter operativer Eingriff notwendig, vier Patientinnen erhielten eine Chemotherapie und zwei Patientinnen eine Radiatio.

Während des Beobachtungszeitraumes blieben 17 Patientinnen rezidivfrei. Neun von ihnen überlebten diesen Zeitraum rezidivfrei. 
Die mittlere progressionsfreie Überlebenszeit (ab der Operation bis zur Tumorprogression (Lokalrezidiv bzw. Fernmetastasierung)) betrug 17 Monate, die progressionsfreie 5-Jahresüberlebensrate lag bei 19\%.

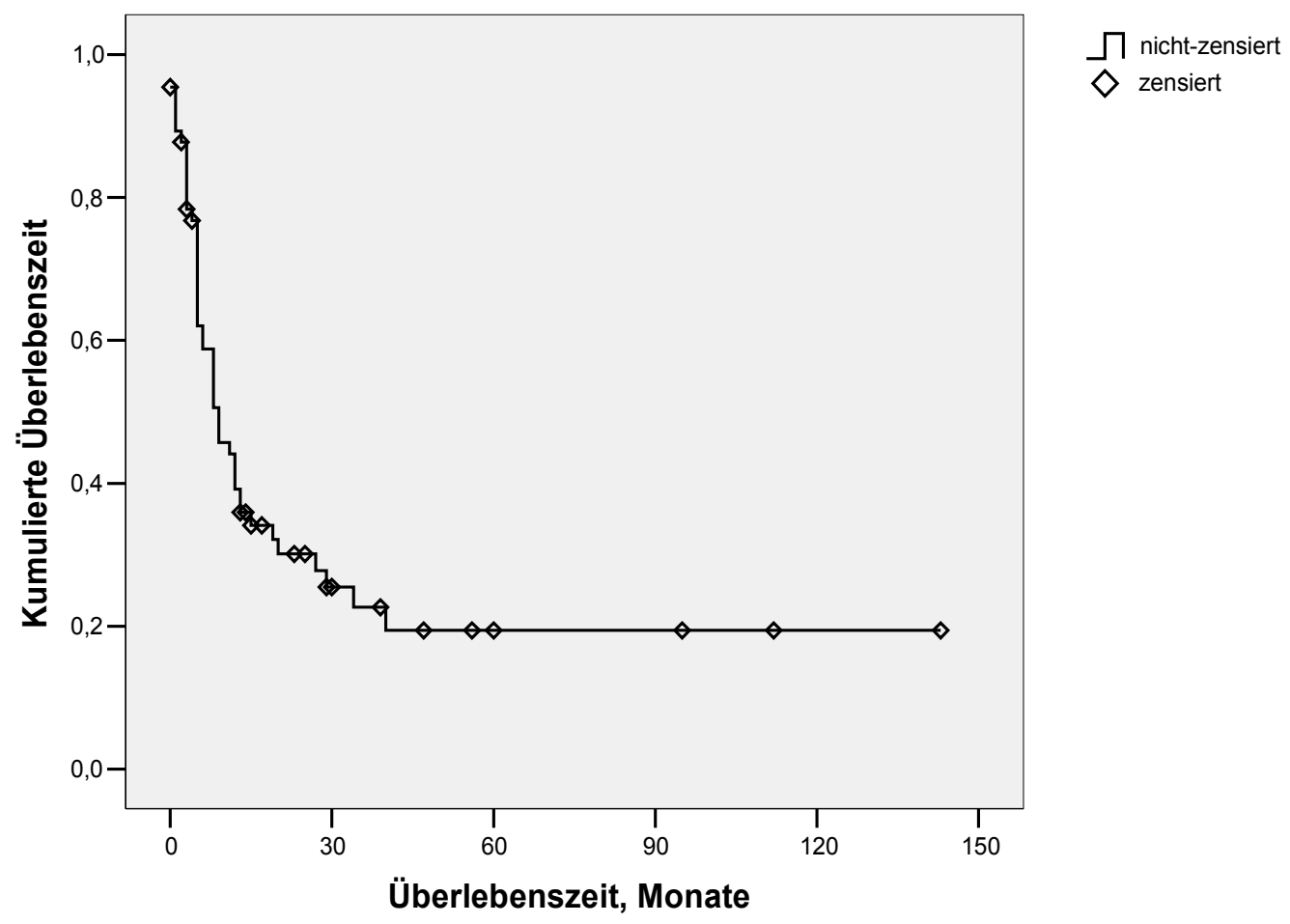

Abbildung 21. Progressionsfreie Überlebenszeit 


\subsection{Einfluss verschiedener Parameter auf die Gesamtüberlebenszeit}

\subsubsection{Einfluss des Nodalstatus}

Die mittlere Gesamtüberlebenszeit bei einem negativen Nodalstatus ( $\mathrm{pN}$ ) betrug 34 Monate. In der Gruppe der Patientinnen mit befallenen Lymphknoten (pN1) betrug diese 16 Monate und in der Gruppe der Patientinnen, bei denen keine Lymphonodektomie im Rahmen der Exenteration durchgeführt wurde ( $\mathrm{pNx}$ ) (entweder auf Grund der bereits durchgeführten Lymphonodektomie bei einem primären Eingriff oder im Rahmen einer palliativen Exenteration) betrug die mittlere Gesamtüberlebenszeit 19 Monate. Die 5-Jahresüberlebensrate lag bei einem negativen Nodalstatus bei $51 \% \mathrm{im}$ Vergleich zu 8\% bei einem N1-Status (in Log-rank Test $p=0,008$ ).

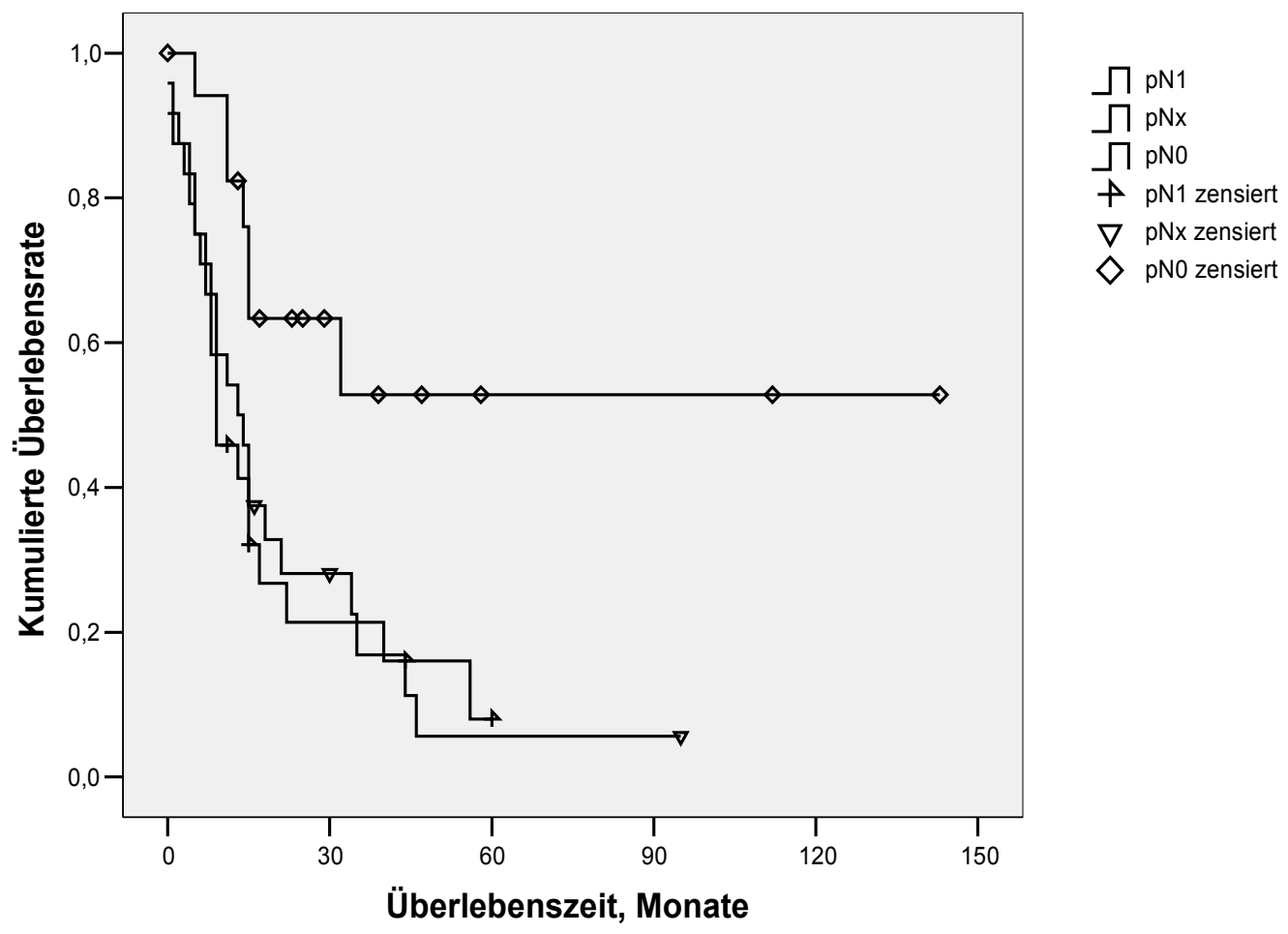

Abbildung 22. Überlebensrate in Abhängigkeit vom Nodalstatus 


\subsubsection{Einfluss der Resektionsart}

Die mittlere Gesamtüberlebenszeit in der Gruppe der Patientinnen, bei denen eine Resektion im Gesunden möglich war, betrug 31 Monate. Bei einer R1-Resektion betrug die mittlere Gesamtüberlebenszeit 11 Monate. Die 5-Jahresüberlebensrate lag bei einer R0-Resektion bei 22\% im Vergleich zu 7\% bei einer R1-Resektion (in Log-rank Test $\mathrm{p}=$ $0,001)$.

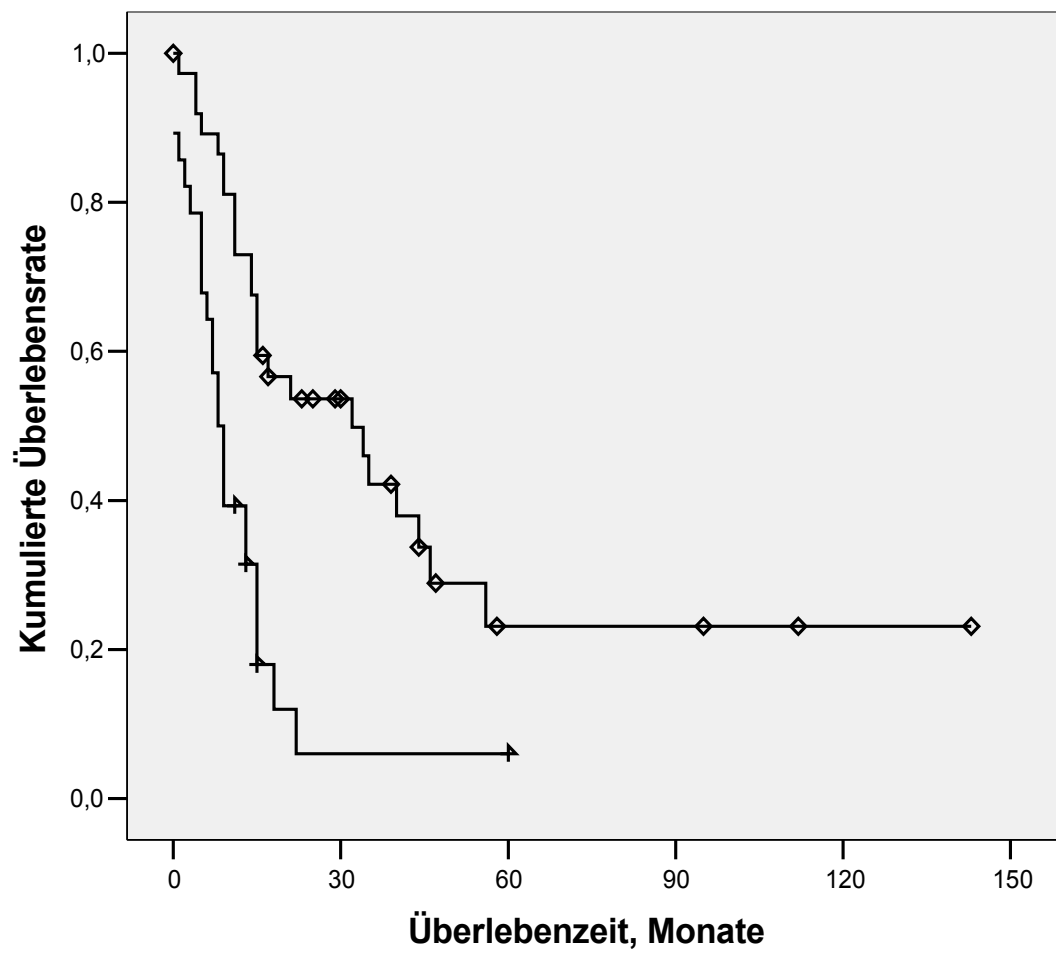

$\neg$ R1-Resektion

$\bigcap$ RO-Resektion

+ R1 zensiert

$\diamond \mathrm{RO}$ zensiert

Abbildung 23. Überlebensrate in Abhängigkeit von Resektionsart 


\subsubsection{Einfluss der Primär- bzw. Rezidiveingriffe}

Die mittlere Gesamtüberlebenszeit betrug nach primären Eingriffen 22 Monate, nach sekundären Eingriffen 21 Monate. Die folgende Kaplan-Meier-Kurve zeigt die etwas höhere Überlebensrate bei Primäreingriffen (die 5-Jahresüberlebensrate bei primären Eingriffen lag bei $18 \%$ vs. 15\% bei Rezidiveingriffen), wobei der Unterschied nicht statistisch signifikant ist (in Log-rank Test $\mathrm{p}=0,301$ ).

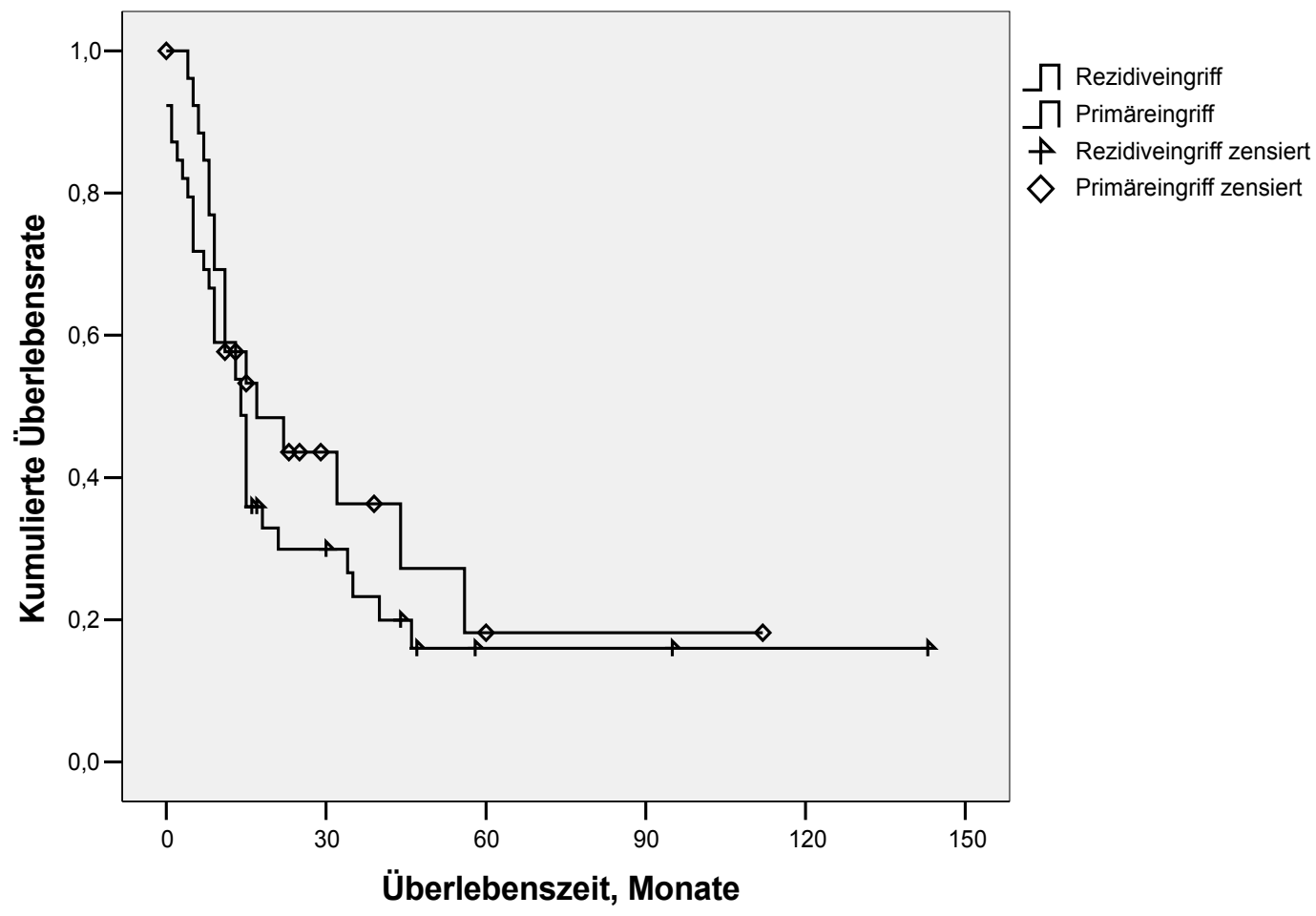

Abbildung 24. Überlebensrate bei Primär- und Rezidiveingriffen 


\subsubsection{Einfluss des Alters}

Die mittlere Gesamtüberlebenszeit lag in der Altersgruppe 30-60 Jahre bei 26 Monaten, in der Altersgruppe 61-90 Jahre bei 17 Monaten. Der Unterschied zwischen beiden Gruppen ist nicht statistisch signifikant (in Log-rank Test $p=0,152$ ).

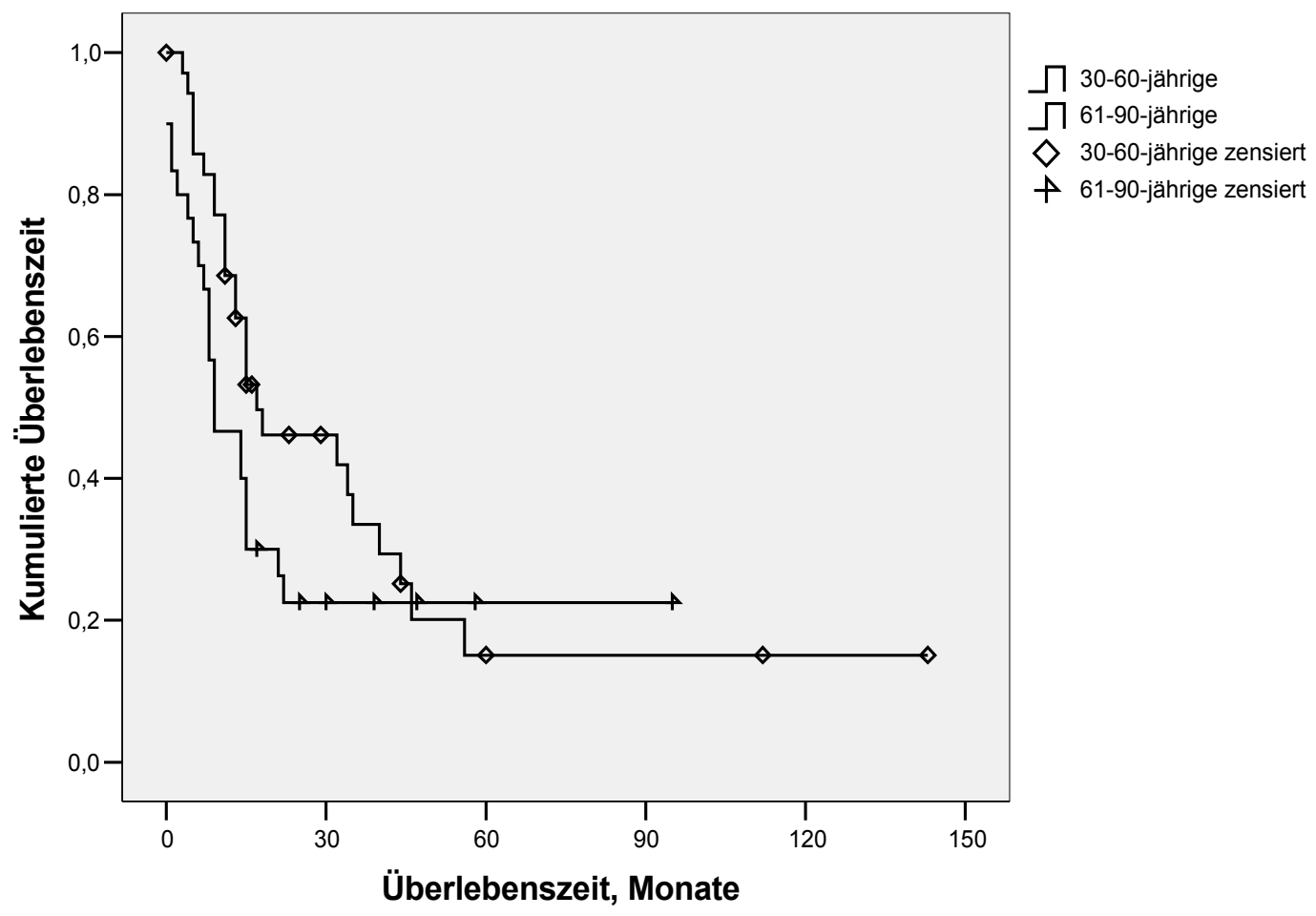

Abbildung 25. Überlebensrate in Abhängigkeit vom Patientinnenalter 


\subsubsection{Palliative Exenteration und die Überlebensrate}

Die mittlere Gesamtüberlebenszeit betrug nach Eingriffen mit einem kurativen Einsatz 24 Monate, bei palliativen Eingriffen 9 Monate. Die folgende Kaplan-Meier-Kurve zeigt die signifikant höhere Überlebensrate nach kurativer Exenteration gegenüber der palliativen Exenteration (in Log-rank Test $\mathrm{p}=0,034$ ).

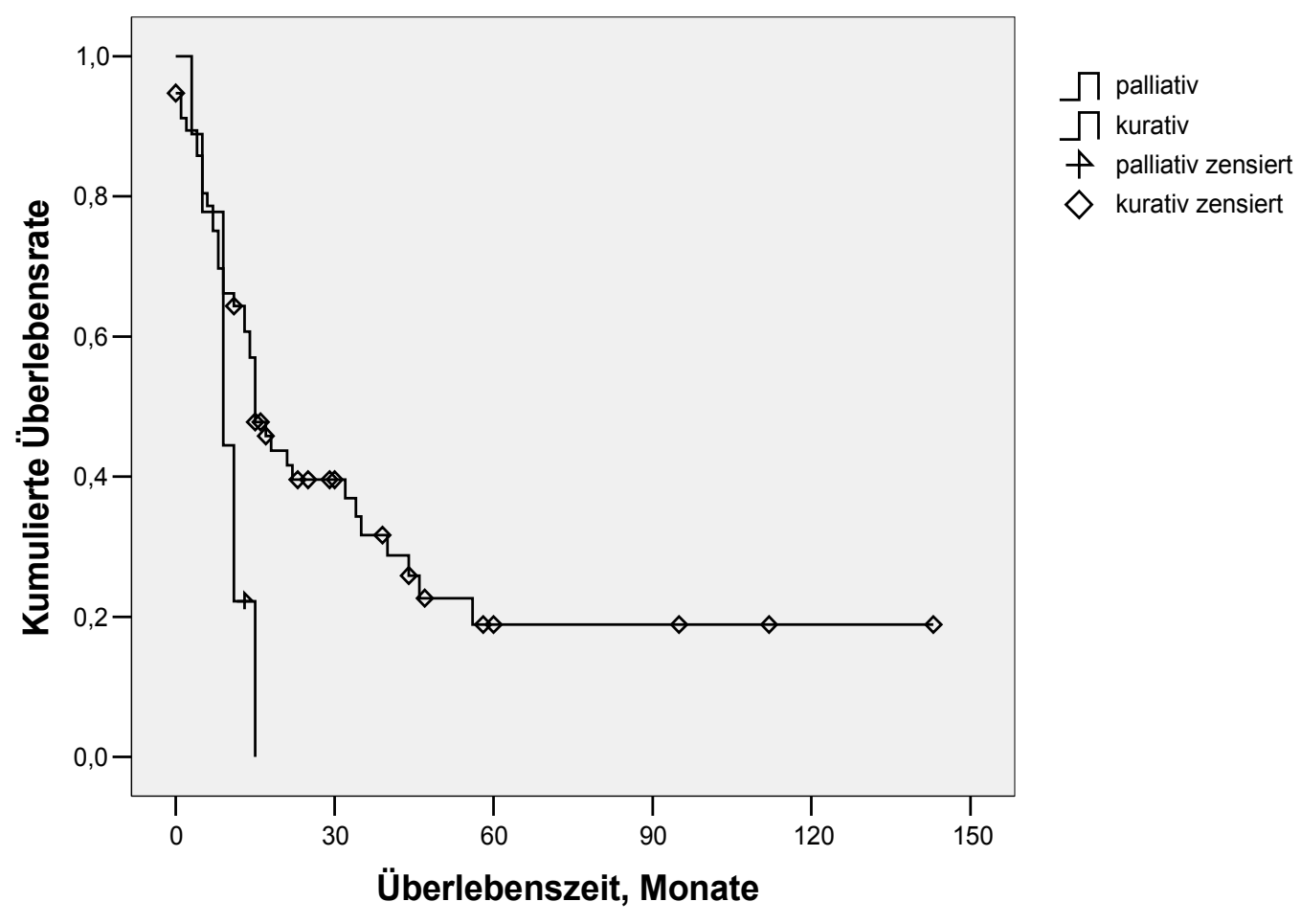

Abbildung 26. Überlebensrate bei palliativer Exenteration gegenüber der kurativen Exenteration 


\subsection{Einfluss verschiedner Parameter auf die progressionsfreie Überle- benszeit}

\subsubsection{Einfluss des Nodalstatus}

Die mittlere progressionsfreie Überlebenszeit bei einem negativen Nodalstatus (pN0) betrug 32 Monate. In der Gruppe der Patientinnen mit befallenen Lymphknoten (pN1) lag diese bei 11 Monaten und in der Gruppe der Patientinnen, bei denen keine Lymphonodektomie im Rahmen der Exenteration durchgeführt wurde ( $\mathrm{pNx}$ ) (entweder auf Grund der bereits durchgeführten Lymphonodektomie bei einem primären Eingriff oder im Rahmen einer palliativen Exenteration) bei 13 Monaten. Die folgende Kaplan-Meier-Kurve zeigt die signifikant höhere rezidivfreie Überlebensrate bei einem negativen Nodalstatus ( $38 \%$ bei $\mathrm{pN0}$ vs. $10 \%$ bei $\mathrm{pN} 1$ ) (in Log-rank Test $\mathrm{p}=0,037$ ).

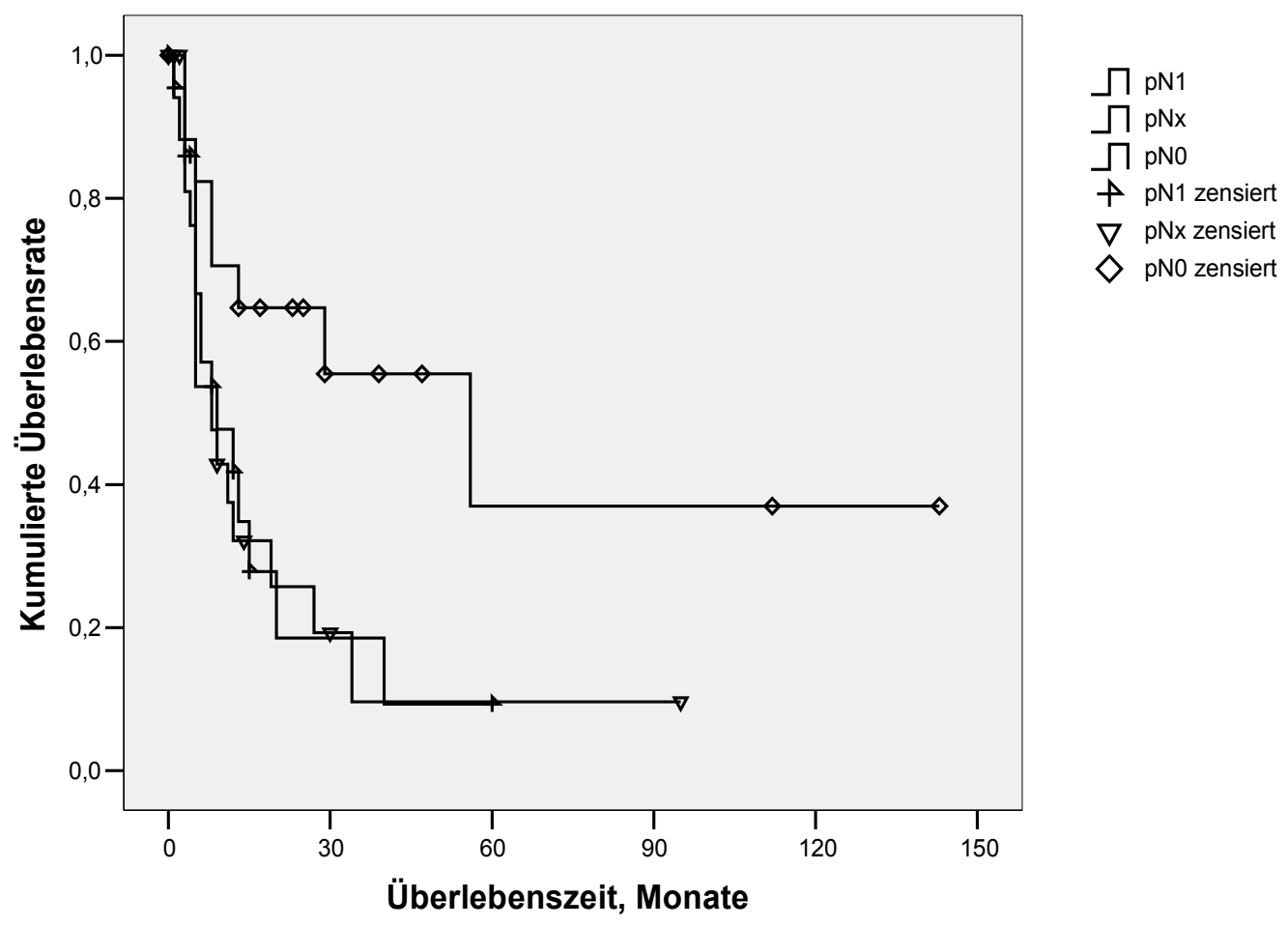

Abbildung 27. Rezidivfreie Überlebensrate in Abhängigkeit vom Nodalstatus 


\subsubsection{Einfluss der Resektionsart}

Die mittlere progressionsfreie Überlebenszeit in der Gruppe der Patientinnen, bei denen eine Resektion im Gesunden möglich war, betrug 25 Monate, bei einer R1-Resektion betrug die mittlere progressionsfreie Überlebenszeit 8 Monate. Die progressionsfreie 5-Jahresüberlebensrate lag bei einer R0-Resektion bei 22\% im Vergleich zu 14\% bei einer R1-Resektion (in Log-rank Test $\mathrm{p}=0,012$ ).

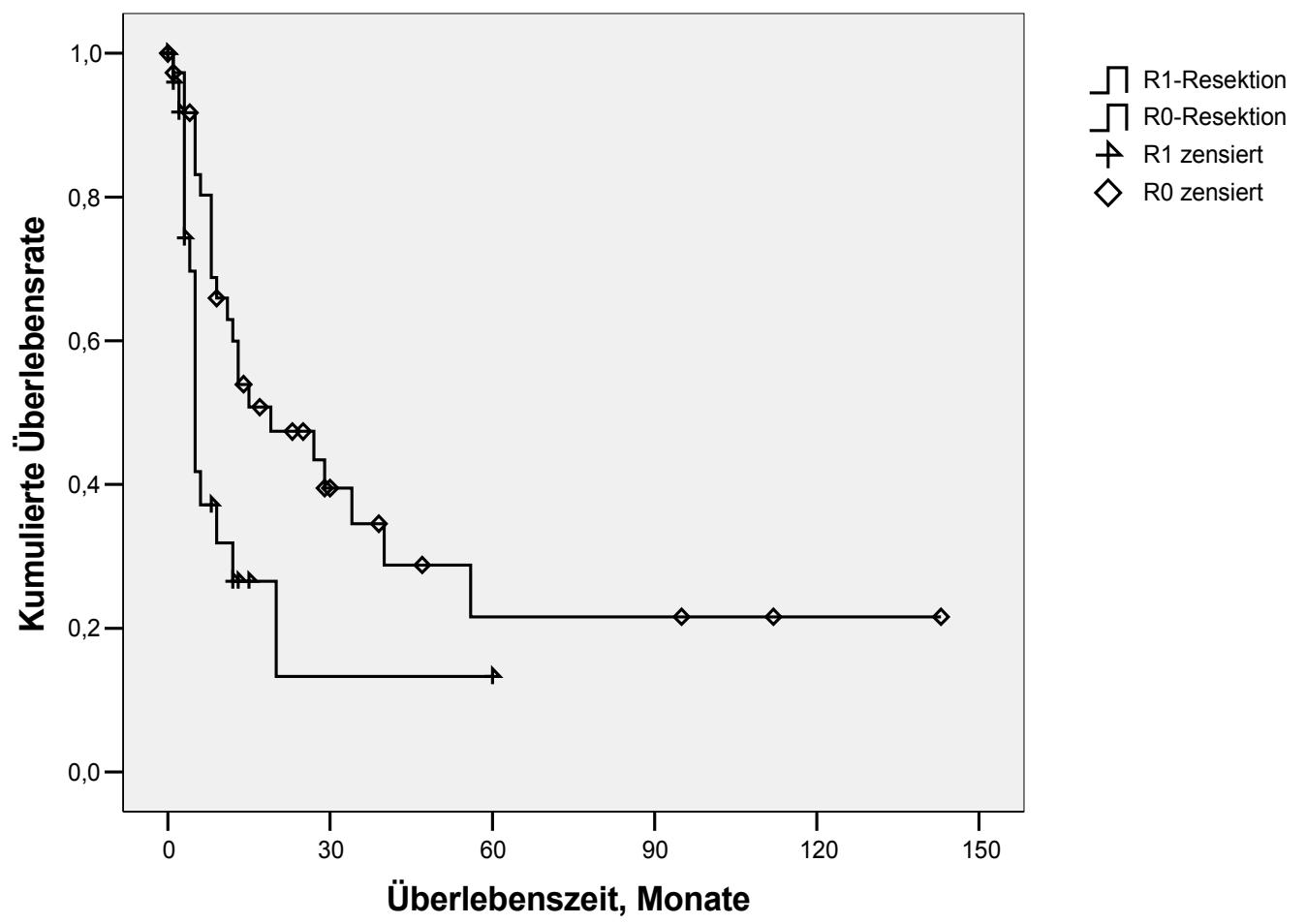

Abbildung 28. Rezidivfreie Überlebensrate in Abhängigkeit von Resektionsart 


\subsubsection{Einfluss der Primär- bzw. Rezidiveingriffe}

Die mittlere progressionsfreie Überlebenszeit betrug nach primären Eingriffen 18 Monate, nach sekundären Eingriffen 16 Monate. Die folgende Kaplan-Meier-Kurve zeigt die etwas höhere rezidivfreie Überlebensrate bei Primäreingriffen (25\% im Vergleich zu 15\% bei Rezidiveingriffen), wobei der Unterschied nicht statistisch signifikant ist (in Log-rank Test $p=0,449$ ).

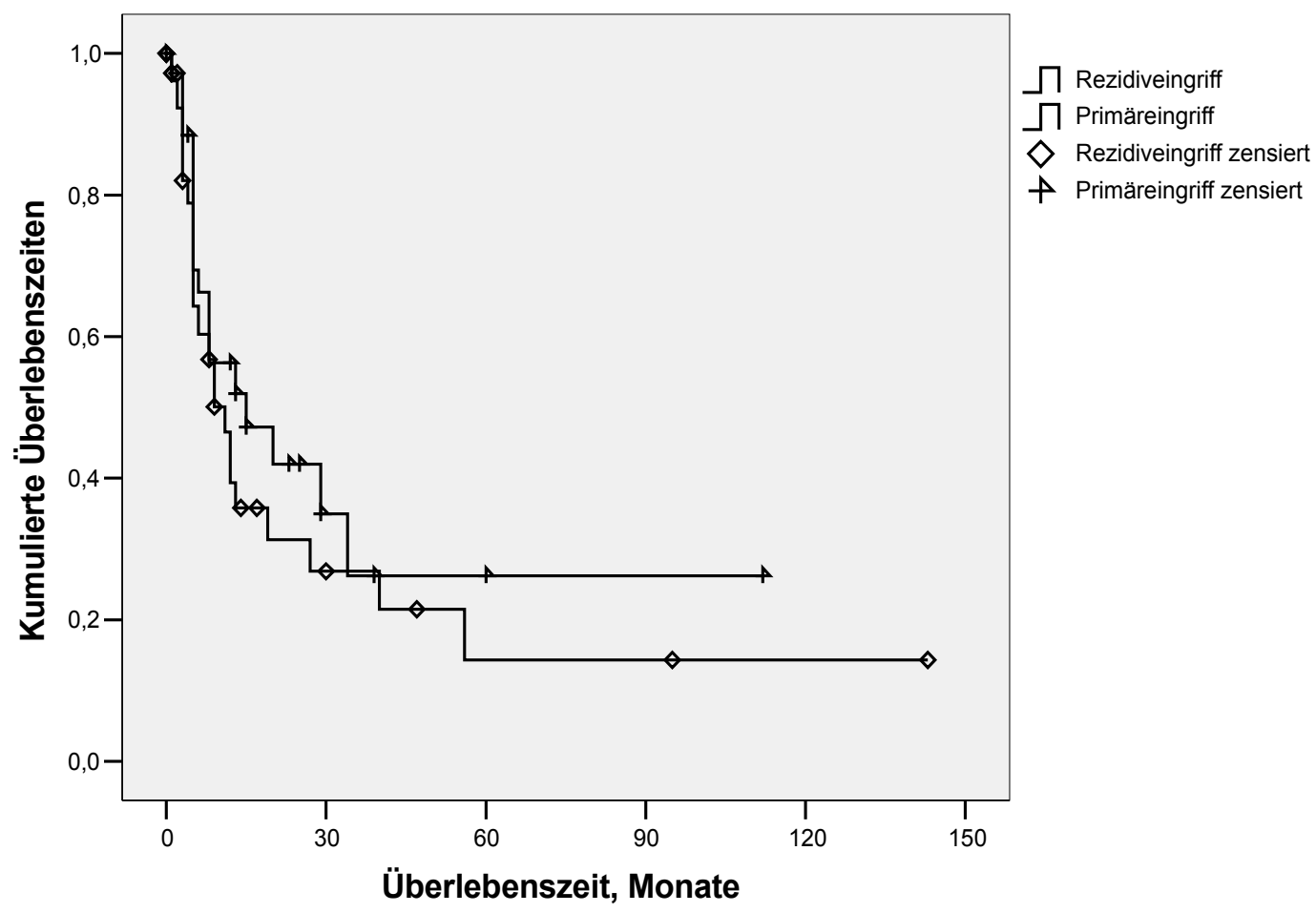

Abbildung 29. Rezidivfreie Überlebensrate bei Primär- und Rezidiveingriffen 


\subsubsection{Einfluss des Alters}

Die mittlere progressionsfreie Überlebenszeit betrug in der Altersgruppe 30-60 Jahre 20 Monate, in der Altersgruppe 61-90 Jahre 15 Monate. Die folgende Kaplan-Meier-Kurve zeigt etwas vergleichbare rezidivfreie Überlebensrate in beiden Gruppen (in der Gruppe der 30- bis 60-Jährigen lag die progressionsfreie 5-Jahresüberlebensrate bei $17 \%$ im Vergleich zu 19\% in der Gruppe der 61- bis 90-Jährigen), wobei der Unterschied nicht statistisch signifikant ist (in Log-rank Test $\mathrm{p}=0,732$ ).

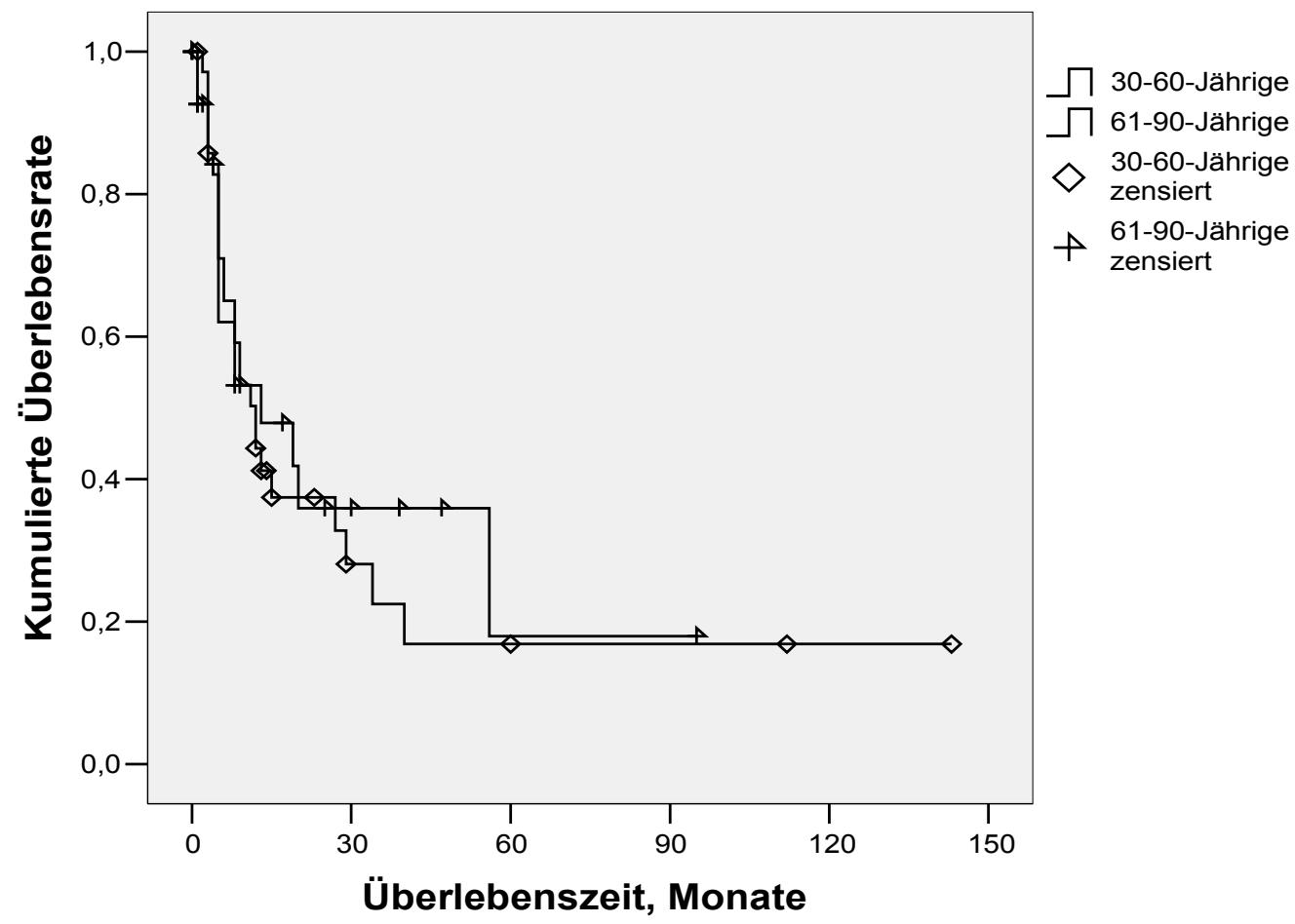

Abbildung 30. Rezidivfreie Überlebensrate in Abhängigkeit vom Patientinnenalter 


\subsection{2. Überlebenszeit bei verschiedenen Karzinomarten}

Das folgende Diagramm zeigt die mittlere Gesamt- und progressionsfreie Überlebenszeiten bei verschiedenen Karzinomarten.

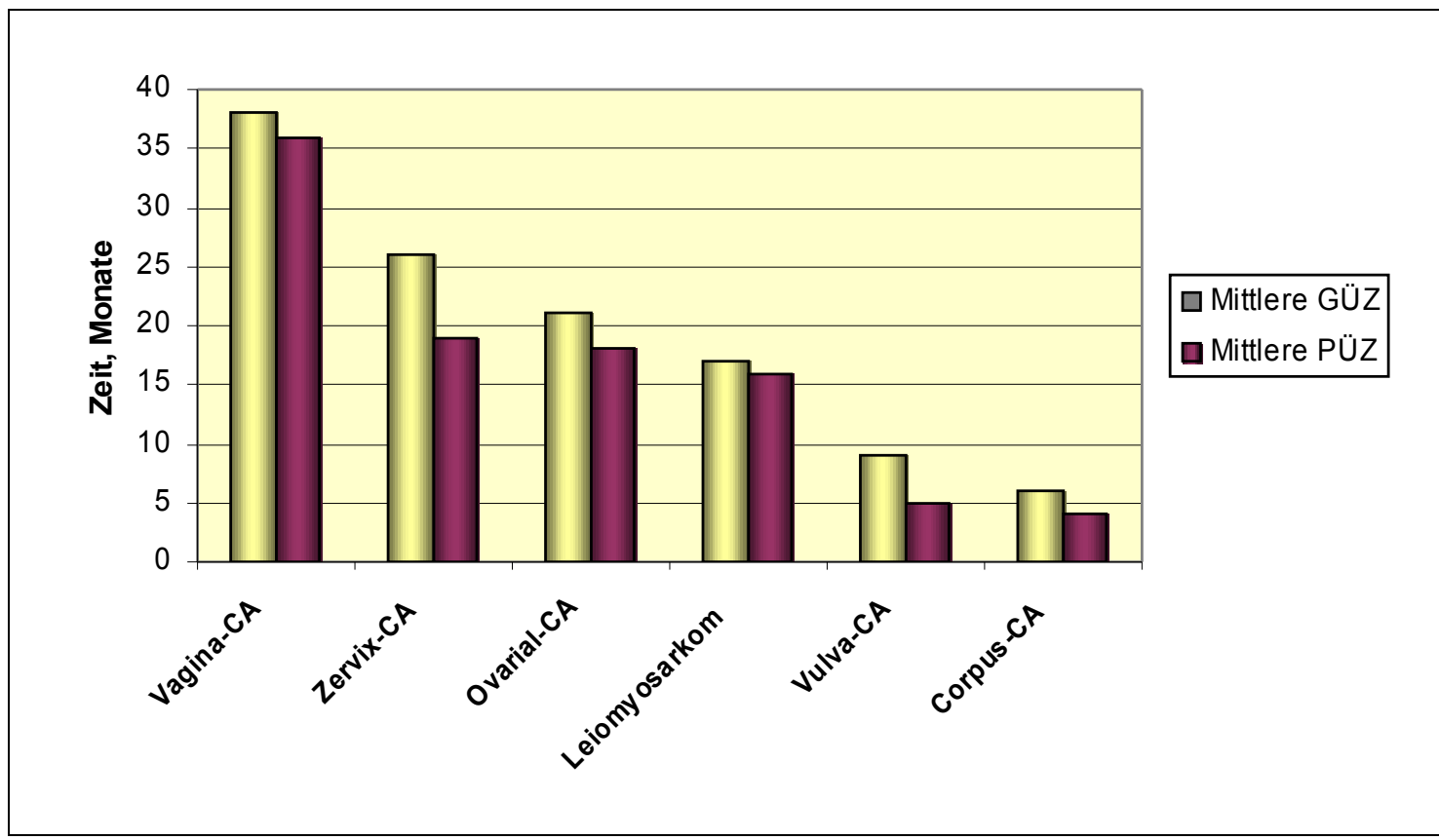

Abbildung 31. Mittlere Gesamt- (GÜZ) und progressionsfreie Überlebenszeiten (PÜZ) bei verschiedenen Karzinomarten 


\section{Diskussion}

Ziel dieser Arbeit war es, die Behandlungsergebnisse der an der Frauenklinik im Klinikum Kassel exenterierten Patientinnen retrospektiv zu analysieren und zu evaluieren. Rezidivfreies Überleben und Gesamtüberleben nach einer Exenteration sollten mit Daten internationaler und nationaler Studien verglichen und beurteilt werden. Zusätzlich wurden klinische und histopathologische Parameter hinsichtlich der Gesamtüberlebenszeit und der progressionsfreien Überlebenszeit untersucht.

Grundlage dieser Untersuchung war das Patientenkollektiv von 66 Frauen, die im Zeitraum von Januar 1991 bis Dezember 2005 an der Frauenklinik im Klinikum Kassel exenteriert wurden.

Das längste Beobachtungsintervall betrug 143 Monate, der mediane Beobachtungszeitraum betrug 22 Monate.

\subsection{Fallzahlen}

Im Zeitraum von 14 Jahren (1991 - 2005) wurden an der Frauenklinik im Klinikum Kassel 66 Exenterationen durchgeführt, d. h. ca. 5 Exenterationen pro Jahr, was den durchschnittlichen Fallzahlen an nationalen und internationalen Zentren entspricht (Tabelle 21). Auffallend ist die hohe Anzahl der exenterativen Eingriffe in den Anfangsjahren der radikalen Beckenchirurgie, die zu einem durch die deutlich höhere Inzidenz fortgeschrittener oder rezidivierter Karzinome, zum anderen durch das Fehlen chirurgischer Techniken zur Organerhaltung bedingt ist (Petri, 2001). In den letzten Jahren ist die Zahl der Exenterationen deutlich zurückgegangen, was durch verschiedene Faktoren, wie eine bessere Patientenselektion, Einführung der Vorsorgemaß- 
nahmen und damit verbundene Senkung der Zahl der invasiven Karzinome, Optimierung der Primäroperationen und eine deutlich gezieltere Strahlen- und Chemotherapie erklärt werden kann.

\subsection{Altersverteilung}

Bei unserer Auswertung waren die meisten Patientinnen zwischen 51-60 Jahre alt. Das Patientinnenalter variierte von 34 bis 87 Jahren. Das Durchschnittsalter lag bei 59,1 Jahren.

Vergleicht man unsere Ergebnisse mit den Daten aus der Literatur, so findet man eine vergleichbare Altersverteilung. Aleksic beschreibt in seiner Arbeit ein Durchschnittsalter von 59,8 Jahren (Aleksic et al., 1998). Eine ähnliche Altersverteilung eruierten in ihren Auswertungen Kolbert (60 Jahre (28 - 87 Jahre)), Hafner (60 Jahre (19 - 83)), Pinelo (55,7 Jahre), Fleisch (55 Jahre (22 - 77 Jahre)), Morley (53 Jahre (21 - 74 Jahre)) und Crozier (47 Jahre (22 - 70 Jahre)) (Crozier et al., 1995; Kolbert et al., 1997; Morley et al., 1989; Hafner et al., 1992; Pinelo et al., 2006; Fleisch et al., 2007). In der Studie von Berek reichte das Altersspektrum von 26 bis 74 Jahren (Berek et al., 2005). In der Arbeit von Durgatosh lag das Durchschnittsalter bei 45 Jahren (Durgatosh et al., 2004).

Das fortgeschrittene Alter stellt nach Meinung der meisten Autoren keine Kontraindikation zur Exenteration dar (Lambrou et al., 2005; Matthews et al., 1992; Hafner et al., 1992; Aleksic et al., 1998; Rodriguez-Bigas et al., 1996). Matthews et al. haben in ihrer Studie den postoperativen Verlauf von 63 Patientinnen untersucht, die zum Zeitpunkt der Exenteration 65 Jahre oder älter waren. Dabei konnte kein signifikanter Unterschied in den Mortalitäts- sowie Überlebensraten im Vergleich zur Patientinnengruppe, die jünger als 65 Jahre waren (Mortalitätsrate $11 \%$ vs. $8 \%, p=0,51$;

5-Jahresüberlebensrate $46 \%$ vs. $45 \%, p=0,52$ ) gezeigt werden (Matthews et al., 1992). 
In unserer Auswertung konnte eine etwas erhöhte postoperative Morbidität sowie niedrigere Überlebensraten bei älteren Patientinnen festgestellt werden, dieser Zusammenhang war allerdings statistisch nicht signifikant.

\subsection{Operationsdauer, Blutverlust}

In unserer Auswertung lag die durchschnittliche Operationsdauer bei 5,6 Stunden. Petri berichtet von einer durchschnittlichen Operationsdauer von 5,2 Stunden (170 - 660 min), in der Arbeit von Lorenz betrug diese durchschnittlich 11,4 Stunden (7 - 20 Stunden), in der Auswertung von Fleisch hat die Operation im Durchschnitt 8,1 Stunden gedauert (Petri, 2001; Lopez et al., 2004; Fleisch et al., 2007).

Es konnte eine signifikant längere OP-Dauer bei Rezidiveingriffen nachgewiesen werden, die durch eine erschwerte Präparation bei veränderten anatomischen Verhältnissen im voroperierten Gebiet erklärt werden kann.

Intraoperativ erhielten unsere Patientinnen im Median vier Erythrozytenkonzentrate. Fleisch beschreibt in seiner Arbeit eine perioperative Transfusion von 5,6 Erythrozytenkonzentraten (Fleisch et al., 2007). In der Literatur wird der Blutverlust bei den exenterativen Eingriffen mit 1000 bis 11000 ml angegeben (Lopez et al., 2004; Pearlman et al., 1994; Petri, 2001).

In unserer Arbeit konnte eine höhere Transfusionsmenge bei vorangegangener Bestrahlung oder Operation, sowie bei älteren Patientinnen gezeigt werden. Diese Tendenz war jedoch statistisch nicht signifikant. 


\subsection{Mortalität und Morbidität}

Die in der Literatur angegebenen Zahlen für Komplikationen nach pelviner Exenteration schwanken sehr und liegen zwischen $32 \%$ und $84 \%$ für Morbidität und zwischen $0 \%$ bis $14 \%$ für Mortalität (Tabelle 21 ).

Dank der Fortschritte in der Anästhesie und der perioperativen Intensivmedizin, der wesentlich besseren Patientenselektion durch präoperative Staging-Untersuchungen und der Entwicklung neuer operativer Techniken (Harnableitungen, Stapler) konnten die Mortalitätsraten vom $20-30 \%$ in den 50 er Jahren bis auf $5-10 \%$ in den 90 er Jahren gesenkt werden.

In unserem Patientinnenkollektiv gab es perioperativ insgesamt vier Todesfälle, somit beträgt die Mortalität 6\% und liegt auch im Vergleich zu anderen Publikationen in Anbetracht des Operationsausmaßes in einem vergleichbaren Bereich (Tabelle 21). Die Todesursachen waren eine massive intraoperative Blutung, sepsisbedingtes Multiorganversagen sowie eine Embolie aufgrund der generalisierten arteriellen Verschlusskrankheit. Rodriguez-Bigas et al. beschreiben als häufigste Todesursachen intraabdominale Abszedierung mit Peritonits, necrotisierende Fasziitis, Pneumonie und Blutung (Rodriguez-Bigas et al., 1996). Aleksic und Hafner geben Sepsis als häufigste Todesursache an (Hafner et al., 1992; Aleksic et al., 1998). In der Studie von Matthews et al. war das Multiorganversagen die häufigste Todesfallursache (Matthews et al., 1992).

Insgesamt traten in unserem operierten Patientinnenkollektiv in 61\% der Fälle postoperative Komplikationen auf, was mit den Daten in der Literatur vergleichbar ist (Tabelle 21). Am häufigsten werden in der Literatur urologische und gastrointestinale (vor allem Ileus und Anastomoseinsuffizienz) Komplikationen genannt (Hafner et al., 1992; Di Saia et al., 1973; Lopez et al., 2004; Shepherd et al., 1986). 
In unserem Patientinnengut traten am häufigsten gastrointestinale Komplikationen (Ileus und Anastomoseinsuffizienz) (18\%) und Wundheilungsstörungen (15\%) auf.

Verschiedene Faktoren, welche die Morbidität nach pelviner Exenteration beeinflussen, wurden in diversen Studien untersucht.

Die präoperative Radiatio wird in der Literatur als signifikantester Risikofaktor für das Auftreten von Komplikationen angesehen. Eine Strahlentherapie im kleinen Becken führt zu verschiedenen Reaktionen im Gewebe. Dabei kommt es zu einer Bindegewebsvermehrung (Fibrose), zu einem dauerhaften Verlust von funktionsfähigen Organzellen (Atrophie), zu einer Verödung der versorgenden kapillären Blutgefäße mit Erweiterung der vorangehenden kleinen Arterien und Venen (Teleangiektasien) sowie zu damit verbundenen Funktionseinbußen des Organs und Verhärtungen des Unterhautfettgewebes, des Bindegewebes und der Muskulatur (Kurzgefasste Interdisziplinäre Leitlinien, 2002). Die Präparation des bestrahlten fibrosierten Gewebes ist oft erschwert und mit einem ausgeprägten Blutverlust sowie einem höheren Verletzungsrisiko verbunden (Petri, 2001). Ein wesentlicher Faktor, der zur erhöhten Morbidität nach Radiatio beiträgt, ist die verzögerte Wundheilung in bestrahltem Gewebe, sowie die Neigung zur Fistelbildung. (Hafner et al., 1992)

Jakowatz et al. verglichen in ihrer Arbeit Komplikationsraten in Abhängigkeit von präoperativer Radiatio. Dabei betrug die Morbiditätsrate bei vorbestrahlten Patienten 67\%, in der Gruppe ohne vorangegangene Radiatio traten die Komplikationen bei $26 \%$ der Patienten auf (Jakowatz et al., 1985). Hafner et al. beschreiben eine Morbiditätsrate von $100 \%$ bei präoperativer Bestrahlung vs. $63 \%$ bei nicht vorbestrahlten Patienten (Hafner et al, 1992). In Serien von Houvenaeghel, Di Saia und Lopez konnte auch ein signifikanter Anstieg der postoperativen Komplikationen bei vorbestehender Radiatio gezeigt werden (Houvenaeghel et al., 2004; Di Saia et al., 1973; Lopez et al., 2004).

Vergleicht man die Komplikationsraten nach Primär- und Rezidiveingriffen, so zeigen 
Wydra et al. in ihrer Studie die höhere Morbidität bei Rezidiveingriffen (Wydra et al., 2006). Vermaas konnte hingegen in seiner Arbeit keinen signifikanten Unterschied in diesen Patientengruppen zeigen (Vermaas et al., 2007).

Bei der Auswertung anderer Faktoren sind die Studienergebnisse zum Teil sehr kontrovers.

So zeigen Jakowatz et al., dass die Exenterationsart, das Operationsausmaß, die Art der Harnableitung und die Tumorentität keinen Einfluss auf die Komplikationsraten haben (Jakowatz et al., 1985). In den Serien von Pearlman ist das Operationsausmaß ein signifikanter Risikofaktor für eine erhöhte Morbidität (Pearlman et al., 1994). Wydra zeigt in seiner Arbeit signifikant höhere Komplikationsraten bei einer Exenteration wegen eines Vulvakarzinoms, Houvenaeghel beschreibt die totale Exenteration als signifikanten Risikofaktor für erhöhte Morbidität (Wydra et al., 2006; Houvenaeghel et al., 2004). Karsenty zeigt, dass die kontinente Harnableitung mit einer höheren Morbidität verbunden ist; in der Arbeit von Houvenaeghel war hingegen die Rate der früh-postoperativen schwerwiegenden Komplikationen mit 4,8\% statistisch signifikant niedriger nach kontinenter Harnableitung als nach einer Conduitanlage (27,4\%). Goldberg et al. konnten hingegen keinen Unterschied in Komplikationsraten zwischen verschiedenen Harnableitungsformen nachweisen (Houvenaeghel et al., 2004; Karsenty et al., 2005; Goldberg et al., 2006).

In unserer Arbeit wurden die Komplikationsraten (nach Schweregrad) in Abhängigkeit von präoperativer Radiatio, Art des Eingriffs und der Harnableitung, sowie der Einfluss des Patientinnenalters untersucht. Dabei traten die Komplikationen (vor allem tödliche Komplikationen) nach einer vorangegangenen Bestrahlung oder Operation, bei einer inkontinenten Harnableitung und bei älteren Patientinnen häufiger auf, dieser Zusammenhang war jedoch wegen der geringen Fallzahl statistisch nicht signifikant. 


\subsection{Indikation}

In unserem Patientinnenkollektiv war der Eingriff bei Zervixkarzinomen am häufigsten (56,1\% der Fälle), gefolgt von Korpuskarzinomen (12,1\%) und Vaginal- und Ovarialkarzinomen (jeweils 9,1\%). Die anderen Tumorentitäten waren Vulvakarzinom, Sarkom und Tubenkarzinom.

Betrachtet man die Daten aus der Literatur, so zeigt die Verteilung der Tumorentitäten bei der OP-Indikation eine große Spannbreite. Der Anteil der Zervixkarzinome liegt bei 13 - 97\%, der der Vulvakarzinome bei 1 - 61\%, der der Korpuskarzinome bei 2-13\%, der der Vaginalkarzinome bei 2 - 14\%, der der Ovarialkarzinome und der der Sarkome bei 1 - 8\% (Brunschwig, 1959; Kiselow, 1967; Rodriguez-Bigas et al., 1996; Aleksic et al., 1998; Houvenaeghel et al., 2004; Durgatosh et al., 2004; Berek et al., 2005; Lambrou et al., 2005; Morley et al., 1989; Goldberg et al., 2006; Wydra et al., 2006; Pinelo et al., 2006; Mourton et al., 2006; Fleisch et al., 2007). Die meisten Autoren sehen allerdings ein lokal fortgeschrittenes oder ein rezidivierendes Zervixkarzinom, welches sich durch ein vorzugsweise lokales Wachstum und erst spätere Fernmetastasierung ausgezeichnet, als die häufigste Indikation zur Exenteration (Brunschwig, 1960; Kiselow, 1967; Di Saia et al., 1973; Rodriguez-Bigas et al., 1996; Petri, 2001; Hillemans et al., 2004; Lambrou et al., 2005).

Im Vergleich zu den vorgenannten Studien lag der Anteil der Exenterationen bei Ovarialkarzinomen in unserem Patientinnenkollektiv mit 9,1\% bezogen auf die Gesamtzahl der exenterativen Eingriffe relativ hoch. Bei Patientinnen mit Ovarialkarzinomen zeigen viele Autoren bei der Indikationsstellung zur Exenterationsoperation wegen der frühzeitigen intraperitonealen Metastasierung eine große Zurückhaltung (Petri, 2001; Lopez et al., 2005; Aleksic et al., 1998; Rodrigues-Bigas et al., 1996; Lambrou et al., 2005). Andere Studien dagegen vertreten ausgedehnte abdominalchirurgische Eingriffe sowohl bei einer Primäroperation als auch beim Auftreten eines späten platinsensiblen Rezidivs in gut selektierten Patientinnengruppen (guter Allgemeinzustand, Tumor- 
freiheit nach einer Primäroperation oder niedriges Tumorstadium bei Erstdiagnose, kein Nachweis von Aszites beim Rezidiv) (Schröer et al., 2006; Buttarelli et al., 2006, Harter et al., 2006). Buttarelli et al. untersuchten in einer retrospektiven Studie 44 Patientinnen mit einem fortgeschritten Ovarialkarzinom, die im Rahmen einer Primäroperation eine hintere Exenteration erhalten haben. Die Autoren zeigten eine mittlere Überlebenszeit von 36,6 Monaten, niedrige Raten an postoperativen Komplikationen und gute postoperative Lebensqualität (Buttarelli et al., 2006).

In unserem Patientinnenkollektiv wurde in sechs Fällen eine Exenteration bei einem Ovarialkarzinom durchgeführt. Dabei handelt es sich um fünf Eingriffen bei Spätrezidiven im kleinen Becken nach einer Primäroperation und einer platinhaltigen Chemotherapie. Einer Patientin wurde der Eingriff als eine Primäroperation vorgenommen. Eine Patientin aus dieser Gruppe erhielt die Exenteration in einer Palliativsituation wegen massiver Blasenblutungen und rezidivierender Blasentamponaden.

Betrachtet man den onkologischen Verlauf, beträgt die mittlere progressionsfreie Zeit 18 Monate (17 Monate im Gesamtkollektiv) und die mittlere Überlebenszeit 21 Monate (22 Monate im Gesamtkollektiv), was den Einsatz der Exenteration bei Ovarialkarzinomen bei entsprechender Indikation rechtfertigt.

In unserem Patientenkollektiv wurde in 13,6\% der Fälle eine palliative Exenteration durchgeführt. Es konnte kein signifikanter Unterschied hinsichtlich der Komplikationsrate, der Dauer des Krankenhausaufenthaltes und der intraoperativen Transfusionsmenge bei einem palliativen gegenüber einem kurativen Eingriff gezeigt werden. Die Überlebenszeiten mit einem mittleren Wert von zehn Monaten waren allerdings bei palliativen Exenterationen signifikant niedriger als bei kurativen Eingriffen.

Eine palliative Exenteration wird in der Literatur unterschiedlich betrachtet. Die Exenteration als palliativer Eingriff wird von vielen Autoren, vor allem aus dem angloamerikanischen Raum abgelehnt. Als Gründe dafür werden geringere Heilungschancen bei Tumoren, die sich über das kleine Becken hinaus ausdehnen, sowie hohe Morbidität und operative Mortalität genannt (Anthapoulos et al., 1989; Lawhead et al., 1989; Soper 
et al., 1989; Lopez et al., 2004). Andere Autoren rechtfertigen den palliativen Einsatz. Magrina zeigt in seiner Arbeit, dass die perioperative Komplikationsrate bei einem palliativen Eingriff vergleichbar ist mit der bei einem kurativen Eingriff (Magrina et al., 1997). Stanhope und Symmonds untersuchten in einer retrospektiven Studie 59 Patienten, die einer palliativen Exenteration unterzogen wurden. Die Autoren zeigten eine mediane Überlebenszeit von 19 Monaten und eine 2-Jahrenüberlebensrate von $46 \%$ (Stanhope, Symmonds, 1985).

Lambrou et al. definierten Patientengruppen, die von einer palliativen Exenteration profitieren können: Patienten mit kleinen Fernmetastasen und einem operablen symptomatischen Beckenrezidiv, Patienten mit rezidivierenden Infekten, unheilbaren Fisteln und/oder ausgedehnten Strahlenfibrosen, mit transfusionsbedürftigen Tumorblutungen oder therapieresistenten Schmerzen (Lambrou et al., 2005). Brophy et al. berichten nach Auswertung von Kriterien wie Anzahl von Arztbesuchen, Ernährungsstatus, Schmerzangabe und Medikamenteneinnahme, dass die Lebensqualität bei $88 \%$ der Patienten $(\mathrm{n}=35)$, die einer palliativen, symptomorientierten Operation unterzogen worden waren, deutlich verbessert werden konnte (Brophy et al., 1994).

\subsection{Gesamt- und progressionsfreie Überlebensraten}

Die Heilungsquoten nach Exenteration variieren in der Literatur erheblich. Die

5-Jahres-Überlebensraten liegen zwischen 20 und 80\% (Tabelle 21). Die großen Differenzen in den Angaben können durch die unterschiedliche Indikationsstellung erklärt werden. Die guten Überlebensraten in den Anfangsjahren der Exenterationschirurgie bei sehr großen Patientenzahlen trotz deutlich höherer Morbidität, können durch eine wesentlich großzügigere Indikation zur Exenteration erklärt werden (z.B. bei Frauen, bei denen heutzutage bereits bei beginnender Infiltration der Blasenwand oder des Rektosigmoids organerhaltend operiert wird, wurde damals oft eine Indikation zur 
vollständigen Exenteration gestellt) (Petri, 2001). Hohe Heilungsquoten bei angloamerikanischen Autoren können auf einer extrem strengen Patientenauswahl und grundsätzlicher Ablehnung der Palliativeingriffe beruhen.

In unserem Kollektiv lag die mittlere Gesamtüberlebenszeit bei 22 Monaten, die 5-Jahres-Überlebensrate betrug 34\%, was im Vergleich zu den Daten aus der Literatur etwas unter dem Durchschnitt liegt. Die Ursache kann zum einen mit dem vergleichsweise hohen Anteil der Palliativexenterationen in unserer Klinik erklärt werden (13,6\% der Gesamtoperationszahl), zum anderen auch mit dem hohen Anteil älterer Patientinnen (24\% unserer Patientinnen sind älter als 70 Jahre), die zum Teil eine höhere Komorbidität aufweisen.

Die Rezidivrate nach Exenteration wird in der Literatur mit 20-60\% angegeben, wobei oft nicht zwischen einem Lokalrezidiv und einer Fernmetastasierung unterschieden wird. (Aleksic et al., 1998; Lopez et al., 2004; Sharma et al., 2005; Pinelo et al., 2006; Pearlman, 1994). Die mittlere progressionsfreie Überlebenszeit variiert in den zitierten Arbeiten zwischen 7 und 17 Monaten, die 5-Jahres-progressionsfreie Überlebensrate liegt zwischen 32 und 80\%. In unserem Patientinnenkollektiv lag die Lokalrezidivrate bei 38\%, eine Fernmetastasierung trat in 18\% der Fälle auf. Somit betrug die Gesamtrezidivrate 56\%. Die mittlere progressionsfreie Überlebenszeit betrug 17 Monate.

Prognostische Faktoren, die das postoperative gesamt- und progressionsfreie Überleben der Patienten nach Exenteration beeinflussen können, sind Gegenstand verschiedener Studien und werden im Folgenden dargestellt. 


\subsubsection{Gesamtüberlebensraten}

Die meisten Autoren beschreiben den negativen Nodalstatus, eine R0-Resektion und einen kurativen Therapieansatz als wichtigste prognostische Faktoren für die Gesamtüberlebenszeit (Morley et al., 1989; Vermaas et al., 2007; Teran-Porcayo et al., 2006; Nguyen et al., 2005; Lopez et al., 2004; Aleksic et al., 1998; Durgatosh et al., 2004; Koehler et al., 2006; Fleisch et al., 2007). Lopez et al. definieren in ihrer Arbeit den Lymphknotenbefall auf Grund der deutlich schlechteren Prognose als relative Kontraindikation zur Exenteration.

Ketcham berichtet in seiner Arbeit von insgesamt 200 exenterierten Patienten. Nur ein Patient mit einem positiven Nodalstatus lebte nach 5 Jahren (Ketcham et al., 1970). In der Serie von Barber haben nur 5 von insgesamt 148 Patienten mit befallenen Lymphknoten 5 Jahre überlebt (Barber et al., 1988). Shoup et al. zeigten in ihrer Serie, dass die Überlebensraten bei den Patienten mit einer R0-Resektion doppelt so hoch waren wie bei denjenigen mit einer R1- oder R2-Resektion (Shoup et al., 2002). De Wilt et al. konnten hingegen bei der Untersuchung des onkologischen Verlaufs der 42 exenterierten Patientinnen keinen Einfluss des Lymphknotenbefalls und des verbliebenen Tumorrestes auf die gesamt- und progressionsfreie Überlebenszeiten zeigen (de Wilt et al., 2006). Houvenaeghel konnte nur bei einem paraaortalen Lymphknotenbefall eine Prognoseverschlechterung zeigen (Houvenaeghel et al., 2006).

Lopez et al. beschreiben folgende Faktoren, die eine angestrebte Resektion in sano erschweren können: ein Rezidiveingriff, Lymphangiosis carcinomatosa, fortgeschrittenes Tumorstadium, fortgeschrittenes Patientenalter (Lopez et al., 2005). Lampe et al. zeigen in einer Studie an 63 Patientinnen deutlich schlechtere Detektionsraten bei der Beurteilung der Resektionsränder (sowohl präoperativ bildgebend als auch intraoperativ) bei vorbestrahlten Patientinnen (Lampe et al., 2005). 
Die folgende Tabelle zeigt die Überlebensraten in Abhängigkeit von der Resektionsart.

\begin{tabular}{|l|c|c|c|}
\hline \multicolumn{1}{|c|}{ Autor } & $\mathrm{N}$ & ÜR bei R0-Resektion & ÜR bei R1/2-Resektion \\
\hline Oliveira et al. & 96 & Mediane ÜZ 40 Monate & Mediane ÜZ 21 Mo \\
\hline Ike et al. & 45 & 5-JÜZ 31\% & 5-JÜZ 7\% bei R1 und 0\% \\
& & & bei R2 \\
\hline Wiig et al. & 47 & 5-JÜZ 36\% & 5-JÜZ 18\% \\
\hline Yamada et al. & 64 & 5-JÜZ 23\% & 5-JÜZ 0\% \\
\hline Koehler & 55 & 5-JÜZ 55,2\% & 2-JÜZ 10,2\% \\
\hline Klinikum Kassel & 66 & 5-JÜZ 22\% & 5-JÜZ 7\% \\
\hline
\end{tabular}

Tabelle 19. Überlebensraten in Abhängigkeit von der Resektionsart

Die folgende Tabelle zeigt die 5-Jahres-Überlebensraten in Abhängigkeit vom Nodalstatus (Petri, 2001).

\begin{tabular}{|l|c|c|c|}
\hline \multicolumn{1}{|c|}{ Autor } & $\mathrm{N}$ & $\mathrm{N} 0-$ Status & N1-Status \\
\hline Kiselow & 88 & $50 \%$ & $21,4 \%$ \\
\hline Ketcham & 200 & $33 \%$ & $11 \%$ \\
\hline Barber & 166 & $17,4 \%$ & $5,1 \%$ \\
\hline Symmonds & 68 & $42 \%$ & $15 \%$ \\
\hline Friedberg & 115 & $63,3 \%$ & $12,5 \%$ \\
\hline Klinikum Kassel & 66 & $51 \%$ & $8 \%$ \\
\hline
\end{tabular}

Tabelle 20. Überlebensraten in Abhängigkeit vom Nodalstatus 
$\mathrm{Zu}$ den anderen in der Literatur untersuchten und kontrovers diskutierten prognostischen Faktoren gehören Alter, Tumorstadium, Art der Exenteration sowie die Art der Blasen- und Darmrekonstruktion. In Arbeiten von Hatch, Morley und von Sharma konnte kein Einfluss der Art der Exenteration und der Rekonstruktion auf die Gesamtüberlebensraten bestätigt werden (Hatch et al., 1988; Morley et al., 1989; Sharma et al., 2005). Houvenaeghel und Koehler hingegen werten die Art des Eingriffes als einen signifikanten Prognosefaktor für das Gesamtüberleben (Houvenaeghel et al., 2006; Koehler et al., 2006). So betrug die Gesamtüberlebensrate in der Auswertung von Koehler bei den Primäreingriffen 52,5\% und in der Rezidivgruppe 26,7\% ( $p=0,0472$ ). Jimenez et al. konnten in ihrer Arbeit auch die signifikant höheren Überlebensraten bei Primäreingriffen nachweisen. Die Autoren erklären diese Tendenz durch höhere Raten an R1- und R2-Resektionen bei voroperierten Patienten (Jimenez et al., 2003).

Hatch, de Wilt, Morley und Koehler zeigen, dass das Tumorstadium keinen Einfluss auf das Gesamtüberleben hat. De Wilt zeigt zusätzlich, dass Patientenalter und Rezidiveingriffe keine Prognosefaktoren für das Gesamtüberleben sind. Koehler sieht keinen Einfluss des Alters und des Typs der Exenteration auf das Langzeitüberleben (Hatch et al., 1988; de Wilt et al., 2006; Koehler et al., 2006; Morley et al., 1989).

In unserer Arbeit wurde die Überlebensrate in Abhängigkeit vom Nodalstatus, Alter, der Resektionsart, der Art des Eingriffs untersucht.

Es konnte die signifikant höhere Überlebensrate bei negativem Nodalstatus, R0-Resektion und bei einem kurativen Therapieansatz nachgewiesen werden. Eine prognostische Bedeutung des Alters und der Art der Exenteration (Primär- vs. Rezidiveingriff) konnte dagegen nicht gezeigt werden.

Vergleicht man die Raten an R0-Resektionen in verschiedenen Patientinnengruppen, so zeigten sich niedrigere R0-Resektionsraten bei vorbestrahlten Patientinnen, in der Gruppe der älteren Patientinnen sowie bei palliativen Exenterationen, wobei dieser Zusammenhang in den zwei erstgenannten Gruppen statistisch nicht signifikant war. Diese Tendenz kann durch typische Veränderungen des Gewebes unter Strahlenthera- 
pie und dadurch erschwerte Unterscheidung zwischen einer Strahlenfibrose und dem Tumorgewebe, sowie durch eine höhere Prävalenz der Rezidiveingriffen, der vorangegangen systemischen Therapie sowie der palliativen Eingriffe in der Gruppe der älteren Patientinnen erklärt werden.

\subsubsection{Progressionsfreie Überlebensraten}

Ein negativer Nodalstatus und Resektion in sano gehören nach Auffassung vieler Autoren zu den entscheidenden Faktoren, welche das progressionsfreie Überleben beeinflussen (Houvenaeghel et al., 2004; Kolbert et al., 1997; Vermaas et al., 2007; Mourton et al., 2007). Mourton et al. haben gezeigt, dass es bei einem positiven Nodalstatus im Durchschnitt in 2,4 Monaten postoperativ zur Progression der Erkrankung kam, bei einem negativen Lymphknotenstatus lag die progressionsfreie Überlebenszeit bei 7,3 Monaten $(p=0,005)$. Kolbert und Vermaas beschreiben niedrigere progressionsfreie Überlebensraten nach einer R1/R2-Resektion. De Wilt hingegen bestreitet den Einfluss der Resektionsart auf die progressionsfreie Überlebensrate (de Wilt et al., 2006). De Wilt et al. haben in ihrer Studie 42 Patientinnen untersucht, dabei konnte kein Zusammenhang zwischen dem Alter, der Exenterationsart, dem histologischen Befund, der Tumorlokalisation und dem Auftreten eines Rezidivs gezeigt werden. Sharma et al demonstrieren in ihrer Arbeit, dass der Exenterationstyp sowie die Art der Harn- und Stuhlableitung keinen Einfluss auf die progressionsfreie Überlebenszeit haben (Sharma et al., 2005).

In unserem Patientinnenkollektiv war die progressionsfreie Überlebensrate bei einem negativen Lymphknotenstatus und einer Resektion in sano signifikant höher, dabei spielten Alter und Art des Eingriffes (Primär- vs. Rezidiveingriff) keine signifikante Rolle. 
Diskussion

\begin{tabular}{|c|c|c|c|c|c|}
\hline & Fallzahl & $\begin{array}{c}\text { Anzahl der } \\
\text { Eingriffe } \\
\text { pro Jahr }\end{array}$ & $\begin{array}{l}\text { Morbidi- } \\
\text { tät, \% }\end{array}$ & $\begin{array}{l}\text { Mortalität, } \\
\%\end{array}$ & $\begin{array}{l}\text { 5-Jahres-ÜZ, } \\
\text { bzw. mittlere ÜZ }\end{array}$ \\
\hline Brunschwig (1947-1959) & 592 & 49 & k.A. & $23 \%$ & $33 \%$ \\
\hline Bricker (1950-1965) & 312 & 21 & $42 \%$ & $10 \%$ & $35 \%$ \\
\hline Aleksic (1988-1996) & 18 & 2 & $40 \%$ & $11 \%$ & Mittlere ÜZ 30 Mo. \\
\hline Anthapoulos (1982-1989) & 20 & 3 & $74 \%$ & $5 \%$ & $58 \%$ \\
\hline Berek (1956-2001) & 150 & 3 & k.A. & k.A. & $54-62 \%$ \\
\hline Buttarelli (1995-2003) & 44 & 6 & $13,6 \%$ & k.A. & $\begin{array}{l}\text { Mittlere ÜZ } \\
\text { 36,6 Mo. }\end{array}$ \\
\hline De Wilt (1991-2004)) & 42 & 3 & $75 \%$ & k.A. & $52 \%$ \\
\hline Durgatosh (1981-2000) & 42 & 2 & $62,5 \%$ & $10,4 \%$ & $77,6 \%$ \\
\hline Fleisch (1983-2002) & 203 & 11 & k.A. & $1 \%$ & $21 \%$ \\
\hline Goldberg (1987-2003) & 103 & 7 & k.A. & k.A. & $47 \%$ \\
\hline Hatch (1979-1989) & 31 & 3 & $32 \%$ & $0 \%$ & $68 \%$ \\
\hline $\begin{array}{l}\text { Houvenaeghel(1980-200 } \\
\text { 2) }\end{array}$ & 232 & 11 & $52 \%$ & $8 \%$ & $57,7 \%(10 \mathrm{~J})$ \\
\hline Karsenty (1993-2001) & 60 & 3 & $46 \%$ & $4,9 \%$ & k.A. \\
\hline Koehler (1998-2004) & 55 & 9 & $56,9 \%$ & $5,5 \%$ & $36,8 \%$ \\
\hline Kolbert (1983-1996) & 33 & 3 & $85 \%$ & $3 \%$ & Mittlere ÜZ 17 Mo. \\
\hline Kraybill (1966-1986) & 99 & 5 & $71 \%$ & $14 \%$ & $45 \%$ \\
\hline Lawhead (1979-1989) & 65 & 7 & k.A. & $9 \%$ & $23 \%$ \\
\hline Lopez (1979-2001) & 34 & 2 & $67,6 \%$ & $0 \%$ & $44 \%$ \\
\hline Morley (1964-1984) & 100 & 5 & $49 \%$ & $2 \%$ & $61 \%$ \\
\hline Mourton (1992-2003) & 37 & 3 & k.A. & k.A. & k.A. \\
\hline Nguyen (1992-2003) & 76 & 7 & $28 \%$ & $0 \%$ & $53 \%$ \\
\hline Pinelo (1995-2005) & 21 & 2 & k.A. & k.A. & Mediane ÜZ $29 \mathrm{Mo}$ \\
\hline Sharma (1980-1999) & 48 & 3 & $75 \%$ & $4,2 \%$ & Mediane ÜZ $35 \mathrm{Mo}$ \\
\hline $\begin{array}{l}\text { Teran-Porcayo } \\
(1984-1989)\end{array}$ & 42 & 8 & $65,3 \%$ & $4,8 \%$ & $65,8 \%$ \\
\hline Vermaas (1994-2004) & 35 & 4 & $50 \%$ & $3 \%$ & $52 \%$ \\
\hline Wydra (1996-2004) & 23 & 3 & k.A. & k.A. & $\begin{array}{l}\text { Mediane ÜZ } \\
\text { 6-74 Mo }\end{array}$ \\
\hline $\begin{array}{l}\text { Klinikum Kassel } \\
(1991-2005)\end{array}$ & 66 & 5 & $60,6 \%$ & $6 \%$ & Mittlere ÜZ 22 Mo \\
\hline
\end{tabular}

Tabelle 21. Literaturübersicht der Behandlungsergebnisse nach Exenteration 


\section{Zusammenfassung}

Die vorliegende Arbeit wertet die Behandlungsergebnisse der exenterativen Eingriffe aus, die an der Frauenklinik im Klinikum Kassel zwischen 1991 und 2005 durchgeführt worden sind. In diesem Zeitraum wurden die Daten von 66 Patientinnen erfasst, diese wurden bezüglich des stationären und poststationären Verlaufs analysiert. Zusätzlich wurden klinische und histopathologische Parameter hinsichtlich der Gesamtüberlebenszeit und der progressionsfreien Überlebenszeit untersucht.

Der Altermittelwert lag bei 59 Jahren.

Es wurden insgesamt 33 vordere Exenterationen, 24 totale und neun hintere Exenterationen durchgeführt. Zervixkarzinome stellten mit 57,6\% die häufigste Indikation zur Exenteration dar. In 27 Fällen wurde die Exenteration als Primäreingriff durchgeführt, bei 39 Patientinnen handelte es sich um einen Rezidiveingriff. In neun Fällen wurde die Exenteration als palliativer Eingriff durchgeführt.

Bei 37 Patientinnen (56,1\%) wurde der Tumor in sano reseziert. Bei 19 exenterierten Patientinnen (45\%) konnte ein Lymphknotenbefall nachgewiesen werden.

Histologisch konnte bei 41 Patientinnen (62\%) eine Blaseninfiltration nachgewiesen werden, ein Darmbefall zeigte sich in 20 Fällen (30,3\%).

Bei der Darmrekonstruktion handelte es sich in 15\% der Fälle um kontinenzerhaltende Anastomosen, bei 85\% der Patientinnen wurde ein Anus praeter naturalis angelegt. Bei der Harnableitung haben $76 \%$ der Patientinnen ein Ileum-Conduit erhalten, bei $24 \%$ der Patientinnen erfolgte eine kontinente Harnableitung.

Es kam bei insgesamt 40 Patientinnen $(60,6 \%)$ zu postoperativen Komplikationen. Die Komplikationen (vor allem tödliche Komplikationen) traten nach einer vorangegangenen Bestrahlung oder Operation, bei einer inkontinenten Harnableitung und bei älteren Patientinnen häufiger auf, dieser Zusammenhang war jedoch wegen der geringen Fallzahl statistisch nicht signifikant. 
Die Mortalitätsrate betrug 6\%.

Die mittlere Gesamtüberlebenszeit (ab dem Zeitpunkt der Operation) betrug 22 Monate. Die 5-Jahres-Überlebensrate betrug 34\%.

Bei 37 Patientinnen kam es im Laufe der Beobachtung zur Progression der Erkrankung. Diese äußerte sich in 25 Fällen durch Entstehung eines Lokalrezidivs, 12 Patientinnen entwickelten Fernmetastasen. Somit lag die Rezidivrate bei $56 \%$.

Als signifikante Einflussgrößen im Hinblick auf die Gesamtüberlebenszeit erwiesen sich in unserem Kollektiv ein negativer Nodalstatus, Resektion in sano und ein kurativer operativer Einsatz.

In Anbetracht der Angaben aus der Literatur sowie der Daten aus unserem Kollektiv stellt die pelvine Exenteration eine angemessene Alternative bei der Behandlung von lokal fortgeschrittenen oder rezidivierenden Tumoren im kleinen Becken dar. Patientinnen in einem guten Allgemeinzustand, ohne vorangegangene Bestrahlung, bei einem negativen Nodalstatus und einer Resektion in sano profitieren am meisten von einer Exenteration. Das exenterative Vorgehen ist auch bei Ovarialkarzinomen bei entsprechender Indikationsstellung gerechtfertigt. Bei außerordentlich belastenden regionären Tumorsymptomen ist eine Exenteration auch als palliativer Eingriff vertretbar.

Die sorgfältige Patientinnenselektion sowie die Verbesserung alter und die Einführung neuer operativer Techniken kann dazu beitragen, die Mortalität- und Morbidität der Patientinnen zu reduzieren und deren Lebensqualität zu verbessern. 


\section{Summary}

The objective of this study was to retrospectively assess and to describe the outcome of 66 patients, who underwent pelvic exenteration between 1991 und 2005 in the Gynaecological Clinic in Klinikum Kassel. Different clinical and histological factors associated with overall-survival as well as time to relapse were analyzed.

The mean age of patients was 59 years.

Thirty-three anterior, 24 total and nine posterior exenterations were performed. The most patients $(57,6 \%)$ underwent exenterative surgery for cervical cancer. Recurrent malignancy was an indication in 39 patients. Nine patients underwent exenteration for palliation.

Clear histological margins were achieved in 37 patients $(56,1 \%)$. There were lymph node metastases in $45 \%$. Bladder invasion was microscopically detected in $62 \%$, rectal invasion was detected in 20 patients $(30,3 \%)$. Low colorectal anastomosis for fecal diversion was performed in 14\%, urinary diversion included ileal conduit (in 76\%) and continent pouch diversion in $24 \%$.

Forty patients developed postoperative complications; the mortality rate was $6 \%$. The morbidity was higher in the group of patients with a history of irradiation, in the incontinent urinary diversion group and in elderly patients, although no factor reached statistical significance. The 5-year-survival rate was $34 \%$, the mean survival was 22 months. Thirty-seven patients developed recurrence of disease, there was a local recurrence in 25 cases, 12 patients developed distant metastases. The overall-survival was statistically better in patients with negative lymph node status, clear histological margins and in the group of patients undergoing the exenteration for cure.

In summary, pelvic exenteration is an acceptable treatment of locally advanced or recurrent pelvic cancer. The following patients benefit mostly from the pelvic exenteration: patients in good general condition, without prior radiation therapy in the history, 
with negative lymph node status and clear histological margins. Pelvic exenteration for advanced ovarian cancer as well as exenteration for palliation is an option for specific group of patients.

Appropriate patient selection and novel techniques can reduce complications and patient mortality and improve quality of life for patients with advanced pelvic malignancies. 


\section{Literaturverzeichnis}

Aleksic M., Hennes N., Schmitz-Dräger B.J., Ulrich B. (1998). Indikationsstellung und Verlauf bei der totalen pelvinen Exenteration. Chirurg, 69, 450-454.

Andersen B.L. (1985). Sexual functioning morbidity among cancer survivors. Cancer, $55,1835-1842$.

Anthapoulos A.P., Manetta A., Larson J.E. (1989). Pelvic exenteration: a morbidity and mortality analysis of a seven-year experience. Gynecol Oncol, 35, 219-223.

Barber H.R.K. (1988). Pelvic Exenteration. Churchill Livingstone, Edingburgh.

Berek J.S., Howe C., Lagasse L.D., Hacker N.F. (2005). Pelvic exenteration for recurrent gynaecological malignancy: survival and morbidity analysis of the 45-year experience at UCLA. Gynecol Oncol, 99(1), 153-159.

Boyd S.D., Feinberg S.M., Skinner D.G., Lieskovsky G. (1987). Quality of life survey of urinary diversion patients: Comparison of ileal conduits versus continent Kock ileal reservoir. J Urol, 138, 1386-1389.

Bricker E.M. (1950). Bladder substitution after pelvic exenteration. Surg Clin North Am, 30, 1511-21.

Brophy P.F., Hoffman J.P., Eisenberg B.L. (1994). The role of palliative pelvic exenteration. Am J Surg, 167, 386. 
Brunschwig A., Daniel W. (1960). Pelvic Exenteration Operations: With Summary of Sixty-Six Cases Surviving More Than Five Years. Annals of Surgery, 151 (4), 571-576.

Buttarelli M., Houvenaeghel G., Lelievre L., Jacquemier J. (2006). Pelvic posterior exenteration with immediate colo-rectal anastomosis: is it justified and feasible in advanced stage ovarian carcinoma? Ann Chir, 131(8), 431-436.

Crozier M., Morris M., Levenback C., Lucas K.R., Atkinson E.N., Wharton J.T. (1995). Pelvic exenteration for adenocarcinoma of the uterine cervix. Gynecol Oncol, 58(1), 74-78.

De Wilt J.H., van Leeuwen D.H., Logmans A., Verhoef C., Kirkels W.J., Vermaas M., Asink A.C. (2006). Pelvic exenteration for primary and recurrent gynaecological malignancies. Eur J Obstet Gynecol Reprod Biol, 134 (2), 243-248.

Di Saia P.J., Morrow C.P. (1973). Pelvic Exenteration. Calif Med, 118, 13-17.

Deutsche Krebsgesellschaft. Kurzgefasste interdisziplinäre Leitlinien (2002). Diagnose und Therapie maligner Erkrankungen. 3. Auflage. Zuckschwerdt Verlag, München Bern Wien New York.

Durgatosh P., Shuaib Z., Vikas M., Ravi K. (2004). Pelvic exenteration: A perspective from a regional cancer center in India. Indian J Cancer, 41(3), 109-114.

Durst J., Rohen J.W. (1990). Bauchchirurgie mit topographischer Anatomie und gastroenterologischer Onkologie. Schattauer Verlag Stutgart-NewYork. 
Egger H., Hünlich T., Turnwald W., Both A: Pelvic exenteration with complete reconstruction for recurrent gynecologic cancer - experience with 300 patients. European Council of Coloproctology. München 30.10.-2.11.1999.

Fleisch M.C., Pantke P., Beckmann M.W., Schnuerch H.G. (2007). Predictors for longterm survival after interdisciplinary salvage surgery for advanced or recurrent gynaecologic cancers. J Surg Oncol, 95(6). 476-484.

Friedberg V., Petri E. (1986). Pelvine Exenteration (Kommentar). Gynäkologe, 19, 86-87.

Gilchrist R.K., Merricks J.W., Harnlin H.H., Rieger I.T. (1950). Construction of a substitute bladder und urethra. Surg Gynecol Obstet, 90, 752-60.

Gillitzer R., Hampel C., Thüroff J.W. (2007). Pelvine Exenteration wegen gynäkologischer Tumoren. Gynäkologe, 40, 883-890.

Goldberg G.L., Sukumvanich P., Einstein M.H., Smith H.O., Anderson P.S., Fields A.L. (2006). Total pelvic exenteration: the Albert Einstein College of Medicine/Montefiore Medical Center Experience (1987 to 2003). Gynecol Oncol, 101 (2), 261-268.

Hafner G.N., Herrera L., Petrelli N.J. (1992). Morbidity and Mortality After Pelvic Exenteration for Colorectal Adenocarcinoma. Ann Surg, 215(1), 63-67.

Hallböök O., Pahlman L., Krog M., Wexner S.D., Sjödahl R. (1996). Randomized comparison of straight and colonic J Pouch anastomosis after low anterior resection. Ann of Surgery, 224, 58-65. 
Harter P., du Bois A., Hahmann M., Hasenburg A., Burges A., Loibl S., Gropp M. (2006). Recurrent ovarian cancer. The Arbeitsgemeinschaft Gynäkologische Onkologie (AGO) DESKTOP OVAR Trial. Ann Surg Oncol, 13, 1702-1710.

Hatch K.D., Shingelton H.M., Potter M.E. (1988). Low rectal resection and anastomosis at the time of pelvic exenteration. Gynecol Oncol, 32, 262-267.

Hauri D. (2005). Rekonstruktive Urologie: ein historischer Überblick. J Urol Urogynäkol, 12(3), 6-16.

Hautmann R.E., de Petriconi R., Gottfried H.W., Kleinschmidt K., Mattes R., Paiss T. (1999). The ileal neobladder. Complications and functional results in 363 patients after 11 years of follow up. J Urol, 161, 422- 428.

Hautmann R.E., Stenzl A., Studer U.E., Volkmer B.G. (2007). Geänderte Indikation zur Harnableitung. Dtsch Ärztebl, 104, 973-978.

Hawighorst S., Fusshöller C., Franz C., Trautmann K., Schmidt M., Schönefuß G. (2004). Gynäkologische Onkologie: systematische Erfassung der therapieabhängigen Lebensqualität zur Verbesserung der Versorgung - eine prospektive Longitudinalstudie. Geburtsh Frauenheilk, 64, 46-52.

Hawighorst-Knapstein S., Fusshöller C., Franz C., Schönefuß G. (2002). Perioperative Aufklärung und Lebensqualität bei onkologischen Patientinnen. Geburtsh Frauenheilk, 62, Suppl. 1, 5-8.

Hilgers R.-D., Bauer P., Schreiber V. (2003). Einführung in die Medizinische Statistik. Berlin: Springer Verlag. 
Hillemans P., Kürzl R., Sommer H., Röper B. (2004). Zervixkarzinomrezidiv. Manual Zervixkarzinom. Tumorzentrum München und W. Zuckschwerdt Verlag München.

Hinman F. (1936). A critical study of the different principles of surgery which have been used in uretero-intestinal implantation. Tr Am Assoc Genito-Urinary Surgeons, 29, $15-156$.

Hirsch H.A., Käser O., Ikle F.A. (1994). Atlas der gynäkologischen Operationen. 5. Auflage. Thieme, Stuttgart, New York.

Hockel M., Dornhofer N. (2006). Pelvic exenteration for gynaecological tumours: achievements and unanswered questions. Lancet Oncol, 7(10), 837-847.

Hohenfellner R. (1994). Ausgewählte urologische OP-Techniken. 1. Auflage. Thieme, Stuttgart, New York.

Houvenaeghel G., Lelievre L., Gonzague-Casabianca L., Buttarelli M., Moutardier V., Goncalves A., Resbeut M. (2006). Long-term survival after concomitant chemotherapy prior to surgery in advanced cervical carcinoma. Gynecol Oncol, 100 (2), 338-343.

Houvenaeghel G., Moutardier V., Karsenty G., Bladou F., Buttarelli M., Lelong B., Delpero J.R. (2004). Major complications of urinary diversion after pelvic exenteration for gynaecological malignancies: a 23-year mono-institutional experience in 124 patients. Gynecol Oncol, 92(2), 680-683.

Hüsler J., Zimmermann H. (1993). Statistische Prinzipien für medizinische Projekte. Bern: Verlag Hans Huber.

Iborra I., Casanova J.L., Solsona E. (2001). Tolerance of external urinary diversion (Bricker) followed for more than 10 years. Eur Urol, (Suppl 5), 39, 146 (abstract 574). 
Ike H., Shimada H., Yamaguchi S. (2003). Outcome of total pelvic exenteration for primary rectal cancer. Dis Colon Rectum, 46(4), 474-480.

Jakowatz J.G., Porudominsky D., Riihimaki D.U. (1985). Complications of pelvic exenteration. Arch Surg, 120, 1261-1265.

Jimenez R.E., Shoup M., Chen A.M. (2003). Contemporary outcome of total pelvic exenteration in the treatment of colorectal cancer. Dis Colon Rectum, 46 (12), 1619-1625.

Karsenty G., Moutardier V., Lelong B., Guiramand J., Houvenagel G., Delpero J.R., Bladou F. (2005). Long-term follow-up of continent urinary diversion after pelvic exenteration for gynaecological malignancies. Gynecol Oncol, 97(2), 524-528.

Ketcham A.S. (1970). Pelvic exenteration for carcinoma of the uterine cervix. Cancer, 26, 513-521.

Kiselow M., Butcher H.R., Jr., Bricker E.M. (1967). Results of the Radical Surgical Treatment of Advanced Pelvic Cancer: A Fifteen-Year Study. Annals of Surgery, 166(3), 428-434.

Koehler C., Marnitz S., Müller M., Hasenbein K., Schneider A. (2006). Indikation zur primären und sekundären Exenteration bei Patientinnen mit Zervixkarzinom. Geburtsh Frauenheilk, 67, (S1).

Kolbert G., Müller G., Kujath P., Bruch H.P. (1997). Beckenexenteration - Indikation, Radikalität und Follow-up demonstriert an 33 klinischen Fällen. Langebecks Arch Chir, Suppl II, (Kongressbericht 1997). 
Kolles H. (1989). Statistische Auswertungen in der Medizin. München: Jungjohan Verlagsgesellschaft Neckarsulm.

Kraybill W.G., Lopez M.J., Bricker E.M. (1988). Total pelvic exenteration as a therapeutic option in advanced malignant disease of the pelvis. Surg Gynecol Obstet, 166, 259-263.

Lambrou N.C., Pearson J.M., Averette H.E. (2005). Pelvic exenteration of gynaecologic malignancy: indications, and technical and reconstructive considerations. Surg Oncol Clin N Am, 14(2), 289-300.

Lampe B., Egger H., Forner D.M. (2005). Funktionelle Organrekonstruktion in der gynäkologischen Onkologie. Geburtsh Frauenheilk, 65, R2-R15.

Lampe B., Stief C., Egger H., Raab H.R. (2004). Lokal weit fortgeschrittene Tumoren im weiblichen Becken - exenterative Operationen und Rekonstruktion. Deutsches Ärzteblatt, 101(11), 577-584.

Lampe B., Forner D., El-Far A., Meyer A. (2005). Exenteration and Assessment of LocoRegional Resectability. Zentralblatt Gynäkologie, 127, 412-425.

Lawhead R.A. Jr., Clark G.C., Smith D.H. (1989). Pelvic exenteration for recurrent or persistent gynaecological malignancies. A 10-jear review of the Memorial Sloan-Kettering Cancer Center experience (1972-1981). Gynecol Oncol, 33, 279-282.

Lawrence W. Jr., Lopez M.J. (2005). Radical surgery for cancer: a historical perspective. Surg Oncol Clin N Am, 14, 441-446.

Lazorthes F., Chiotasso P., Gamagami R.A., Istvan G., Chevreau P. (1997). Late clinical outcome in a randomized prospective comparison of colonic J pouch and straight coloanal anastomosis. Br J Surg, 84, 1449-1451. 
Lopez M.J., Barrios L. (2005). Evolution of Pelvic Exenteration. Surg Oncol Clin N Am, $14,587-606$.

Lopez M.J., Luna-Perez P. (2004). Composite pelvic exenteration: is it worthwhile? Ann Surg Oncol, 11, 27-33.

Madersbacher S., Schmidt J., Eberle J.M. (2003). Long-term outcome of ileal conduit diversion. J Urol, 169, 985-990.

Magrina J.F., Stanhope C.R. (1997). Pelvic exenteration: supralevator, infralevator, and with vulvectomy. Gynecol Oncol, 64(1), 130-135.

Makkas M. (1910). Zur Behandlung der Blasenektopie - Umwandlung des ausgeschalteten Coecum zur Blase und des Appendix zur Urethra. Zentralblatt Chir, 37, 73-6.

Mantyh C.R., Hull T.L., Fazio V.W. (2001). Coloplasty in low colorectal anastomosis. Dis Colon Rectum, 44, 37-42.

Matthews C.M., Morris M., Burke T.W., Gershenson D.M., Wharton J.T., Rutledge F.N. (1992). Pelvic exenteration in the elderly patient. Obstet Gynecol, 79 (5 ( Pt 1)), 773-7.

Miller D.C., Taub D.A., Dunn R.L., Montie J.E., Wie J.T. (2003). The impact of comorbid disease on cancer control and survival following radical cystectomy. J Urol, 169, 105109.

Morgan P.R., Murdoch J.B., Lopes A., Piura B., Monaghan M.J. (1993). The wallence technique of ureteroileal anastomosis and its use in gynaecologic oncology: a study of 81 cases. Obstet Gynecol, 82, 594-597. 
Morley G.W., Hopkins M.P., Lindenauer S.M., Roberts J.A. (1989). Pelvic exenteration, University of Michigan: 100 patients at 5 years. Obstet Gynecol, 74(6), 934-43.

Mourton S.M., Sonoda Y., Abu-Rustum N.R., Bochner B.H. (2007). Resection of recurrent cervical cancer after total pelvic exenteration. Int J Gynecol Cancer, 17(1), 137-140.

Müller R.P., Seegenschmiedt M.H., Höffken K., Junginger Th., Sauer H. (1999).

Common Toxicity Criteria (CTC): Dokumentation von Nebenwirkungen in der Onkologie. Dtsch Arztebl, 96(8).

Nguyen D.Q., McGregor A.D., Freites O., Carr N.D., Beynon J., El-Sharkawi A.M., Lucas M.G. (2005). Exenterative pelvic surgery - eleven year experience of the Swansea Pelvic Oncology Group. Eur J Surg Oncol, 31(10), 1180-1184.

Novak R. (2002). Ileumconduit („Bricker-Blase“). Rekonstruktive urologische Chirurgie. Pabst Science Publishers. 269-280.

Oliveira Poletto A.H., Lopes A., Carvalho A.L. (2004). Pelvic exenteration and sphinkter preservation: an analysis of 96 cases. J Surg Oncol, 86, 122-127.

Pearlman N.W. (1994). Complications of pelvic exenteration. Surg Oncol Clin N Am, 3, 347-355.

Petri E. (2001). Gynäkologische Urologie. Aspekte der interdisziplinären Diagnostik und Therapie. Georg Thieme Verlag, Stuttgart-New York.

Pinelo S., Petiz A., Domingues C., Lopes C. (2006). Pelvic exenterations for gynaecological cancer--a 10 year institutional review. Acta Med Port, 19(2), 99-104. 
Pontenagel U. (2004). Indikationsstellung und Verlauf bei der totalen pelvinen Exenteration. 121. Kongress der Deutschen Gesellschaft für Chirurgie.

Rauchenwald M. (2001). Harnableitungsformen und deren Konsequenz für die Nachsorge. J Urol Urogynäkol, 8 (Sonderheft 3, 20-21.

Rodriguez-Bigas M.A., Petrelli N.J. (1996). Pelvic Exenteration and its Modifications. Am J Surg, 171, 293-301.

Rowland R.G. (1996). Present experience with the Indiana pouch. W J Urol, 14, 92-98.

Schönefuß G., Hawighorst-Knapstein S., Trautmann K., König Y., Knapstein P.G. (2001). Das Körperleben gynäkologischer Patientinnen vor und nach radikaler Chirurgie. Zentralbl Gynäkol, 123, 23-26.

Schröer A., Fischer D., Lüdders D., Diedrich K., Friedrich M. (2006). Rezidivchirugie beim Ovarialkarzinom. Gynäkologe, 36, 774-779.

Sharma S., Odunsi K., Driscoll D., Lele S. (2005). Pelvic exenterations for gynaecological malignancies: twenty-year experience at Roswell Park Cancer Institute. Int J Gynecol Cancer, 15(3), 475-482.

Shepherd J.H., Crowther M.E. (1986). Complications of gynaecological cancer surgery: a review. Journal of the Royal Society of Medicine, 79, 289-293.

Shoup M., Guillem J.G., Alektiar K.M. (2002). Predictors of survival in recurrent rectal cancer after resection and intraoperative radiotherapy. Dis Colon Rectum, 45(5), 585-592.

Soper J.T., Berchuk A., Creasman W.T. (1989). Pelvic exenteration: factors associated with major surgical morbidity. Gynecol Oncol, 35, 93-98. 
Stanhope C.R., Symmonds R.E. (1985). Palliative exenteration: what, when and why? Am J Obstet Gynecol, 152 (1), 12-16.

Stein R. (1996). Harnableitungen nach vorderer oder totaler Exenteration bei fortgeschrittenen Tumoren des weiblichen Genitale. Geburtsh Frauenheilk, 56, M45-M50.

Stief C.G., Raab R. (2002). Interdisziplinary abdomino-urological surgery for advanced colorectal carcinoma involving the urogenital tract. BJU Int, 89, 496-503.

Tamburini M., Filiberti A., Ventafridda V., De Palo G. (1986). Quality of life and psychological state after radical surgery. J Psychosom Obstet Gynecol, 5, 263-269.

Teran-Porcayo M.A., Zeichner-Gancz I., del-Castillo R.A., Beltran-Ortega A., SolorzaLuna G. (2006). Pelvic exenteration for recurrent or persistent cervical cancer: experience of five years at the National Cancer Institute in Mexico. Med Oncol, 23(2), 219-223.

Übelhör R. (1952). Die Darmblase. Langebecks Arch Chir, 271, 202-210.

Vermaas M., Ferenschild F.T., Verhoef C., Nuyttens J.J., Marinelli A.W., Wiggers T., Kirkels W.J., Eggermont A.M., de Wilt J.H. (2007). Total pelvic exenteration for primary locally advanced and locally recurrent rectal cancer. Eur J Surg Oncol, 33 (4), 452-458.

Wiig J.N., Poulsen J.P., Larsen S. (2002). Total pelvic exenteration with preoperative irradiation for advanced primary and recurrent rectal cancer. Eur J Surg, 168, 42-48.

Willis S., Kasperk R., Braun J., Schumpelick V. (2001). Comparison of colonic J-pouch reconstruction and straight coloanal anastomosis after intersphincteric rectal resection. Arch Surg, 386, 193-199. 
Wydra D., Emerich J., Sawicki S., Ciach K., Marciniak A. (2006). Major complications following exenteration in cases of pelvic malignancy: a 10-yaear experience. World J Gastroenterol, 12 (7), 1115-1119.

Yamada K., Ishizawa T., Niwa K. (2002). Pelvic exenteration and sacral resection for locally advanced primary and recurrent rectal cancer. Dis Colon Rectum, 45, 1078-1084. 


\section{Verzeichnis der akademischen Lehrer}

\section{Meine akademischen Lehrer waren die Damen und Herren}

In Krasnodar:

Adamchik, Avakimyan, Ermoshenko, Galenko-Yaroschevski, Kathanov, Litvinova, Lopunova, Mogilnaya, Remizov

In Moskau:

Baranov, Bazhanov, Bochkov, Boguslavskaya, Dedov, Demidov, Eliseev, Grusha, Hritinin, Kiruschenkov, Krasnov, Kukes, Kuzin, Makatsaria, Makolkin, Maslov , Moroz, Nikonov, Ovchinnikov, Pak, Paltsev, Perelmann, Pitel, Pobedinski, Podzolkov, Pokrovski, Potekaev, Sapin, Shehter, Sirkin, Strizhakov, Stulmann, Svistushkin, Tsoi, Tuvina, Vetshev, Yahno

In Marburg:

Goerke, Schmidt, Schulz

In Kassel:

Dimpfl, Faß, Löser 


\section{Danksagung}

Meinem Doktorvater, Herrn Prof. Dr. Th. Dimpfl möchte ich für die Überlassung des Themas und die außerordentlich freundliche und hilfreiche Betreuung in allen Phasen dieser Doktorarbeit ganz herzlich danken.

Mein besonderer Dank gilt auch Herrn Dr. H. Urbanczyk und Herrn Dr. A. Paseka für die tatkräftige Unterstützung während der Durchführung der Arbeit.

Ich danke meinem Mann Jesco, der stets mit Rat und Tat an meiner Seite stand.

Ganz besonderes bedanke ich mich bei meinen Eltern und meiner Schwester für die großartige Unterstützung, die es mir ermöglicht hat, diesen Weg zu gehen. 